\title{
Null Geodesic Congruences, Asymptotically-Flat Spacetimes and Their Physical Interpretation
}

\author{
Timothy M. Adamo \\ Mathematical Institute, \\ University of Oxford, U.K. \\ email: adamo@maths.ox.ac.uk \\ Ezra T. Newman \\ Department of Physics and Astronomy, \\ University of Pittsburgh, U.S.A. \\ email: newman@pitt.edu \\ Carlos Kozameh \\ Facultad de Matemática, Astronomía y Física, \\ Universidad Nacional de Córdoba, Argentina \\ email: kozameh@famaf.unc.edu.ar
}

Accepted on 16 January 2012

Published on 23 January 2012

\begin{abstract}
A priori, there is nothing very special about shear-free or asymptotically shear-free null geodesic congruences. Surprisingly, however, they turn out to possess a large number of fascinating geometric properties and to be closely related, in the context of general relativity, to a variety of physically significant effects. It is the purpose of this paper to try to fully develop these issues.

This work starts with a detailed exposition of the theory of shear-free and asymptotically shear-free null geodesic congruences, i.e., congruences with shear that vanishes at future conformal null infinity. A major portion of the exposition lies in the analysis of the space of regular shear-free and asymptotically shear-free null geodesic congruences. This analysis leads to the space of complex analytic curves in an auxiliary four-complex dimensional space, $\mathcal{H}$-space. They in turn play a dominant role in the applications.

The applications center around the problem of extracting interior physical properties of an asymptotically-flat spacetime directly from the asymptotic gravitational (and Maxwell) field itself, in analogy with the determination of total charge by an integral over the Maxwell field at infinity or the identification of the interior mass (and its loss) by (Bondi's) integrals of the Weyl tensor, also at infinity.

More specifically, we will see that the asymptotically shear-free congruences lead us to an asymptotic definition of the center-of-mass and its equations of motion. This includes a kinematic meaning, in terms of the center-of-mass motion, for the Bondi three-momentum. In addition, we obtain insights into intrinsic spin and, in general, angular momentum, including an angular-momentum-conservation law with well-defined flux terms. When a Maxwell field is present, the asymptotically shear-free congruences allow us to determine/define at infinity a center-of-charge world line and intrinsic magnetic dipole moment.
\end{abstract}

This review is licensed under a Creative Commons Attribution-Non-Commercial-NoDerivs 3.0 Germany License. http://creativecommons.org/licenses/by-nc-nd/3.0/de/ 


\section{Imprint / Terms of Use}

Living Reviews in Relativity is a peer reviewed open access journal published by the Max Planck Institute for Gravitational Physics, Am Mühlenberg 1, 14476 Potsdam, Germany. ISSN 1433-8351.

This review is licensed under a Creative Commons Attribution-Non-Commercial-NoDerivs 3.0 Germany License: http://creativecommons.org/licenses/by-nc-nd/3.0/de/

Because a Living Reviews article can evolve over time, we recommend to cite the article as follows:

Timothy M. Adamo, Ezra T. Newman and Carlos Kozameh,

"Null Geodesic Congruences, Asymptotically-Flat Spacetimes and Their Physical Interpretation",

Living Rev. Relativity, 15, (2012), 1. [Online Article]: cited [<date>], http://www.livingreviews.org/lrr-2012-1

The date given as $<$ date $>$ then uniquely identifies the version of the article you are referring to. 


\section{Article Revisions}

Living Reviews supports two ways of keeping its articles up-to-date:

Fast-track revision A fast-track revision provides the author with the opportunity to add short notices of current research results, trends and developments, or important publications to the article. A fast-track revision is refereed by the responsible subject editor. If an article has undergone a fast-track revision, a summary of changes will be listed here.

Major update A major update will include substantial changes and additions and is subject to full external refereeing. It is published with a new publication number.

For detailed documentation of an article's evolution, please refer to the history document of the article's online version at http://www. livingreviews.org/lrr-2012-1.

23 January 2012: These revisions were done exclusively by Adamo and Newman, therefore the author order was slightly changed. There was nothing essentially wrong in the earlier version, but we have included several new results (in the text and in appendices), corrected an error of interpretation in Section 7, and (the main reason for the revision) we found much easier ways of doing some of the long calculations with very much simpler arguments. Roughly the changes are: Aside from a few word changes,

1. We have extended the glossary at the beginning by including a Table 2 for the geometric quantities.

2. In Section 3.2 we have added in a fair amount to the discussion of real structures associated with the complex world lines.

3. The major revisions are in Section 6. Here we basically rewrote the entire section; i.e., the derivation of our major results, using much simpler arguments. This greatly shortened the derivation and associated argument.

4. In the Section 7, we corrected an error in the discussion of representation theory.

5. We added items in Section 8 and changed some of the wording in Section 8.1.

6. 19 new references were added.

7. The Acknowledgements 9 were changed.

8. Two new Appendices (E, F) were added presenting new material found since the previous version. 


\section{Contents}

1 Introduction $\quad 7$

1.1 Notation and definitions . . . . . . . . . . . . . . . . . . . . 11

1.2 Glossary of symbols and units . . . . . . . . . . . . . . . . . . 14

2 Foundations $\quad 17$

2.1 Asymptotic flatness and $\mathfrak{I}^{+} \ldots \ldots \ldots \ldots \ldots$. . . . . . . . . . . . . 17

2.2 Bondi coordinates and null tetrad . . . . . . . . . . . . . . . . . 17

2.3 The optical equations . . . . . . . . . . . . . . . . . . . . . . . 20

2.4 The Newman-Penrose formalism . . . . . . . . . . . . . . . . . . . . . . . . . 21

2.5 The Bondi-Metzner-Sachs group . . . . . . . . . . . . . . . . . . 25

2.6 Algebraically-special metrics and the Goldberg-Sachs theorem . . . . . . . . . 25

3 Shear-Free NGCs in Minkowski Space $\quad 27$

3.1 The flat-space good-cut equation and good-cut functions . . . . . . . . . . . . . . 27

3.2 Real cuts from the complex good cuts, I . . . . . . . . . . . . . . . . . . 30

3.3 Approximations . . . . . . . . . . . . . . . . . . . . 32

3.4 Asymptotically-vanishing Maxwell fields . . . . . . . . . . . . . . . . . . . . . . 33

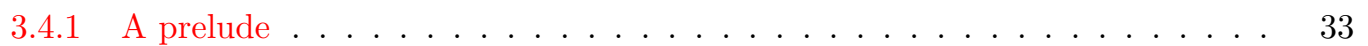

3.4.2 Asymptotically-vanishing Maxwell fields: General properties . . . . . . . . . 34

3.4.3 A coordinate and tetrad system attached to a shear-free NGC . . . . . . . 35

3.4 .4 Complex Liénard-Wiechert Maxwell field . . . . . . . . . . . . . . . . . . 36

3.4.5 Asymptotically vanishing Maxwell fields \& shear-free NGCs . . . . . . . . . 38

3.4.6 The complex center of charge . . . . . . . . . . . . . . . . 40

4 The Good-Cut Equation and $\mathcal{H}$-Space $\quad 41$

4.1 Asymptotically shear-free NGCs and the good-cut equation . . . . . . . . . . . . . 41

$4.2 \mathcal{H}$-space and the good-cut equation . . . . . . . . . . . . . . . . . 43

4.2.1 Solutions to the shear-free equation . . . . . . . . . . . . . . . . . 44

4.3 Real cuts from the complex good cuts, II . . . . . . . . . . . . . . . . . . . . . 45

4.4 Summary of Real Structures . . . . . . . . . . . . . . . . . . . 46

5 Simple Applications $\quad 48$

5.1 Linearized off Schwarzschild . . . . . . . . . . . . . . . . . . . . . . . . . . 48

5.2 The Robinson-Trautman metrics . . . . . . . . . . . . . . . . . . . . . . 50

5.3 Type II twisting metrics . . . . . . . . . . . . . . . . . . . . . . . . . . . 51

5.4 Asymptotically static and stationary spacetimes. . . . . . . . . . . . . . 52

6 Main Results $\quad 54$

6.1 A brief summary - Before continuing . . . . . . . . . . . . . . . . . . . 54

6.2 The complex center-of-mass world line . . . . . . . . . . . . . . . . 57

6.3 The evolution of the complex center of mass . . . . . . . . . . . . . . . . . 59

6.4 The evolution of the Bondi energy-momentum . . . . . . . . . . . . . . . 61

6.5 Other related results . . . . . . . . . . . . . . . . . . . . 63

7 Gauge (BMS) Invariance $\quad 64$ 
8 Discussion/Conclusion $\quad 69$

8.1 History /background . . . . . . . . . . . . . . . . . . . 69

8.2 Other choices for physical identification . . . . . . . . . . . . . . 69

8.3 Predictions ........................... . . . 70

8.4 Summary of results . . . . . . . . . . . . . . . . . . . . . . . . . . . . . . . . . . . . . . . .

8.5 Issues and open questions . . . . . . . . . . . . . . . . . . . . . . . . . . . . . . . . . . . . . . . . . .

8.6 New interpretations and future directions . . . . . . . . . . . . . . 73

9 Acknowledgments $\quad 74$

$\begin{array}{lr}\text { A Twistor Theory } & 75\end{array}$

$\begin{array}{lr}\text { B CR Structures } & \mathbf{7 7}\end{array}$

C Tensorial Spin- $s$ Spherical Harmonics $\quad 79$

C.1 Clebsch-Gordon expansions . . . . . . . . . . . . . . . . . . 81

$\begin{array}{lr}\text { D } \mathcal{H} \text {-Space Metric } & 82\end{array}$

E Shear-Free Congruences from Complex World Lines $\quad 84$

F The Generalized Good-Cut Equation $\quad 85$

$\begin{array}{lr}\text { References } & 88\end{array}$

\section{List of Tables}

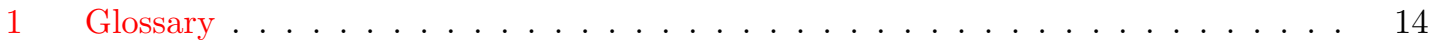

2 Units ............................. 16 



\section{Introduction}

Though from the very earliest days of Lorentzian geometries, families of null geodesics (null geodesic congruences (NGCs)) were obviously known to exist, it nevertheless took many years for their significance to be realized. It was from the seminal work of Bondi [16], with the introduction of null surfaces and their associated null geodesics used for the study of gravitational radiation, that the importance of NGCs became recognized. To analyze the differential structure of such congruences, Sachs [72] introduced the fundamental 'tools', known as the optical parameters, namely, the divergence, the twist (or curl) and the shear of the congruence. From the optical parameters one then could classify congruences by the vanishing (or the asymptotic vanishing) of one or more of these parameters. All the different classes exist in flat space but, in general, only special classes exist in arbitrary spacetimes. For example, in flat space, divergence-free congruences always exist, but for nonflat vacuum spacetimes they exist only in the case of certain high symmetries. On the other hand, twist-free congruences (null surface-forming congruences) exist in all Lorentzian spacetimes. General vacuum spacetimes do not allow shear-free congruences, though all asymptotically-flat spacetimes do allow asymptotically shear-free congruences, a natural generalization of shear-free congruences, to exist.

Our primary topic of study will be the cases of shear-free and asymptotically shear-free NGCs. In flat space the general shear-free congruences have been extensively studied. However, only recently has the special family of regular congruences been investigated. In general, as mentioned above, vacuum (or Einstein-Maxwell) metrics do not possess shear-free congruences; the exceptions being the algebraically-special metrics, all of which contain one or two such congruences. On the other hand, all asymptotically-flat spacetimes possess large numbers of regular asymptotically shear-free congruences. By a 'regular congruence' we mean a NGC that has all of its null geodesics coming from the interior of the spacetime and intersecting with future null infinity; none of its geodesics lie on future null infinity. This condition on the congruences play a fundamental role in the present work.

A priori there does not appear to be anything very special about shear-free or asymptotically shear-free NGCs. However, over the years, simply by observing a variety of topics, such as the classification of Maxwell and gravitational fields (algebraically-special metrics), twistor theory, $\mathcal{H}$-space theory and asymptotically-flat spacetimes, there have been more and more reasons to consider them to be of considerable importance. One of the earliest examples of this is Robinson's [70] demonstration that a necessary condition for a curved spacetime to admit a null solution of Maxwell's equation is that there be, in that space, a congruence of null, shear-free geodesics. Recent results have shown that the regular congruences - both the shear-free and the asymptotically shear-free congruences - have certain very attractive and surprising properties; each congruence is determined by a complex analytic curve in the auxiliary complex space that is referred to as $\mathcal{H}$-space. For asymptotically-flat spacetimes, some of these curves contain a great deal of physical information about the spacetime itself [42, 40, 41].

It is the main purpose of this work to give a relatively complete discussion of these issues. However, to do so requires a digression.

A major research topic in general relativity (GR) for many years has been the study of asymptotically-flat spacetimes. Originally, the term 'asymptotically flat' was associated with gravitational fields, arising from finite bounded sources, where infinity was approached along spacelike directions (e.g., [11, 74]). Then the very beautiful work of Bondi [16] showed that a richer and more meaningful idea to be associated with 'asymptotically flat' was to study gravitational fields in which infinity was approached along null directions. This led to an understanding of gravitational radiation via the Bondi energy-momentum loss theorem, one of the profound results in GR. The Bondi energy-momentum loss theorem, in turn, was the catalyst for the entire contemporary subject of gravitational radiation and gravitational wave detectors. The fuzzy idea of where and 
what is infinity was clarified and made more specific by the work of Penrose [62, 63] with the introduction of the conformal compactification (via the rescaling of the metric) of spacetime, whereby infinity was added as a boundary and brought into a finite spacetime region. Penrose's infinity or spacetime boundary, referred to as Scri or $\mathfrak{I}$, has many sub-regions: future null infinity, $\mathfrak{I}^{+}$; past null infinity, $\mathfrak{I}^{-}$; future and past timelike infinity, $\boldsymbol{I}^{+}$and $\boldsymbol{I}^{-}$; and spacelike infinity, $\boldsymbol{I}^{0}$ [23]. In the present work, $\mathfrak{I}^{+}$and its neighborhood will be our primary arena for study.

A basic question for us is what information about the interior of the spacetime can be obtained from a study of the asymptotic gravitational field; that is, what can be learned from the remnant of the full field that now 'lives' or is determined on $\mathfrak{I}^{+}$? This quest is analogous to obtaining the total interior electric charge or the electromagnetic multipole moments directly from the asymptotic Maxwell field, i.e., the Maxwell field at $\mathfrak{I}^{+}$, or the Bondi energy-momentum four-vector from the gravitational field (Weyl tensor) at $\mathfrak{I}^{+}$. However, the ideas described and developed here are not really in the mainstream of GR; they may lie outside the usual interest and knowledge of many researchers. Nevertheless, they are strictly within GR: no new physics is introduced; only the vacuum Einstein or Einstein-Maxwell equations are used. The ideas come simply from observing (discovering) certain unusual and previously overlooked features of solutions to the Einstein equations and their asymptotic behavior.

These observations, as mentioned earlier, centered on the realization of the remarkable properties and importance of the special families of null geodesics: the regular shear-free and asymptotically shear-free NGCs.

The most crucial and striking of these overlooked features (mentioned now but fully developed later) are the following: in flat space every regular shear-free NGC is determined by the arbitrary choice of a complex analytic world line in complex Minkowski space, $\mathbb{M}_{\mathbb{C}}$. Furthermore and more surprising, for every asymptotically-flat spacetime, every regular asymptotically shear-free NGC is determined by the given Bondi shear (given for the spacetime itself) and by the choice of an arbitrary complex analytic world line in an auxiliary complex four-dimensional space, $\mathcal{H}$-space, endowed with a complex Ricci-flat metric. In other words, the space of regular shear-free and asymptotically shear-free NGCs are both determined by arbitrary analytic curves in $\mathbb{M}_{\mathbb{C}}$ and $\mathcal{H}$ space respectively [42, 40, 39].

Eventually, a unique complex world line in this space is singled out, with both the real and imaginary parts being given physical meaning. The detailed explanation for the determination of this world line is technical and reserved for a later discussion. However, a rough intuitive idea can be given in the following manner.

The idea is a generalization of the trivial procedure in electrostatics of first defining the electric dipole moment, relative to an origin, and then shifting the origin so that the dipole moment vanishes and thus obtaining the center of charge. Instead, we define, on $\mathfrak{I}^{+}$, with specific Bondi coordinates and tetrad, the complex mass dipole moment (the real mass dipole plus ' $i$ ' times angular momentum) from certain components of the asymptotic Weyl tensor. (The choice of the specific Bondi system is the analogue of the choice of origin in the electrostatic case.) Then, knowing how the asymptotic Weyl tensor transforms under a change of tetrad and coordinates, one sees how the complex mass dipole moment changes when the tetrad is rotated to one defined from the asymptotically shear-free congruence. By setting the transformed complex mass dipole moment to zero, the unique complex world line, identified as the complex center of mass, is obtained. A similar process can be used in Einstein-Maxwell theory to obtain a complex center of charge.

This procedure, certainly unusual and perhaps appearing ambiguous, does logically hold together. The real justification for these identifications comes not from this logical structure though, but rather from the observed equivalence of the derived results from these identifications with well-known classical mechanical and electrodynamical relations. These derived results involve both kinematical and dynamical relations. Though they will be discussed at length later, we mention that they range from a kinematic expression for the Bondi momentum of the form, $P=M v+\ldots$; 
a derivation of Newton's second law, $F=M a$; and a conservation law for angular momentum with a well-known angular momentum flux, to the prediction of the Dirac value of the gyromagnetic ratio. We note that, for the charged spinning particle metric [53], the imaginary part of the world line is indeed the spin angular momentum, a special case of our results.

A major early clue that shear-free NGCs were important in GR was the discovery of the (vacuum or Einstein-Maxwell) algebraically special metrics. These metrics are defined by the algebraic degeneracy in their principle null vectors, which form (by the Goldberg-Sachs theorem [29]) a null congruence which is both geodesic and shear-free. For the asymptotically-flat algebraicallyspecial metrics, this shear-free congruence (a very special congruence from the set of asymptotically shear-free congruences) determines a unique world line in the associated auxiliary complex $\mathcal{H}$-space. This shear-free congruence (with its associated complex world line) is a special case of the above argument of transforming to the complex center of mass. Our general asymptotically-flat situation is, thus, a generalization of the algebraically-special case. Much of the analysis leading to the transformation of the complex dipoles in the case of the general asymptotically-flat spaces arose from generalizing the case of the algebraically-special metrics.

To get a rough feeling (first in flat space) of how the curves in $\mathbb{M}_{\mathbb{C}}$ are connected with the shear-free congruences, we first point out that the shear-free congruences are split into two classes: the twisting congruences and the twist-free ones. The regular twist-free ones are simply the null geodesics (the generators) of the light cones with apex on an arbitrary timelike Minkowski space world line. Observing backwards along these geodesics from afar, one 'sees' the world line. The regular twisting congruences are generated in the following manner: consider the complexification of Minkowski space, $\mathbb{M}_{\mathbb{C}}$. Choose an arbitrary complex (analytic) world line in $\mathbb{M}_{\mathbb{C}}$ and construct its family of complex light cones. The projection into the real Minkowski space, $\mathbb{M}$, of the complex geodesics (the generators of these complex cones), yields the real shear-free twisting NGCs [7]. The twist contains or 'remembers' the apex on the complex world line, and looking backwards via these geodesics, one appears 'to see' the complex world line. In the case of asymptotically shear-free congruences in curved spacetime, one cannot trace the geodesics back to a complex world line. However, one can have the illusion (i.e., a virtual image) that the congruence is coming from a complex world line. It is from this property that we can refer to the asymptotically shearfree congruences as lying on generalized light cones. There is a duality between the real twisting congruences and the complex congruences coming from the complex world line: knowledge of one determines the other.

The analysis of the geometry of the asymptotically shear-free NGCs is greatly facilitated by the introduction of Good-Cut Functions (GCFs). Each GCF is a complex slicing of $\mathfrak{I}^{+}$from which the associated asymptotically shear-free NGC and world line can be easily obtained. For the special world line and congruence that leads to the complex center of mass, there is a unique GCF that is referred to as the Universal-Cut Function (UCF).

Information about a variety of objects is contained in and can be easily calculated from the UCF: the unique complex world line; the direction of each geodesic of the congruence arriving at $\mathfrak{I}^{+}$; and the Bondi asymptotic shear of the spacetime. The ideas behind the GCFs and UCF are due to some very pretty mathematics arising from the study of the 'good-cut equation' and its complex four-dimensional solution space, $\mathcal{H}$-space [49, 37]. In flat space almost every asymptotically vanishing Maxwell field determines its own Universal Cut Function, where the associated world line determines both the center of charge and the magnetic dipole moment. In general, for Einstein-Maxwell fields, there will be two different UCFs, (and hence two different world lines), one for the Maxwell field and one for the gravitational field. The physically interesting special case where the two world lines coincide will be discussed.

In this work, we seek to provide a comprehensive overview of the theory of asymptotically shearfree NGCs, as well as their physical applications to both flat and asymptotically-flat spacetimes. The resulting theoretical framework unites ideas from many areas of relativistic physics and has 
a crossover with several areas of mathematics, which had previously appeared short of physical applications.

The main mathematical tool used in our description of $\mathfrak{I}^{+}$is the Newman-Penrose (NP), or Spin-Coefficient (SC), formalism [55]. Spherical functions are expanded in spin- $s$ tensor harmonics [59]; in our approximations only the $l=0,1,2$ harmonics are retained. Basically, the detailed calculations should be considered as expansions around the Reissner-Nordström metric, which is treated as zeroth order; all other terms being small, i.e., at least first order. We retain terms only to second order.

In Section 2, we give a brief review of Penrose's conformal null infinity $\mathfrak{I}$ along with an exposition of the NP formalism and its application to Maxwell theory and asymptotically-flat spacetimes. There is then a description of $\mathfrak{I}^{+}$, the stage on which most of our calculations take place. The Bondi mass aspect (a function on $\mathfrak{I}^{+}$) is defined by the asymptotic Weyl tensor and asymptotic shear; from it we obtain the physical identifications of the Bondi mass and linear momentum. Also discussed is the asymptotic symmetry group of $\mathfrak{I}^{+}$, the Bondi-Metzner-Sachs (BMS) group [16, 72, 56, 65]. The Bondi mass and linear momentum become basic for the physical identification of the complex center-of-mass world line.

Section 3 contains the detailed analysis of shear-free NGCs in Minkowski spacetime. This includes the identification of the flat space GCFs from which all regular shear-free congruences can be found. We also show the intimate connection between the flat space GCFs, the (homogeneous) good-cut equation, and $\mathbb{M}_{\mathbb{C}}$. As applications, we investigate the UCF associated with asymptotically-vanishing Maxwell fields and in particular the shear-free congruences associated with the Liénard-Wiechert (and complex Liénard-Wiechert) fields. This allows us to identify a real (and complex) center-of-charge world line, as mentioned earlier.

In Section 4, we give an overview of the machinery necessary to deal with twisting asymptotically shear-free NGCs in asymptotically-flat spacetimes. This involves a discussion of the theory of $\mathcal{H}$-space, the construction of the good-cut equation from the asymptotic Bondi shear and its complex four-parameter family of solutions. We point out how the simple Minkowski space of the preceding Section 3 can be seen as a special case of the more general theory outlined here. These results have ties to Penrose's twistor theory and the theory of Cauchy-Riemann (CR) structures; an explanation of these crossovers is given in Appendices A and B.

Section 5 provides some examples of these ideas in action. We discuss linear perturbations off the Schwarzschild metric, Robinson-Trautman and twisting type II algebraically special metrics, as well as asymptotically stationary spacetimes, and illustrate how the good-cut equation can be solved and the UCF determined (explicitly or implicitly) in each case.

In Section 6, the methodology laid out in the previous Sections 3, 4 and 5 is applied to the general class of asymptotically-flat spacetimes: vacuum and Einstein-Maxwell. Here, reviewing the material of the previous section, we use the solutions of the good-cut equation to determine all regular asymptotically shear-free NGCs by first choosing arbitrary world lines in the solution space and then singling out a unique one which determines the UCF (two world lines exist in the Einstein-Maxwell case, one for the gravitational field, the other for the Maxwell field). This identification of the unique lines comes from a study of the transformation properties, at $\mathfrak{I}^{+}$, of the asymptotically-defined mass and spin dipoles and the electric and magnetic dipoles. The work of Bondi, with the identification of energy-momentum and its evolution, allows us to make a series of surprising further physical identifications and predictions. In addition, with a slightly different approximation scheme, we discuss our ideas applied to the asymptotic gravitational field with an electromagnetic dipole field as the source.

Section 7 contains an analysis of the gauge (or BMS) invariance of our results.

Section 8, the Discussion/Conclusion section, begins with a brief history of the origin of the ideas developed here, followed by comments on alternative approaches, possible physical predictions from our results, a summary and open questions. 
Finally, we conclude with six appendices, which contain several mathematical crossovers that were frequently used or referred to in the text: twistor theory (A); CR structures (B); a brief exposition of the tensorial spherical harmonics [59] and their Clebsch-Gordon product decompositions (C); an overview of the metric construction on $\mathcal{H}$-space (D); the description of certain real aspects of complex Minkowski space world lines (E); and a discussion of the 'generalized good-cut equation' with an arbitrary conformal factor $(\mathrm{F})$.

\subsection{Notation and definitions}

The following contains the notational conventions that will be in use throughout the course of this review.

- We use the symbols ' $l$ ', ' $m$ ', ' $n$ ' ... with several different 'decorations' but always meaning a null tetrad or a null tetrad field.

a) Though in places, e.g., in Section 2.4, the symbols, $l^{a}, m^{a}, n^{a} \ldots$, i.e., with an $a, b, c \ldots$ can be thought of as the abstract representation of a null tetrad (i.e., Penrose's abstract index notation [66]), in general, our intention is to describe vectors in a coordinate representation.

b) The symbols, $l^{a}, l^{\# a}, l^{* a}$ most often represent the coordinate versions of different null geodesic tangent fields, e.g., one-leg of a Bondi tetrad field or some rotated version.

c) The symbol, $\hat{l}^{a}$, (with hat) has a very different meaning from the others. It is used to represent the Minkowski components of a normalized null vector giving the null directions on an arbitrary light cone:

$$
\hat{l}^{a}=\frac{\sqrt{2}}{2(1+\zeta \bar{\zeta})}(1+\zeta \bar{\zeta}, \zeta+\bar{\zeta}, i \bar{\zeta}-i \zeta,-1+\zeta \bar{\zeta}) \equiv\left(\frac{\sqrt{2}}{2} Y_{0}^{0}, \frac{1}{2} Y_{1 i}^{0}\right) .
$$

As the complex stereographic coordinates $(\zeta, \bar{\zeta})$ sweep out the sphere, the $\hat{l}^{a}$ sweeps out the entire set of directions on the future null cone. The other members of the associated null tetrad are

$$
\begin{aligned}
\hat{m}^{a} & =\frac{\sqrt{2}}{2(1+\zeta \bar{\zeta})}\left(0,1-\bar{\zeta}^{2},-i\left(1+\bar{\zeta}^{2}\right), 2 \bar{\zeta}\right), \\
\hat{n}^{a} & =\frac{\sqrt{2}}{2(1+\zeta \bar{\zeta})}(1+\zeta \bar{\zeta},-(\zeta+\bar{\zeta}), i \zeta-i \bar{\zeta}, 1-\zeta \bar{\zeta}) .
\end{aligned}
$$

- Several different time variables $\left(u_{\mathrm{B}}, u_{\mathrm{ret}}, \tau, s\right)$ and derivatives with respect to them are used. The Bondi time, $u_{\mathrm{B}}$, is closely related to the retarded time, $u_{\text {ret }}=\sqrt{2} u_{\mathrm{B}}$. The use of the retarded time, $u_{\text {ret }}$, is important in order to obtain the correct numerical factors in the expressions for the final physical results. Derivatives with respect to these variables are represented by

$$
\begin{aligned}
\partial_{u_{\mathrm{B}}} K & \equiv \dot{K}, \\
\partial_{u_{\mathrm{ret}}} K & \equiv K^{\prime}=\frac{\sqrt{2}}{2} \dot{K} .
\end{aligned}
$$

The $u_{\text {ret }}, \tau, s$, derivatives are denoted by the same prime $\left({ }^{\prime}\right)$ since it is always applied to functions with the same functional argument. Though we are interested in real physical spacetime, often the time variables $\left(u_{\mathrm{ret}}, u_{\mathrm{B}}, \tau\right)$ take complex values close to the real $(s$ is always real). Rather than putting on 'decorations' to indicate when they are real or complex 
(which burdens the expressions with an overabundance of different symbols), we leave reality decisions to be understood from context. In a few places where the reality of the particular variable is manifestly first introduced (and is basic) we decorate the symbol by a superscript $(R)$, i.e., $u_{\mathrm{B}}^{(R)}$ or $u_{\text {ret }}^{(R)}$. After their introduction we revert to the undecorated symbol.

Remark 1. At this point we are taking the velocity of light as $c=1$ and omitting it; later, when we want the correct units to appear explicitly, we restore the $c$. This entails, via $\tau \rightarrow c \tau$, $s \rightarrow c s$, changing the prime derivatives to include the $c$, i.e.,

$$
K^{\prime} \rightarrow c^{-1} K^{\prime} .
$$

- Often the angular (or sphere) derivatives, $\check{\partial}$ and $\bar{\partial}$, are used. The notation $\check{\partial}_{(\alpha)} K$ means, apply the $\partial$ operator to the function $K$ while holding the variable $(\alpha)$ constant.

- The complex conjugate is represented by the overbar, e.g., $\bar{\zeta}$. When a complex variable, $\tilde{\zeta}$, is close to the complex conjugate of $\zeta$, but independent, we use $\tilde{\zeta} \approx \bar{\zeta}$.

Frequently, in this work, we use terms that are not in standard use. It seems useful for clarity to have some of these terms defined from the outset:

- As mentioned earlier, we use the term 'generalized light cones' to mean (real) NGCs that appear to have their apexes on a world line in the complexification of the spacetime. A detailed discussion of this will be given in Sections 3 and 4 .

- The term 'complex center of mass' (or 'complex center of charge') is frequently used. Up to the choice of constants (to give correct units) they basically lead to the 'mass-dipole plus " $i$ " angular momentum' (or 'real electric-dipole plus " $i$ " magnetic dipole moment'). There will be two different types of these 'complex centers of ...'; one will be geometrically defined or intrinsic, i.e., independent of the choice of coordinate system, the other will be relative, i.e., it will depend on the choice of (Bondi) coordinates. The relations between them are nonlinear and nonlocal.

- A very important technical tool used throughout this work is a class of complex analytic functions, $u_{\mathrm{B}}=G(\tau, \zeta, \bar{\zeta})$, referred to as Good-Cut Functions, (GCFs) that are closely associated with shear-free NGCs. The details are given later. For any given asymptotically-vanishing Maxwell field with nonvanishing total charge, the Maxwell field itself allows one, on physical grounds, to choose a unique member of the class referred to as the (Maxwell) UniversalCut Function (UCF). For vacuum asymptotically-flat spacetimes, the Weyl tensor allows the choice of a unique member of the class referred to as the (gravitational) UCF. For EinsteinMaxwell there will be two such functions, though in important cases they will coincide and be referred to as UCFs. When there is no ambiguity, in either case, they will simple be UCFs.

- A notational irritant arises from the following situation. Very often we expand functions on the sphere in spin-s harmonics, as, e.g.,

$$
\chi=\chi^{0} Y_{0}+\chi^{i} Y_{1 i}(\zeta, \bar{\zeta})+\chi^{i j} Y_{2 i j}(\zeta, \bar{\zeta})+\chi^{i j k} Y_{3 i j k}(\zeta, \bar{\zeta})+\ldots,
$$

where the indices, $i, j, k \ldots$ represent three-dimensional Euclidean indices. To avoid extra notation and symbols we write scalar products and cross-products without the use of an explicit Euclidean metric, leading to awkward expressions like

$$
\begin{aligned}
\vec{\eta} \cdot \vec{\lambda} & \equiv \eta^{i} \lambda^{i} \equiv \eta^{i} \lambda_{i}, \\
\mu^{k} & =(\vec{\eta} \times \vec{\lambda})^{k} \equiv \eta^{i} \lambda^{j} \epsilon_{i j k} .
\end{aligned}
$$


This, though easy to understand and keep track of, does run into the unpleasant fact that often the four-vector,

$$
\chi^{a}=\left(\chi^{0}, \chi^{i}\right),
$$

appears as the $l=0,1$ harmonics in the harmonic expansions. Thus, care must be used when lowering or raising the relativistic index, i.e., $\eta_{a b} \chi^{a}=\chi_{b}=\left(\chi^{0},-\chi^{i}\right)$.

- Throughout this review (and especially in Section 6), we will invoke comparisons between our results and those of classical electromagnetism and relativity (cf. [43]). This process rests upon our identifications of the electric and magnetic dipole and quadrupole moments in the spherical harmonic expansions of the Maxwell tensor in the Newman-Penrose formalism. Although the identifications we make are the most natural in our framework, a numerical re-scaling is required to obtain the physical formulae in some cases: in terms of the complex dipole and quadrupole moments used for the electromagnetic field, this is given by

$$
D_{\mathbb{C}}^{i}=D_{\mathbb{C}}^{i \text { physical }}, \quad Q_{\mathbb{C}}^{i j}=\frac{\sqrt{2}}{4} Q_{\mathbb{C}}^{i j \text { physical }} .
$$

The conventions used here were chosen so that the numerical coefficient of $Q_{\mathbb{C}}^{i j}$ in $\phi_{0}^{0}$ was equal to one; this re-scaling can simply be viewed as choosing a different (perhaps less natural) identification for the electromagnetic quadrupole moment, or as a sort of gauge choice for our results. 


\subsection{Glossary of symbols and units}

In this work we make use of substantial notational machinery. The most frequently used symbols and acronyms are gathered here for easy reference:

Table 1: Glossary

\begin{tabular}{|c|c|}
\hline Symbol/Acronym & Definition \\
\hline $\mathfrak{I}^{+}, \mathfrak{I}_{\mathbb{C}}^{+}$ & Future null infinity, Complex future null infinity \\
\hline $\boldsymbol{I}^{+}, \boldsymbol{I}^{-}, \boldsymbol{I}^{0}$ & Future, Past timelike infinity, Spacelike infinity \\
\hline $\mathbb{M}, \mathbb{M}_{\mathbb{C}}$ & Minkowski space, Complex Minkowski space \\
\hline$u_{\mathrm{B}}, u_{\mathrm{ret}}$ & Bondi time coordinate, Retarded Bondi time $\left(\sqrt{2} u_{\mathrm{B}}=u_{\mathrm{ret}}\right)$ \\
\hline$\partial_{u_{\mathrm{B}}} f=\dot{f}$ & Derivation with respect to $u_{\mathrm{B}}$ \\
\hline$\partial_{u_{\mathrm{ret}}} f=f^{\prime}$ & Derivation with respect to $u_{\text {ret }}$ \\
\hline$r$ & Affine parameter along null geodesics \\
\hline$(\zeta, \bar{\zeta})$ & $\left(e^{i \phi} \cot (\theta / 2), e^{-i \phi} \cot (\theta / 2)\right) ;$ stereographic coordinates on $S^{2}$ \\
\hline$Y_{l i \ldots j}^{s}(\zeta, \bar{\zeta})$ & Tensorial spin- $s$ spherical harmonics \\
\hline ஓ, $\bar{\partial}$ & $\begin{array}{l}P^{1-s} \frac{\partial}{\partial \zeta} P^{s}, \quad P^{1+s} \frac{\partial}{\partial \zeta} P^{-s} ; \text { spin-weighted operator on the two- } \\
\text { sphere }\end{array}$ \\
\hline$P$ & Metric function on $S^{2} ;$ often $P=P_{0} \equiv 1+\zeta \bar{\zeta}$ \\
\hline$\partial_{(\alpha)} f$ & $\begin{array}{l}\text { Application of } \partial \text {-operator to } f \text { while the variable } \alpha \text { is held } \\
\text { constant }\end{array}$ \\
\hline$\left\{l^{a}, n^{a}, m^{a}, \bar{m}^{a}\right\}$ & Null tetrad system; $l^{a} n_{a}=-m^{a} \bar{m}_{a}=1$ \\
\hline NGC & Null Geodesic Congruence \\
\hline $\mathrm{NP} / \mathrm{SC}$ & Newman-Penrose/Spin-Coefficient Formalism \\
\hline$\left\{U, X^{A}, \omega, \xi^{A}\right\}$ & Metric coefficients in the Newman-Penrose formalism \\
\hline$\left\{\psi_{0}, \psi_{1}, \psi_{2}, \psi_{3}, \psi_{4}\right\}$ & Weyl tensor components in the Newman-Penrose formalism \\
\hline$\left\{\phi_{0}, \phi_{1}, \phi_{2}\right\}$ & $\begin{array}{l}\text { Maxwell tensor components in the Newman-Penrose formal- } \\
\text { ism }\end{array}$ \\
\hline$\rho$ & Complex divergence of a null geodesic congruence \\
\hline$\Sigma$ & Twist of a null geodesic congruence \\
\hline$\sigma, \sigma^{0}$ & Complex shear, Asymptotic complex shear of a NGC \\
\hline$k$ & $\frac{2 G}{c^{4}} ;$ Gravitational constant \\
\hline$\tau=s+i \lambda=T(u, \zeta, \bar{\zeta})$ & Complex auxiliary $(\mathrm{CR})$ potential function \\
\hline$\partial_{\tau} f=f^{\prime}$ & Derivation with respect to $\tau$ \\
\hline$\check{\partial}_{(\tau)}^{2} G(\tau, \zeta, \bar{\zeta})=\sigma^{0}(G, \zeta, \bar{\zeta})$ & $\begin{array}{l}\text { Good-Cut Equation, describing asymptotically shear-free } \\
\text { NGCs }\end{array}$ \\
\hline
\end{tabular}


Table 1 - Continued

\begin{tabular}{|c|c|}
\hline Symbol/Acronym & Definition \\
\hline$u_{\mathrm{B}}=G(\tau, \zeta, \bar{\zeta})$ & Good-Cut Function $(\mathrm{GCF})$ on $\mathfrak{I}^{+}$ \\
\hline$L\left(u_{\mathrm{B}}, \zeta, \bar{\zeta}\right)=\partial_{(\tau)} G$ & $\begin{array}{l}\text { Stereographic angle field for an asymptotically shear-free NGC } \\
\text { at } \mathfrak{I}^{+}\end{array}$ \\
\hline$\check{\partial}_{\left(u_{\mathrm{B}}\right)} T+L \dot{T}=0$ & CR equation, describing the embedding of $\mathfrak{I}^{+}$into $\mathbb{C}^{2}$ \\
\hline $\mathcal{H}$-space & $\begin{array}{l}\text { Complex four-dimensional solution space to the Good-Cut } \\
\text { Equation }\end{array}$ \\
\hline$D_{\mathbb{C}}^{i}=D_{E}^{i}+i D_{M}^{i}=\frac{1}{2} \phi_{0}^{0 i}$ & Complex electromagnetic dipole \\
\hline$\eta^{a}\left(u_{\mathrm{ret}}\right)$ & Complex center-of-charge world line, lives in $\mathcal{H}$-space \\
\hline$Q_{\mathbb{C}}^{i j}=Q_{E}^{i j}+i Q_{M}^{i j}=\frac{\sqrt{2}}{4} Q_{\mathbb{C}}^{i j \text { physical }}$ & Complex electromagnetic quadrupole \\
\hline$\xi^{a}\left(u_{\mathrm{ret}}\right)$ & Complex center of mass world line, lives in $\mathcal{H}$-space \\
\hline \multicolumn{2}{|l|}{$D_{(\text {grav })}^{i}=D_{(\text {mass })}^{i}+i c^{-1} J^{i}$} \\
\hline$=-\frac{c^{2}}{6 \sqrt{2} G} \psi_{1}^{0 i}$ & Complex gravitational dipole \\
\hline$Q_{\mathrm{Grav}}^{i j}=Q_{\mathrm{Mass}}^{i j}+i Q_{\mathrm{Spin}}^{i j}$ & Complex gravitational quadrupole \\
\hline$u_{\mathrm{B}}=X(\tau, \zeta, \bar{\zeta})$ & $\begin{array}{l}\text { Universal Cut Function (UCF) corresponding to the complex } \\
\text { center of mass world line }\end{array}$ \\
\hline$\xi^{i j}=\frac{\sqrt{2} G}{24 c^{4}} Q_{\text {Grav }}^{i j \prime \prime}$ & $\begin{array}{l}\text { Identification between } l=2 \text { coefficient of the UCF and gravi- } \\
\text { tational quadrupole }\end{array}$ \\
\hline$\Psi \equiv \psi_{2}^{0}+\partial^{2} \overline{\sigma^{0}}+\sigma^{0} \dot{\overline{\sigma^{0}}}=\bar{\Psi}$ & Bondi Mass Aspect \\
\hline$M_{\mathrm{B}}=-\frac{c^{2}}{2 \sqrt{2} G} \Psi^{0}$ & Bondi mass \\
\hline$P^{i}=-\frac{c^{3}}{6 G} \Psi^{i}$ & Bondi linear three-momentum \\
\hline$J^{i}=-\frac{\sqrt{2} c^{3}}{12 G} \operatorname{Im}\left(\psi_{1}^{0 i}\right)$ & Vacuum linear theory identification of angular momentum \\
\hline
\end{tabular}


In much of what follows, we use simplified units where $c=1$. However, in Section 6 we will revert to a notation which makes dependence upon numerical constants explicit for the sake of comparing our results with well-known quantities in classical mechanics and electromagnetism. We therefore include the following reference table for the units of several prominent objects in our calculations to ease in verifying that correct powers of dimensional constants (e.g., $c, G$ ) appear in our final results. Here [.] stands for the units of a given quantity, and

$$
\mathrm{L}=[\text { length }], \quad \mathrm{M}=[\text { mass }], \quad \mathrm{T}=[\text { time }] .
$$

Table 2: Units

\begin{tabular}{ll}
\hline Quantity & Units \\
\hline$[G]$ & $\mathrm{L}^{3} \mathrm{M}^{-1} \mathrm{~T}^{-2}$ \\
{$[q]$} & $\mathrm{M}^{\frac{1}{2}} \mathrm{~L}^{\frac{3}{2}} \mathrm{~T}^{-1}$ \\
{$[k]=\left[G c^{-4}\right]$} & $\mathrm{M}^{-1} \mathrm{~L}^{-1} \mathrm{~T}^{2}$ \\
{$[c \tau]=\left[c u_{\mathrm{B}}\right]=[G(\tau, \zeta, \bar{\zeta})]$} & $\mathrm{L}$ \\
{$\left[\xi^{i}(\tau)\right]=\left[\eta^{i}(\tau)\right]=\left[\xi^{i j}(\tau)\right]$} & $\mathrm{L}$ \\
{$\left[D_{\mathbb{C}}^{i}\right]$} & $\mathrm{M}^{\frac{1}{2}} \mathrm{~L}^{\frac{5}{2}} \mathrm{~T}^{-1}$ \\
{$\left[D_{\text {(grav) }}^{i}\right]$} & $\mathrm{ML}^{i j}$ \\
{$\left[Q_{\mathbb{C}}^{i j}\right]$} & $\mathrm{M}^{\frac{1}{2}} \mathrm{~L}^{\frac{7}{2}} \mathrm{~T}^{-1}$ \\
{$\left[Q_{\text {Grav }}^{i j}\right]$} & $\mathrm{ML}^{2}$ \\
{$\left[J^{i}\right]$} & $\mathrm{ML}^{2} \mathrm{~T}^{-1}$ \\
{$\left[\phi_{0}\right]=\left[\phi_{1}\right]=\left[\phi_{2}\right]$} & $\mathrm{M}^{\frac{1}{2}} \mathrm{~L}^{-\frac{1}{2}} \mathrm{~T}^{-1}$ \\
{$\left[\phi_{0}^{0}\right]$} & $\mathrm{M}^{\frac{1}{2}} \mathrm{~L}^{\frac{5}{2}} \mathrm{~T}^{-1}$ \\
{$\left[\phi_{1}^{0}\right]$} & $\mathrm{M}^{\frac{1}{2}} \mathrm{~L}^{\frac{3}{2}} \mathrm{~T}^{-1}$ \\
{$\left[\phi_{2}^{0}\right]$} & $\mathrm{M}^{\frac{1}{2}} \mathrm{~L}^{\frac{1}{2}} \mathrm{~T}^{-1}$ \\
{$\left[\psi_{0}\right]=\left[\psi_{1}\right]=\left[\psi_{2}\right]=\left[\psi_{3}\right]=\left[\psi_{4}\right]$} & $\mathrm{L}^{-2}$ \\
{$\left[\psi_{0}^{0}\right]$} & $\mathrm{L}^{3}$ \\
{$\left[\psi_{1}^{0}\right]$} & $\mathrm{L}^{2}$ \\
{$\left[\psi_{2}^{0}\right]$} & $\mathrm{L}$ \\
{$\left[\psi_{3}^{0}\right]$} & 1 \\
{$\left[\psi_{4}^{0}\right]$} & $\mathrm{L}^{-1}$ \\
\hline &
\end{tabular}




\section{Foundations}

In this section, we review several of the key ideas and tools that are indispensable in our later discussions. We keep our explanations as concise as possible, and refrain from extensive proofs of any propositions. The reader will be directed to the appropriate references for the details. In large part, much of what is covered in this section should be familiar to many workers in GR.

\subsection{Asymptotic flatness and $\mathfrak{I}^{+}$}

Ever since the work of Bondi [16] illustrated the importance of null hypersurfaces in the study of outgoing gravitational radiation, the study of asymptotically-flat spacetimes has been one of the more important research topics in GR. Qualitatively speaking, a spacetime can be thought of as (future) asymptotically flat if the curvature tensor vanishes at an appropriate rate as infinity is approached along the future-directed null geodesics of the null hypersurfaces. The type of physical situation we have in mind is an arbitrary compact gravitating source (perhaps with an electric charge and current distribution), with the associated gravitational (and electromagnetic) field. The task is to gain information about the interior of the spacetime from the study of far-field features, multipole moments, gravitational and electromagnetic radiation, etc. [60]. The arena for this study is on what is referred to as future null infinity, $\mathfrak{I}^{+}$, the future boundary of the spacetime. The intuitive picture of this boundary is the set of all endpoints of future-directed null geodesics.

A precise definition of null asymptotic flatness and the boundary was given by Penrose [62, 63], whose basic idea was to rescale the spacetime metric by a conformal factor, which approaches zero asymptotically: the zero value defining future null infinity. This process leads to the boundary being a null hypersurface for the conformally-rescaled metric. When this boundary can be attached to the interior of the rescaled manifold in a regular way, then the spacetime is said to be asymptotically flat.

As the details of this formal structure are not used here, we will rely largely on the intuitive picture. A thorough review of this subject can be found in [23]. However, there are a number of important properties of $\mathfrak{I}^{+}$arising from Penrose's construction that we rely on [60, 62, 63]:

(A): For both the asymptotically-flat vacuum Einstein equations and the Einstein-Maxwell equations, $\mathfrak{I}^{+}$is a null hypersurface of the conformally rescaled metric.

(B): $\mathfrak{I}^{+}$is topologically $S^{2} \times \mathbb{R}$.

(C): The Weyl tensor $C_{b c d}^{a}$ vanishes at $\mathfrak{I}^{+}$, with the peeling theorem describing the speed of its falloff (see below).

Property (B) allows an easy visualization of the boundary, $\mathfrak{I}^{+}$, as the past light cone of the point $\boldsymbol{I}^{+}$, future timelike infinity. As mentioned earlier, $\mathfrak{I}^{+}$will be the stage for our study of asymptotically shear-free NGCs.

\subsection{Bondi coordinates and null tetrad}

Proceeding with our examination of the properties of $\mathfrak{I}^{+}$, we introduce, in the neighborhood of $\mathfrak{I}^{+}$, what is known as a Bondi coordinate system: $\left(u_{\mathrm{B}}, r, \zeta, \bar{\zeta}\right)$. In this system, $u_{\mathrm{B}}$, the Bondi time, labels the null surfaces, $r$ is the affine parameter along the null geodesics of the constant $u_{\mathrm{B}}$ surfaces and $\zeta=e^{i \phi} \cot (\theta / 2)$, the complex stereographic coordinate labeling the null geodesics of $\mathfrak{I}^{+}$. To reach $\mathfrak{I}^{+}$, we simply let $r \rightarrow \infty$, so that $\mathfrak{I}^{+}$has coordinates $\left(u_{\mathrm{B}}, \zeta, \bar{\zeta}\right)$. The time coordinate $u_{\mathrm{B}}$, the topologically $\mathbb{R}$ portion of $\mathfrak{I}^{+}$, labels 'cuts' of $\mathfrak{I}^{+}$. The stereographic coordinate $\zeta$ accounts for the topological generators of the $S^{2}$ portion of $\mathfrak{I}^{+}$, i.e., the null generators of $\mathfrak{I}^{+}$. The choice of a Bondi coordinate system is not unique, there being a variety of Bondi coordinate systems to choose from. The coordinate transformations between any two, known as Bondi-Metzner-Sachs (BMS) transformations or as the BMS group, are discussed later in this section. 
Associated with the Bondi coordinates is a (Bondi) null tetrad system, $\left(l^{a}, n^{a}, m^{a}, \bar{m}^{a}\right)$. The first tetrad vector $l^{a}$ is the tangent to the geodesics of the constant $u_{\mathrm{B}}$ null surfaces given by [60]

$$
\begin{aligned}
l^{a} & =\frac{d x^{a}}{d r}=g^{a b} \nabla_{b} u_{\mathrm{B}}, \\
l^{a} \nabla_{a} l^{b} & =0, \\
l^{a} \frac{\partial}{\partial x^{a}} & =\frac{\partial}{\partial r} .
\end{aligned}
$$

The second null vector $n^{a}$ is normalized so that:

$$
l_{a} n^{a}=1 .
$$

In Bondi coordinates, we have [60]

$$
n^{a} \frac{\partial}{\partial x^{a}}=\frac{\partial}{\partial u_{\mathrm{B}}}+U \frac{\partial}{\partial r}+X^{A} \frac{\partial}{\partial x^{A}},
$$

for functions $U$ and $X^{A}$ to be determined, and $A=\zeta, \bar{\zeta}$. At $\mathfrak{I}^{+}, n^{a}$ is tangent to the null generators of $\mathfrak{I}^{+}$.

The tetrad is completed with the choice of a complex null vector $m^{a},\left(m^{a} m_{a}=0\right)$ which is itself orthogonal to both $l_{a}$ and $n_{a}$, initially tangent to the constant $u_{\mathrm{B}}$ cuts at $\mathfrak{I}^{+}$and parallel propagated inward on the null geodesics. It is normalized by

$$
m^{a} \bar{m}_{a}=-1
$$

Once more, in coordinates, we have [60]

$$
m^{a} \frac{\partial}{\partial x^{a}}=\omega \frac{\partial}{\partial r}+\xi^{A} \frac{\partial}{\partial x^{A}}
$$

for some $\omega$ and $\xi^{A}$ to be determined. All other scalar products in the tetrad are to vanish.

With the tetrad thus defined, the contravariant metric of the spacetime is given by

$$
g^{a b}=l^{a} n^{b}+l^{b} n^{a}-m^{a} \bar{m}^{b}-m^{b} \bar{m}^{a} .
$$

In terms of the metric coefficients $U, \omega, X^{A}$, and $\xi^{A}$, the metric can be written as:

$$
\begin{aligned}
g^{a b} & =\left(\begin{array}{ccc}
0 & 1 & 0 \\
1 & g^{11} & g^{1 A} \\
0 & g^{1 A} & g^{A B}
\end{array}\right), \\
g^{11} & =2(U-\omega \bar{\omega}), \\
g^{1 A} & =X^{A}-\left(\bar{\omega} \xi^{A}+\omega \bar{\xi}^{A}\right), \\
g^{A B} & =-\left(\xi^{A} \bar{\xi}^{B}+\xi^{B} \bar{\xi}^{A}\right) .
\end{aligned}
$$

We thus have the spacetime metric in terms of the metric coefficients.

There remains the issue of both coordinate and tetrad freedom, i.e., local Lorentz transformations. Most of the time we work in one arbitrary but fixed Bondi coordinate system, though for special situations more general coordinate systems are used. The more general transformations are given, essentially, by choosing an arbitrary slicing of $\mathfrak{I}^{+}$, written as $u_{\mathrm{B}}=G(s, \zeta, \bar{\zeta})$ with $s$ labeling the slices. To keep conventional coordinate conditions unchanged requires a rescaling of 
$r: r \rightarrow r^{\prime}=\left(\partial_{s} G\right)^{-1} r$. It is also useful to be able to shift the origin of $r$ by $r^{\prime}=r-r_{0}\left(u_{\mathrm{B}}, \zeta, \bar{\zeta}\right)$ with arbitrary $r_{0}\left(u_{\mathrm{B}}, \zeta, \bar{\zeta}\right)$.

The tetrad freedom of null rotations around $n^{a}$, performed in the neighborhood of $\mathfrak{I}^{+}$, will later play a major role. For an arbitrary function $L\left(u_{\mathrm{B}}, \zeta, \bar{\zeta}\right)$ on $\mathfrak{I}^{+}$, the null rotation about the vector $n^{a}[60]$ is given by

$$
\begin{aligned}
l^{a} \rightarrow l^{* a} & =l^{a}-\frac{\bar{L}}{r} m^{a}-\frac{L}{r} \bar{m}^{a}+0\left(r^{-2}\right), \\
m^{a} \rightarrow m^{* a} & =m^{a}-\frac{L}{r} n^{a}+0\left(r^{-2}\right), \\
n^{a} \rightarrow n^{* a} & =n^{a} .
\end{aligned}
$$

Eventually, by the appropriate choice of the function $L\left(u_{\mathrm{B}}, \zeta, \bar{\zeta}\right)$, the new null vector, $l^{* a}$, can be made into the tangent vector of an asymptotically shear-free NGC.

A second type of tetrad transformation is the rotation in the tangent $\left(m^{a}, \bar{m}^{a}\right)$ plane, which keeps $l^{a}$ and $n^{a}$ fixed:

$$
m^{a} \rightarrow e^{i \lambda} m^{a}, \quad \lambda \in \mathbb{R} .
$$

This latter transformation provides motivation for the concept of spin weight. A quantity $\eta_{(s)}(\zeta, \bar{\zeta})$ is said to have spin-weight $s$ if, under the transformation, Eq. (2.13), it transforms as

$$
\eta \rightarrow \eta_{(s)}^{*}(\zeta, \bar{\zeta})=e^{i s \lambda} \eta_{(s)}(\zeta, \bar{\zeta}) .
$$

An example would be to take a vector on $\mathfrak{I}^{+}$, say $\eta^{a}$, and form the spin-weight-one quantity, $\eta_{(1)}=\eta^{a} m_{a}$.

Comment: For later use we note that $L\left(u_{\mathrm{B}}, \zeta, \bar{\zeta}\right)$ has spin weight, $s=1$.

For each $s$, spin- $s$ functions can be expanded in a complete basis set, the spin-s harmonics, ${ }_{s} Y_{l m}(\zeta, \bar{\zeta})$ or spin-s tensor harmonics, $Y_{l i \ldots j}^{(s)}(\zeta, \bar{\zeta}) \Leftrightarrow{ }_{s} Y_{l m}(\zeta, \bar{\zeta})$ (cf. Appendix C).

A third tetrad transformation, the boosts, are given by

$$
l^{\# a}=K l^{a}, \quad n^{\# a}=K^{-1} n^{a} .
$$

These transformations induce the idea of conformal weight, an idea similar to spin weight. Under a boost transformation, a quantity, $\eta_{(w)}$, will have conformal weight $w$ if

$$
\eta_{(w)} \rightarrow \eta_{(w)}^{\#}=K^{w} \eta_{(w)} .
$$

Sphere derivatives of spin-weighted functions $\eta_{(s)}(\zeta, \bar{\zeta})$ are given by the action of the operators $\partial$ and its conjugate operator $\overline{\bar{\partial}}$, defined by [28]

$$
\begin{gathered}
ð \eta_{(s)}=P^{1-s} \frac{\partial\left(P^{s} \eta_{(s)}\right)}{\partial \zeta}, \\
\bar{\partial} \eta_{(s)}=P^{1+s} \frac{\partial\left(P^{-s} \eta_{(s)}\right)}{\partial \bar{\zeta}},
\end{gathered}
$$

where the function $P$ is the conformal factor defining the conformal sphere metric,

$$
d s^{2}=\frac{4 d \zeta d \bar{\zeta}}{P^{2}},
$$

most often taken as the unit metric sphere by

$$
P=P_{0} \equiv 1+\zeta \bar{\zeta}
$$




\subsection{The optical equations}

Since this work concerns NGCs and, in particular, shear-free and asymptotically shear-free NGCs, it is necessary to first define them and then study their properties.

Given a Lorentzian manifold with local coordinates, $x^{a}$, and a NGC, i.e., a foliation by a three parameter family of null geodesics,

$$
x^{a}=X^{a}\left(r, y^{w}\right),
$$

with $r$ the affine parametrization and the (three) $y^{w}$ labeling the geodesics, the tangent vector field $l^{a}=D X^{a} \equiv \partial_{r} X^{a}$ satisfies the geodesic equation

$$
l^{a} \nabla_{a} l^{b}=0 .
$$

The two complex optical scalars (spin coefficients), $\rho$ and $\sigma$, are defined by

$$
\begin{aligned}
& \rho=\nabla_{a} l_{b} \bar{m}^{a} m^{b}, \\
& \sigma=\nabla_{(a} l_{b)} m^{a} m^{b}
\end{aligned}
$$

with $m^{a}$ an complex (spacelike) vector that is parallel propagated along the null geodesic field and satisfies $m^{a} m_{a}=m^{a} l_{a}=m^{a} \bar{m}_{a}+1=0$.

The $\rho$ and $\sigma$ satisfy the optical equations of Sachs [72], namely,

$$
\begin{aligned}
\frac{\partial \rho}{\partial r} & =\rho^{2}+\sigma \bar{\sigma}+\Phi_{00}, \\
\frac{\partial \sigma}{\partial r} & =(\rho+\bar{\rho}) \sigma+\psi_{0}, \\
\Phi_{00} & =R_{a b} l^{a} l^{b}, \\
\psi_{0} & =-C_{a b c d} l^{a} m^{b} l^{c} m^{d},
\end{aligned}
$$

where $\Phi_{00}$ and $\Psi_{0}$ are, respectively, a Ricci and a Weyl tensor tetrad component (see below). In flat space, i.e., with $\Phi_{00}=\Psi_{0}=0$, excluding the degenerate case of $\rho \bar{\rho}-\sigma \bar{\sigma}=0$, plane $(\rho=\sigma=0)$ and cylindrical fronts, the general solution is

$$
\begin{aligned}
& \rho=\frac{i \Sigma-r}{r^{2}+\Sigma^{2}-\sigma^{0} \bar{\sigma}^{0}}, \\
& \sigma=\frac{\sigma^{0}}{r^{2}+\Sigma^{2}-\sigma^{0} \bar{\sigma}^{0}} .
\end{aligned}
$$

The complex $\sigma^{0}$ (referred to as the asymptotic shear) and the real $\Sigma$ (called the twist) are determined from the original congruence, Eq. (2.19). Both are functions just of the parameters, $y^{w}$. Their behavior for large $r$ is given by

$$
\begin{aligned}
& \rho=-\frac{1}{r}+\frac{i \Sigma}{r^{2}}+\frac{\Sigma^{2}}{r^{3}}-\frac{\sigma^{0} \bar{\sigma}^{0}}{r^{3}}+O\left(r^{-4}\right), \\
& \sigma=\frac{\sigma^{0}}{r^{2}}+O\left(r^{-4}\right) .
\end{aligned}
$$

From this, $\sigma^{0}$ gets its name as the asymptotic shear. In Section 3, we return to the issue of the explicit construction of NGCs in Minkowski space and in particular to the construction and detailed properties of regular shear-free congruences. 
Note the important point that, in $\mathbb{M}$, the vanishing of the asymptotic shear forces the shear to vanish. The same is not true for asymptotically-flat spacetimes. Specifically, for future null asymptotically-flat spaces described in a Bondi tetrad and coordinate system, we have, from other considerations, that

$$
\begin{aligned}
\Phi_{00} & =O\left(r^{-6}\right), \\
\psi_{0} & =O\left(r^{-5}\right), \\
\Sigma & =0,
\end{aligned}
$$

which leads to the asymptotic behavior of $\rho$ and $\sigma$,

$$
\begin{aligned}
& \rho=\bar{\rho}=-\frac{1}{r}+\frac{\sigma^{0} \bar{\sigma}^{0}}{r^{3}}+O\left(r^{-5}\right), \\
& \sigma=\frac{\sigma^{0}}{r^{2}}+O\left(r^{-4}\right),
\end{aligned}
$$

with the two order symbols explicitly depending on the leading terms in $\Phi_{00}$ and $\Psi_{0}$. The vanishing of $\sigma^{0}$ does not, in this nonflat case, imply that $\sigma$ vanishes. This case, referred to as asymptotically shear-free, plays the major role later. It will be returned to in greater detail in Section 4.

\subsection{The Newman-Penrose formalism}

Though the NP formalism is the basic working tool for our analysis, this is not the appropriate venue for its detailed exposition. Instead we will simply give an outline of the basic ideas followed by the results found, from the application of the NP equations, to the problem of asymptotically-flat spacetimes.

The NP version $[55,60,57]$ of the vacuum Einstein (or the Einstein-Maxwell) equations uses the tetrad components

$$
\lambda_{i}^{a}=\left(l^{a}, n^{a}, m^{a}, \bar{m}^{a}\right),
$$

( $i=1,2,3,4)$ rather than the metric, as the basic variable. (An alternate version, not discussed here, is to use a pair of two-component spinors [66]) The metric, Eq. (2.8), can be written compactly as

$$
g^{a b}=\eta^{i j} \lambda_{i}^{a} \lambda_{j}^{b}
$$

with

$$
\eta^{i j}=\left(\begin{array}{cccc}
0 & 1 & 0 & 0 \\
1 & 0 & 0 & 0 \\
0 & 0 & 0 & -1 \\
0 & 0 & -1 & 0
\end{array}\right) .
$$

The complex spin coefficients, which play the role of the connection, are determined from the Ricci rotation coefficients $[55,60]$ :

$$
\gamma_{j k}^{i}=\lambda_{j}^{b} \lambda_{k}^{a} \nabla_{a} \lambda_{b}^{i} \equiv \lambda_{j}^{b} \lambda_{b ; k}^{i},
$$

via the linear combinations

$$
\begin{aligned}
& \alpha=\frac{1}{2}\left(\gamma_{124}-\gamma_{344}\right), \lambda=-\gamma_{244}, \kappa=\gamma_{131}, \\
& \beta=\frac{1}{2}\left(\gamma_{123}-\gamma_{343}\right), \mu=-\gamma_{243}, \rho=\gamma_{134}, \\
& \gamma=\frac{1}{2}\left(\gamma_{122}-\gamma_{342}\right), \nu=-\gamma_{242}, \sigma=\gamma_{133}, \\
& \varepsilon=\frac{1}{2}\left(\gamma_{121}-\gamma_{341}\right), \pi=-\gamma_{241}, \tau=\gamma_{132} .
\end{aligned}
$$


The third basic variable in the NP formalism is the Weyl tensor or, equivalently, the following five complex tetrad components of the Weyl tensor:

$$
\begin{gathered}
\psi_{0}=-C_{a b c d} l^{a} m^{b} l^{c} m^{d}, \quad \psi_{1}=-C_{a b c d} l^{a} n^{b} l^{c} m^{d}, \\
\psi_{2}=-\frac{1}{2}\left(C_{a b c d} l^{a} n^{b} l^{c} n^{d}-C_{a b c d} l^{a} n^{b} m^{c} \bar{m}^{d}\right), \\
\psi_{3}=C_{a b c d} l^{a} n^{b} n^{c} \bar{m}^{d}, \quad \psi_{4}=C_{a b c d} n^{a} \bar{m}^{b} n^{c} \bar{m}^{d} .
\end{gathered}
$$

Note that we have adopted the sign conventions of [55], which differ from those in [66].

When an electromagnetic field is present, we must include the complex tetrad components of the Maxwell field into the equations:

$$
\begin{aligned}
\phi_{0} & =F_{a b} l^{a} m^{b}, \\
\phi_{1} & =\frac{1}{2} F_{a b}\left(l^{a} n^{b}+m^{a} \bar{m}^{b}\right), \\
\phi_{2} & =F_{a b} n^{a} \bar{m}^{b},
\end{aligned}
$$

as well as the Ricci (or stress tensor) constructed from the three $\phi_{i}$, e.g., $T_{a b} l^{a} l^{b}=\phi_{0} \bar{\phi}_{0}$, with $R_{a b}=k T_{a b}, k=2 G c^{-4}$.

Remark 2. We mention that much of the physical content and interpretations in the present work comes from the study of the lowest spherical harmonic coefficients in the leading terms of the far-field expansions of the Weyl and Maxwell tensors.

The NP version of the vacuum (or Einstein-Maxwell) equations consists of three sets (or four sets) of nonlinear first-order coupled partial differential equations for the variables: the tetrad components, the spin coefficients, the Weyl tensor (and Maxwell field when present). Though there is no hope that they can be solved in any general sense, many exact solutions have been found from them. Of far more importance, large classes of asymptotic solutions and perturbation solutions can be found. Our interest lies in the asymptotic behavior of the asymptotically-flat solutions. Though there are some subtle issues, integration in this class is not difficult [55, 61]. With no explanation of the integration process, except to mention that we use the Bondi coordinate and tetrad system of Eqs. (2.3), (2.5), and (2.7) and asymptotic flatness $\left(\psi_{0} \sim O\left(r^{-5}\right)\right.$ and certain uniform smoothness conditions on sideways derivatives), we simply give the final results.

First, the radial behavior is described. The quantities with a zero superscript, e.g., $\sigma^{0}, \psi_{2}^{0}, \ldots$, are 'functions of integration', i.e., functions only of $\left(u_{\mathrm{B}}, \zeta, \bar{\zeta}\right)$.

- The Weyl tensor:

$$
\begin{aligned}
& \psi_{0}=\psi_{0}^{0} r^{-5}+O\left(r^{-6}\right), \\
& \psi_{1}=\psi_{1}^{0} r^{-4}+O\left(r^{-5}\right), \\
& \psi_{2}=\psi_{2}^{0} r^{-3}+O\left(r^{-4}\right), \\
& \psi_{3}=\psi_{3}^{0} r^{-2}+O\left(r^{-3}\right), \\
& \psi_{4}=\psi_{4}^{0} r^{-1}+O\left(r^{-2}\right) .
\end{aligned}
$$

- The Maxwell tensor:

$$
\begin{aligned}
& \phi_{0}=\phi_{0}^{0} r^{-3}+O\left(r^{-4}\right), \\
& \phi_{1}=\phi_{1}^{0} r^{-2}+O\left(r^{-3}\right), \\
& \phi_{2}=\phi_{2}^{0} r^{-1}+O\left(r^{-2}\right) .
\end{aligned}
$$


- The spin coefficients and metric variables:

$$
\begin{aligned}
\kappa & =\pi=\epsilon=0, \quad \tau=\bar{\alpha}+\beta, \\
\rho & =\bar{\rho}=-r^{-1}-\sigma^{0} \bar{\sigma}^{0} r^{-3}+O\left(r^{-5}\right), \\
\sigma & =\sigma^{0} r^{-2}+\left(\left(\sigma^{0}\right)^{2} \bar{\sigma}^{0}-\psi_{0}^{0} / 2\right) r^{-4}+O\left(r^{-5}\right), \\
\alpha & =\alpha^{0} r^{-1}+O\left(r^{-2}\right), \quad \beta=\beta^{0} r^{-1}+O\left(r^{-2}\right), \\
\gamma & =\gamma^{0}-\psi_{2}^{0}\left(2 r^{2}\right)^{-1}+O\left(r^{-3}\right), \quad \lambda=\lambda^{0} r^{-1}+O\left(r^{-2}\right), \\
\mu & =\mu^{0} r^{-1}+O\left(r^{-2}\right), \quad \nu=\nu^{0}+O\left(r^{-1}\right),
\end{aligned}
$$

$$
\begin{aligned}
A & =\zeta \text { or } \bar{\zeta} \\
\xi^{A} & =\xi^{0 A} r^{-1}-\sigma^{0} \bar{\xi}^{0 A} r^{-2}+\sigma^{0} \bar{\sigma}^{0} \xi^{0 A} r^{-3}+O\left(r^{-4}\right), \\
\omega & =\omega^{0} r^{-1}-\left(\sigma^{0} \bar{\omega}^{0}+\psi_{1}^{0} / 2\right) r^{-2}+O\left(r^{-3}\right), \\
X^{A} & =\left(\psi_{1}^{0} \bar{\xi}^{0 A}+\bar{\psi}_{1}^{0} \xi^{0 A}\right)\left(6 r^{3}\right)^{-1}+O\left(r^{-4}\right), \\
U & =U^{0}-\left(\gamma^{0}+\bar{\gamma}^{0}\right) r-\left(\psi_{2}^{0}+\bar{\psi}_{2}^{0}\right)(2 r)^{-1}+O\left(r^{-2}\right) .
\end{aligned}
$$

- The functions of integration are determined, using coordinate conditions, as:

$$
\begin{aligned}
\xi^{0 \zeta} & =-P, \quad \bar{\xi}^{0 \zeta}=0, \\
\xi^{0 \bar{\zeta}} & =0, \quad \bar{\xi}^{0 \bar{\zeta}}=-P, \\
P & =1+\zeta \bar{\zeta}, \\
\alpha^{0} & =-\bar{\beta}^{0}=-\frac{\zeta}{2}, \\
\gamma^{0} & =\nu^{0}=0, \\
\omega^{0} & =-\bar{\partial} \sigma^{0}, \\
\lambda^{0} & =\dot{\bar{\sigma}}^{0} \\
\mu^{0} & =U^{0}=-1, \\
\psi_{4}^{0} & =-\ddot{\bar{\sigma}}^{0}, \\
\psi_{3}^{0} & =\check{\partial}^{0}, \\
\overline{\bar{\sigma}}^{0} & =\overline{\bar{\partial}}^{2} \sigma^{0}-\check{\partial}^{2} \bar{\sigma}^{0}+\bar{\sigma}^{0} \lambda^{0}-\sigma^{0} \bar{\lambda}^{0} .
\end{aligned}
$$

- The mass aspect,

$$
\Psi \equiv \psi_{2}^{0}+\check{\partial}^{2} \bar{\sigma}^{0}+\sigma^{0} \dot{\bar{\sigma}}^{0}
$$

satisfies the physically very important reality condition:

$$
\Psi=\bar{\Psi}
$$

- Finally, from the asymptotic Bianchi identities, we obtain the dynamical (or evolution) relations: 


$$
\begin{aligned}
\dot{\psi}_{2}^{0} & =-ð \psi_{3}^{0}+\sigma^{0} \psi_{4}^{0}+k \phi_{2}^{0} \bar{\phi}_{2}^{0}, \\
\dot{\psi}_{1}^{0} & =-ð \psi_{2}^{0}+2 \sigma^{0} \psi_{3}^{0}+2 k \phi_{1}^{0} \bar{\phi}_{2}^{0}, \\
\dot{\psi}_{0}^{0} & =-ð \psi_{1}^{0}+3 \sigma^{0} \psi_{2}^{0}+3 k \phi_{0}^{0} \bar{\phi}_{2}^{0}, \\
\dot{\phi}_{1}^{0} & =-ð \phi_{2}^{0}, \\
\dot{\phi}_{0}^{0} & =-ð \phi_{1}^{0}+\sigma^{0} \phi_{2}^{0} ; \\
k & =2 G c^{-4} .
\end{aligned}
$$

Remark 3. These last five equations, the first of which contains the beautiful Bondi energymomentum loss theorem, play the fundamental role in the dynamics of our physical quantities.

Remark 4. Using the mass aspect, $\Psi$, with Eqs. (2.47) and (2.48), the first of the asymptotic Bianchi identities, Eq. (2.52), can be rewritten in the concise form,

$$
\dot{\Psi}=\dot{\sigma} \dot{\bar{\sigma}}+k \phi_{2}^{0} \bar{\phi}_{2}^{0} .
$$

From these results, the characteristic initial problem can roughly be stated in the following manner. At $u_{\mathrm{B}}=u_{\mathrm{B} 0}$ we choose the initial values for $\left(\psi_{0}^{0}, \psi_{1}^{0}, \psi_{2}^{0}\right)$, i.e., functions only of $(\zeta, \bar{\zeta})$. The characteristic data, the complex Bondi shear, $\sigma^{0}\left(u_{\mathrm{B}}, \zeta, \bar{\zeta}\right)$, is then freely chosen. Since $\psi_{3}^{0}$ and $\psi_{4}^{0}$ are functions of $\sigma^{0}$, Eqs. (2.45), (2.47) and (2.48) and its derivatives, all the asymptotic variables can now be determined from Eqs. (2.52)-(2.56).

An important consequence of the NP formalism is that it allows simple proofs for many geometric theorems. Two important examples are the Goldberg-Sachs theorem [29] and the peeling theorem [73]. The peeling theorem is essentially given by the asymptotic behavior of the Weyl tensor in Eq. (2.36) (and Eq. (2.37)). The Goldberg-Sachs theorem is discussed in some detail in Section 2.6. Both theorems are implicitly used later.

One of the immediate physical interpretations arising from the asymptotically-flat solutions was Bondi's [16] identifications, at $\mathfrak{I}^{+}$, of the interior spacetime four-momentum (energy/momentum). Given the mass aspect, Eq. (2.50),

$$
\Psi=\psi_{2}^{0}+ð^{2} \bar{\sigma}^{0}+\sigma^{0} \dot{\bar{\sigma}}^{0},
$$

and the spherical harmonic expansion

$$
\Psi=\Psi^{0}+\Psi^{i} Y_{1 i}^{0}+\Psi^{i j} Y_{2 i j}^{0}+\ldots,
$$

Bondi identified the interior mass and three-momentum with the $l=0$ and $l=1$ harmonic contributions;

$$
\begin{gathered}
M_{\mathrm{B}}=-\frac{c^{2}}{2 \sqrt{2} G} \Psi^{0}, \\
P^{i}=-\frac{c^{3}}{6 G} \Psi^{i} .
\end{gathered}
$$

The evolution of these quantities, (the Bondi mass/momentum loss) is then determined from Eq. (2.58). The details of this will be discussed in Section 5.

The same clear cut asymptotic physical identification for interior angular momentum is not as readily available. In vacuum linear theory, the angular momentum is often taken to be

$$
J^{k}=-\frac{\sqrt{2} c^{3}}{12 G} \operatorname{Im}\left(\psi_{1}^{0 k}\right) .
$$


However, in the nonlinear treatment, correction terms quadratic in $\sigma^{0}$ and its derivatives are often included [75]. In the presence of a Maxwell field, this is again modified by the addition of electromagnetic multipole terms [42, 4].

In our case, where we consider only quadrupole gravitational radiation, the quadratic correction terms do in fact vanish and hence Eq. (2.62), modified by the Maxwell terms, is correct as it is stated.

\subsection{The Bondi-Metzner-Sachs group}

The group of coordinate transformations at $\mathfrak{I}^{+}$that preserves the Bondi coordinate conditions, the BMS group, is the same as the asymptotic symmetry group that arises from approximate solutions to Killing's equation as $\mathfrak{I}^{+}$is approached. The BMS group has two parts: the homogeneous Lorentz group and the supertranslation group, which contains the Poincaré translation sub-group. Their importance to us lies in the fact that all the physical quantities arising from our identifications must transform appropriately under these transformations [65, 42].

Specifically, the BMS group is given by the supertranslations, with $\alpha(\zeta, \bar{\zeta})$ an arbitrary regular differentiable function on $S^{2}$ :

$$
\begin{aligned}
\widehat{u}_{B} & =u_{\mathrm{B}}+\alpha(\zeta, \bar{\zeta}) \\
(\widehat{\zeta}, \widehat{\zeta}) & =(\zeta, \bar{\zeta})
\end{aligned}
$$

and the Lorentz transformations, with $(a, b, c, d)$ the complex parameters of $\mathrm{SL}(2, \mathbb{C})$,

$$
\begin{aligned}
\widehat{u}_{B} & =K u_{\mathrm{B}}, \\
K & =\frac{1+\zeta \bar{\zeta}}{(a \zeta+b)(\bar{a} \bar{\zeta}+\bar{b})+(c \zeta+d)(\bar{c} \bar{\zeta}+\bar{d})}, \\
\widehat{\zeta} & =\frac{a \zeta+b}{c \zeta+d}, \quad a d-b c=1 .
\end{aligned}
$$

If $\alpha(\zeta, \bar{\zeta})$ is expanded in spherical harmonics,

$$
\alpha(\zeta, \bar{\zeta})=\sum_{m, l} \alpha^{m l} Y_{l m}(\zeta, \bar{\zeta}),
$$

the $l=0,1$ terms represent the Poincaré translations, i.e.,

$$
\alpha_{(P)}(\zeta, \bar{\zeta})=d^{a} \hat{l}_{a}=\frac{\sqrt{2}}{2} d^{0} Y_{0}^{0}-\frac{1}{2} d^{i} Y_{1 i}^{0} .
$$

Details about the representation theory, with applications, are given later.

\subsection{Algebraically-special metrics and the Goldberg-Sachs theorem}

Among the most studied vacuum spacetimes are those referred to as 'algebraically-special' spacetimes, i.e., vacuum spacetimes that possess two or more coinciding principal null direction (PND) vectors. PND fields [66] (in general, four locally-independent fields exist) are defined by solutions, $L^{a}$, to the algebraic equation

$$
L^{b} L_{[e} C_{a] b c[d} L_{f]} L^{c}=0, \quad L^{a} L_{a}=0 .
$$


The Cartan-Petrov-Pirani-Penrose classification $[68,69,66]$ describes the different degeneracies (i.e., the number of coinciding PNDs):

$$
\begin{array}{ll}
\text { Alg. General } & {[1,1,1,1]} \\
\text { Type II } & {[2,1,1]}
\end{array}
$$

Type $\mathrm{D}$ or degenerate $[2,2]$

Type III $\quad[3,1]$

Type IV or N [4].

In NP language, if the tetrad vector $l^{a}$ is a principal null direction, i.e., $L_{a}=l_{a}$, then automatically,

$$
\psi_{0}=0
$$

For the algebraically-special metrics, the special cases are

$$
\begin{aligned}
& \text { Type II } \psi_{0}=\psi_{1}=0 \\
& \text { Type III } \psi_{0}=\psi_{1}=\psi_{2}=0 \\
& \text { Type IV } \psi_{0}=\psi_{1}=\psi_{2}=\psi_{3}=0 \\
& \text { Type D } \begin{array}{l}
\psi_{0}=\psi_{1}=\psi_{3}=\psi_{4}=0 \\
\text { with both } l^{a} \text { and } n^{a} \text { PNDs. }
\end{array}
\end{aligned}
$$

An outstanding feature of the algebraically-special metrics is contained in the beautiful GoldbergSachs theorem [29].

Theorem (Goldberg-Sachs). For a nonflat vacuum spacetime, if there is an NGC that is shearfree, i.e., there is a null vector field with $(\kappa=0, \sigma=0)$, then the spacetime is algebraically special and, conversely, if a vacuum spacetime is algebraically special, there is an NGC with $(\kappa=0, \sigma=0)$.

In particular, this means that for all algebraically special metrics there is an everywhere shearfree NGC, and a null tetrad exists such that $\psi_{0}=\psi_{1}=0$. The main idea of this review is an asymptotic generalization of this statement: for all asymptotically flat metrics, there exists a null tetrad such that the $l=0$ and $l=1$ harmonic coefficients of the asymptotic Weyl tensor components $\psi_{0}$ and $\psi_{1}$ (namely, $\psi_{0}^{0 i}$ and $\psi_{1}^{0 i}$ ) vanish. Note that this is in reality a nontrivial condition only on $\psi_{1}^{0 i}$, since the other three components vanish automatically when we recall that $\psi_{0}$ and $\psi_{1}$ are spin-weight two and one respectively. 


\section{Shear-Free NGCs in Minkowski Space}

The structure and properties of asymptotically shear-free NGCs (our main topic) are best understood by first looking at the special case of congruences that are shear-free everywhere (except at their caustics). Though shear-free congruences are also found in algebraically-special spacetimes, in this section only the shear-free NGCs in Minkowski spacetime, $\mathbb{M}$, are discussed [7]

\subsection{The flat-space good-cut equation and good-cut functions}

In Section 2, we saw that in the NP formalism, two of the complex spin coefficients, the optical parameters $\rho$ and $\sigma$ of Eqs. (2.23) and (2.24), play a particularly important role in their description of an NGC; namely, they carry the information of the divergence, twist and shear of the congruence.

From Eqs. (2.23) and (2.24), the radial behavior of the optical parameters for general shear-free NGCs, in Minkowski space, is given by

$$
\rho=\frac{i \Sigma-r}{r^{2}+\Sigma^{2}}, \quad \sigma=0
$$

where $\Sigma$ is the twist of the congruence. A more detailed and much deeper understanding of the shear-free congruences can be obtained by first looking at the explicit coordinate expression, Eq. (2.19), for all flat-space NGCs:

$$
x^{a}=u_{\mathrm{B}}\left(\hat{l}^{a}+\hat{n}^{a}\right)-L \overline{\hat{m}}^{a}-\bar{L} \hat{m}^{a}+\left(r^{*}-r_{0}\right) \hat{l}^{a},
$$

where $L\left(u_{\mathrm{B}}, \zeta, \bar{\zeta}\right)$ is an arbitrary complex function of the parameters $y^{w}=\left(u_{\mathrm{B}}, \zeta, \bar{\zeta}\right) ; r_{0}$, also an arbitrary function of $\left(u_{\mathrm{B}}, \zeta, \bar{\zeta}\right)$, determines the origin of the affine parameter; and $r^{*}$ can be chosen freely. Most frequently, to simplify the form of $\rho, r_{0}$ is chosen as

$$
r_{0} \equiv-\frac{1}{2}(ð \bar{L}+\bar{\partial} L+L \dot{\bar{L}}+\bar{L} \dot{L})
$$

At this point, Eq. (3.2) describes an arbitrary NGC with $\left(u_{\mathrm{B}}, \zeta, \bar{\zeta}\right)$ labeling the geodesics and $r^{*}$ the affine distance along the individual geodesics; later $L\left(u_{\mathrm{B}}, \zeta, \bar{\zeta}\right)$ will be chosen so that the congruence is shear-free. The tetrad $\left(\hat{l}^{a}, \hat{n}^{a}, \hat{m}^{a}, \overline{\hat{m}}^{a}\right)$ is given by Eqs. $(1.1-1.2)$, see [40].

There are several important comments to be made about Eq. (3.2). The first is that there is a simple geometric meaning to the parameters $\left(u_{\mathrm{B}}, \zeta, \bar{\zeta}\right)$ : they are the values of the Bondi coordinates of $\mathfrak{I}^{+}$, where each geodesic of the congruence intersects $\mathfrak{I}^{+}$. The second concerns the geometric meaning of $L$. At each point of $\mathfrak{I}^{+}$, consider the past light cone and its sphere of null directions. Coordinatize that sphere (of null directions) with stereographic coordinates. The function $L\left(u_{\mathrm{B}}, \zeta, \bar{\zeta}\right)$ is the stereographic angle field on $\mathfrak{I}^{+}$that describes the null direction of each geodesic intersecting $\mathfrak{I}^{+}$at the point $\left(u_{\mathrm{B}}, \zeta, \bar{\zeta}\right)$. The values $L=0$ and $L=\infty$ represent, respectively, the direction along the Bondi $l^{a}$ and $n^{a}$ vectors. This stereographic angle field completely determines the NGC.

The twist, $\Sigma$, of the congruence can be calculated in terms of $L\left(u_{\mathrm{B}}, \zeta, \bar{\zeta}\right)$ directly from Eq. (3.2) and the definition of the complex divergence, Eq. (2.20), leading to

$$
i \Sigma=\frac{1}{2}\{ð \bar{L}+L \dot{\bar{L}}-\overline{\mathrm{\partial}} L-\bar{L} \dot{L}\}
$$

We now demand that $L$ be a regular function of its arguments (i.e., have no infinities), or, equivalently, that all members of the NGC come from the interior of the spacetime and not lie on $\mathfrak{I}^{+}$ itself. 
It has been shown [12] that the condition on the stereographic angle field $L$ for the NGC to be shear-free is that

$$
ð L+L \dot{L}=0 .
$$

Our task is now to find the regular solutions of Eq. (3.5). The key to doing this is via the introduction of a new complex variable $\tau$ and complex function [39, 40],

$$
\tau=T\left(u_{\mathrm{B}}, \zeta, \bar{\zeta}\right)
$$

$T$ is related to $L$ by the $\mathrm{CR}$ equation (related to the existence of a $\mathrm{CR}$ structure on $\mathfrak{I}^{+}$; see Appendix B):

$$
\mathrm{\partial}_{\left(u_{\mathrm{B}}\right)} T+L \dot{T}=0 .
$$

Remark 5. The following 'gauge' freedom becomes useful later. $\tau \rightarrow \tau^{*}=F(\tau)$, with $F$ analytic, leaving Eq. (3.7) unchanged. In other words,

$$
\tau^{*}=T^{*}\left(u_{\mathrm{B}}, \zeta, \bar{\zeta}\right) \equiv F\left(T\left(u_{\mathrm{B}}, \zeta, \bar{\zeta}\right)\right)
$$

leads to

$$
\begin{aligned}
\varlimsup_{\left(u_{\mathrm{B}}\right)} T^{*} & =F^{\prime} \check{\partial}_{\left(u_{\mathrm{B}}\right)} T, \\
\dot{T}^{*} & =F^{\prime} \dot{T}, \\
\mathrm{\partial}_{\left(u_{\mathrm{B}}\right)} T^{*}+L \dot{T}^{*} & =0 .
\end{aligned}
$$

We assume, in the neighborhood of real $\mathfrak{I}^{+}$, i.e., near the real $u_{\mathrm{B}}$ and $\tilde{\zeta}=\bar{\zeta}$, that $T\left(u_{\mathrm{B}}, \zeta, \tilde{\zeta}\right)$ is analytic in the three arguments $\left(u_{\mathrm{B}}, \zeta, \tilde{\zeta}\right)$. The inversion of Eq. (3.6) yields the complex analytic cut function

$$
u_{\mathrm{B}}=G(\tau, \zeta, \tilde{\zeta}) .
$$

Though we are interested in real values for $u_{\mathrm{B}}$, from Eq. (3.9) we see that for arbitrary $\tau$ it may take complex values. Shortly, we will also address the important issue of what values of $\tau$ are needed for real $u_{\mathrm{B}}$.

Returning to the issue of integrating the shear-free condition, Eq. (3.5), using Eq. (3.6), we note that the derivatives of $T, \mathrm{\partial}_{\left(u_{\mathrm{B}}\right)} T$ and $\dot{T}$ can be expressed in terms of the derivatives of $G(\tau, \zeta, \bar{\zeta})$ by implicit differentiation. The $u_{\mathrm{B}}$ derivative of $T$ is obtained by taking the $u_{\mathrm{B}}$ derivative of Eq. (3.9):

$$
1=G^{\prime}(\tau, \zeta, \bar{\zeta}) \dot{T} \Rightarrow \dot{T}=\frac{1}{\left(G^{\prime}\right)},
$$

while the $\widetilde{\partial}_{\left(u_{\mathrm{B}}\right)} T$ derivative is found by applying $\check{\partial}_{\left(u_{\mathrm{B}}\right)}$ to Eq. (3.9),

$$
\begin{aligned}
0 & =G^{\prime}(\tau, \zeta, \bar{\zeta}) \partial_{\left(u_{\mathrm{B}}\right)} T+\mathrm{\partial}_{(\tau)} G, \\
\varlimsup_{\left(u_{\mathrm{B}}\right)} T & =-\frac{\mathrm{\partial}_{(\tau)} G}{G^{\prime}(\tau, \zeta, \bar{\zeta})} .
\end{aligned}
$$

When Eqs. (3.10) and (3.11) are substituted into Eq. (3.7), one finds that $L$ is given implicitly in terms of the cut function by

$$
\begin{aligned}
L\left(u_{\mathrm{B}}, \zeta, \bar{\zeta}\right) & =ð_{(\tau)} G(\tau, \zeta, \bar{\zeta}), \\
u_{\mathrm{B}} & =G(\tau, \zeta, \bar{\zeta}) \Leftrightarrow \tau=T\left(u_{\mathrm{B}}, \zeta, \bar{\zeta}\right) .
\end{aligned}
$$

Thus, we see that all information about the NGC can be obtained from the cut function $G(\tau, \zeta, \bar{\zeta})$. 
By further implicit differentiation of Eq. (3.12), i.e.,

$$
\begin{aligned}
\partial_{\left(u_{\mathrm{B}}\right)} L\left(u_{\mathrm{B}}, \zeta, \bar{\zeta}\right) & =\partial_{(\tau)}^{2} G(\tau, \zeta, \bar{\zeta})+\partial_{(\tau)} G^{\prime}(\tau, \zeta, \bar{\zeta}) \cdot \partial_{\left(u_{\mathrm{B}}\right)} T, \\
\dot{L}\left(u_{\mathrm{B}}, \zeta, \bar{\zeta}\right) & =\partial_{(\tau)} G^{\prime}(\tau, \zeta, \bar{\zeta}) \cdot \dot{T},
\end{aligned}
$$

using Eq. (3.7), the shear-free condition (3.5) becomes

$$
\mathrm{\partial}_{(\tau)}^{2} G(\tau, \zeta, \bar{\zeta})=0 .
$$

This equation will be referred to as the homogeneous Good-Cut Equation and its solutions as flatspace Good-Cut Functions (GCFs). In the next Section 4, an inhomogeneous version, the Good-Cut Equation, will be found for asymptotically shear-free NGCs. Its solutions will also be referred to as GCFs.

From the properties of the $\partial^{2}$ operator, the general regular solution to Eq. (3.14) is easily found: $G$ must contain only $l=0$ and $l=1$ spherical harmonic contributions; thus, any regular solution will be dependent on four arbitrary complex parameters, $z^{a}$. If these parameters are functions of $\tau$, i.e., $z^{a}=\xi^{a}(\tau)$, then we can express any regular solution $G$ in terms of the complex world line $\xi^{a}(\tau)[39,40]:$

$$
u_{\mathrm{B}}=G(\tau, \zeta, \bar{\zeta})=\xi^{a}(\tau) \hat{l}_{a}(\zeta, \bar{\zeta}) \equiv \frac{\sqrt{2} \xi^{0}(\tau)}{2}-\frac{1}{2} \xi^{i}(\tau) Y_{1 i}^{0} .
$$

The angle field $L\left(u_{\mathrm{B}}, \zeta, \bar{\zeta}\right)$ then has the form

$$
\begin{aligned}
L\left(u_{\mathrm{B}}, \zeta, \bar{\zeta}\right) & =\partial_{(\tau)} G(\tau, \zeta, \bar{\zeta})=\xi^{a}(\tau) \hat{m}_{a}(\zeta, \bar{\zeta}), \\
u_{\mathrm{B}} & =\xi^{a}(\tau) \hat{l}_{a}(\zeta, \bar{\zeta}) .
\end{aligned}
$$

Thus, we have our first major result: every regular shear-free NGC in Minkowski space is generated by the arbitrary choice of a complex world line in what turns out to be complex Minkowski space. See Eq. (2.66) for the connection between the $l=(0,1)$ harmonics in Eq. (3.15) and the Poincaré translations. We see in the next Section 4 how this result generalizes to regular asymptotically shear-free NGCs.

Remark 6. We point out that this construction of regular shear-free NGCs in Minkowski space is a special example of the Kerr theorem (cf. [67]). Writing Eqs. (3.16) and (3.17) as

$$
\begin{aligned}
u_{\mathrm{B}} & =\frac{a+b \bar{\zeta}+\bar{b} \zeta+c \zeta \bar{\zeta}}{1+\zeta \bar{\zeta}}, \\
L & =\frac{(\bar{b}+c \bar{\zeta})-\bar{\zeta}(a+b \bar{\zeta})}{1+\bar{\zeta}},
\end{aligned}
$$

where the $(a(\tau), b(\tau), c(\tau), d(\tau))$ are simple combinations of the $\xi^{a}(\tau)$, we then find that

$$
\begin{aligned}
L+u_{B} \bar{\zeta} & =\bar{b}+c \bar{\zeta}, \\
u_{B}-L \zeta & =a+b \bar{\zeta} .
\end{aligned}
$$

Noting that the right-hand side of both equations are functions only of $\tau$ and $\bar{\zeta}$, we can eliminate the $\tau$ from the two equations, thereby constructing a function of three variables of the form

$$
F\left(L+u_{B} \bar{\zeta}, u_{B}-L \zeta, \bar{\zeta}\right)=0 .
$$

This is a special case of the general solution to Eq. (3.5), which is the Kerr theorem.

In addition to the construction of the angle field, $L\left(u_{\mathrm{B}}, \zeta, \bar{\zeta}\right)$, from the GCF, another quantity of great value in applications, obtained from the GCF, is the local change in $u_{\mathrm{B}}$ as $\tau$ changes, i.e.,

$$
V(\tau, \zeta, \tilde{\zeta}) \equiv \partial_{\tau} G=G^{\prime} .
$$




\subsection{Real cuts from the complex good cuts, I}

Though our discussion of shear-free NGCs has relied, in an essential manner, on the use of the complexification of $\mathfrak{I}^{+}$and the complex world lines in complex Minkowski space, it is the real structures that are of main interest to us. We want to find the intersection of the complex GCF with real $\mathfrak{I}^{+}$, i.e., what are the real points and real cuts of $u_{\mathrm{B}}=G(\tau, \zeta, \bar{\zeta}),(\tilde{\zeta}=\bar{\zeta})$, and what are the values of $\tau$ that yield real $u_{\mathrm{B}}$. These reality structures were first observed in [7] and recently there have been attempts to study them in the framework of holographic dualities (cf. [8] and Section 8).

To construct an associated family of real cuts from a GCF, we begin with

$$
u_{\mathrm{B}}=G(\tau, \zeta, \bar{\zeta})=\frac{\sqrt{2}}{2} \xi^{0}(\tau)-\frac{1}{2} \xi^{i}(\tau) Y_{1 i}^{0}(\zeta, \bar{\zeta})
$$

and write

$$
\tau=s+i \lambda
$$

with $s$ and $\lambda$ real. The cut function can then be rewritten

$$
\begin{aligned}
u_{\mathrm{B}} & =G(\tau, \zeta, \bar{\zeta})=G(s+i \lambda, \zeta, \bar{\zeta}) \\
& =G_{R}(s, \lambda, \zeta, \bar{\zeta})+i G_{I}(s, \lambda, \zeta, \bar{\zeta}),
\end{aligned}
$$

with real $G_{R}(s, \lambda, \zeta, \bar{\zeta})$ and $G_{I}(s, \lambda, \zeta, \bar{\zeta})$. The $G_{R}(s, \lambda, \zeta, \bar{\zeta})$ and $G_{I}(s, \lambda, \zeta, \bar{\zeta})$ are easily calculated from $G(\tau, \zeta, \bar{\zeta})$ by

$$
\begin{aligned}
G_{R}(s, \lambda, \zeta, \bar{\zeta}) & =\frac{1}{2}\{G(s+i \lambda, \zeta, \bar{\zeta})+\overline{G(s+i \lambda, \zeta, \bar{\zeta})}\} \\
G_{I}(s, \lambda, \zeta, \bar{\zeta}) & =-\frac{i}{2}\{G(s+i \lambda, \zeta, \bar{\zeta})-\overline{G(s+i \lambda, \zeta, \bar{\zeta})}\}
\end{aligned}
$$

By setting

$$
G_{I}(s, \lambda, \zeta, \bar{\zeta})=0
$$

and solving for

$$
\lambda=\Lambda(s, \zeta, \bar{\zeta})
$$

we obtain the associated one-parameter, $s$, family of real slicings,

$$
u_{\mathrm{B}}^{(R)}=G_{R}(s, \Lambda(s, \zeta, \bar{\zeta}), \zeta, \bar{\zeta})=\xi^{a}(s+i \Lambda(s, \zeta, \bar{\zeta})) l_{a}(\zeta, \bar{\zeta}) .
$$

Thus, the values of $\tau$ that yield real values of $u_{\mathrm{B}}$ are given by

$$
\tau=s+i \Lambda(s, \zeta, \bar{\zeta}) .
$$

Perturbatively, using Eq. (3.19) and writing $\xi^{a}(s)=\xi_{R}^{a}(s)+i \xi_{I}^{a}(s)$, we find $\lambda$ to first order:

$$
\begin{aligned}
u_{\mathrm{B}}= & \frac{\sqrt{2}}{2} \xi_{R}^{0}(s)-\frac{\sqrt{2}}{2} \xi_{I}^{0}(s)^{\prime} \lambda-\frac{1}{2}\left[\xi_{R}^{i}(s)-\xi_{I}^{i}(s)^{\prime} \lambda\right] Y_{1 i}^{0}(\zeta, \bar{\zeta}) \\
& +i\left[\frac{\sqrt{2}}{2} \xi_{I}^{0}(s)+\frac{\sqrt{2}}{2} \xi_{R}^{0}(s)^{\prime} \lambda\right]-i \frac{1}{2}\left[\xi_{I}^{i}(s)+\xi_{R}^{i}(s)^{\prime} \lambda\right] Y_{1 i}^{0}(\zeta, \bar{\zeta}), \\
u_{\mathrm{B}}^{(R)}= & G_{R}(s, \Lambda, \zeta, \bar{\zeta}) \\
= & \frac{\sqrt{2}}{2} \xi_{R}^{0}(s)-\frac{\sqrt{2}}{2} \xi_{I}^{0}(s)^{\prime} \lambda-\frac{1}{2}\left[\xi_{R}^{i}(s)-\xi_{I}^{i}(s)^{\prime} \lambda\right] Y_{1 i}^{0}(\zeta, \bar{\zeta}), \\
\lambda= & \Lambda(s, \zeta, \bar{\zeta})=-\frac{\sqrt{2} \xi_{I}^{0}(s)+\xi_{I}^{i}(s) Y_{1 i}^{0}(\zeta, \bar{\zeta})}{\left[\sqrt{2} \xi_{R}^{0}(s)^{\prime}-\xi_{R}^{i}(s)^{\prime} Y_{1 i}^{0}(\zeta, \bar{\zeta})\right]}
\end{aligned}
$$


Continuing, with small values for the imaginary part of $\xi^{a}(\tau)=\xi_{R}^{a}(\tau)+i \xi_{I}^{a}(\tau),\left(\xi_{R}^{a}(\tau), \xi_{I}^{a}(\tau)\right.$ both real analytic functions) and hence small $\Lambda(s, \zeta, \bar{\zeta})$, it is easy to see that $\Lambda(s, \zeta, \bar{\zeta})$ (for fixed value of $s$ ) is a bounded smooth function on the $(\zeta, \bar{\zeta})$ sphere, with maximum and minimum values, $\lambda_{\max }=\Lambda\left(s, \zeta_{\max }, \bar{\zeta}_{\max }\right)$ and $\lambda_{\min }=\Lambda\left(s, \zeta_{\min }, \bar{\zeta}_{\min }\right)$. Furthermore on the $(\zeta, \bar{\zeta})$ sphere, there are a finite line-segments worth of curves (circles) that lie between $\left(\zeta_{\min }, \bar{\zeta}_{\min }\right)$ and $\left(\zeta_{\max }, \bar{\zeta}_{\max }\right)$ such that $\Lambda(s, \zeta, \bar{\zeta})$ is a monotonically increasing function on the family of curves. Hence there will be a family of circles on the $(\zeta, \bar{\zeta})$-sphere where the value of $\lambda$ is a constant, ranging between $\lambda_{\max }$ and $\lambda_{\min }$

Summarizing, we have the result that in the complex $\tau$-plane there is a ribbon or strip given by all values of $s$ and line segments parametrized by $\lambda$ between $\lambda_{\min }$ and $\lambda_{\max }$ such that the complex light-cones from each of the associated points, $\xi^{a}(s+i \lambda)$, all have some null geodesics that intersect real $\mathfrak{I}^{+}$. More specifically, for each of the allowed values of $\tau=s+i \lambda$ there will be a circle's worth of complex null geodesics leaving the point $\xi^{a}(s+i \lambda)$ reaching real $\mathfrak{I}^{+}$. It is the union of these null geodesics, corresponding to the circles on the $(\zeta, \bar{\zeta})$-sphere from the line segment, that produces the real family of cuts, Eq. (3.26).

The real structure associated with a complex world line is then this one-parameter family of slices (cuts) Eq. (3.26).

Remark 7. We saw earlier that the shear-free angle field was given by

$$
\begin{aligned}
L\left(u_{\mathrm{B}}, \zeta, \bar{\zeta}\right) & =\varlimsup_{(\tau)} G(\tau, \zeta, \bar{\zeta}), \\
u_{\mathrm{B}} & =G(\tau, \zeta, \bar{\zeta}) \Leftrightarrow \tau=T\left(u_{\mathrm{B}}, \zeta, \bar{\zeta}\right),
\end{aligned}
$$

where real values of $u_{\mathrm{B}}$ should be used. If the real cuts, $u_{\mathrm{B}}=G_{R}(s, \Lambda(s, \zeta, \bar{\zeta}), \zeta, \bar{\zeta})$, were used instead to calculate $L\left(u_{\mathrm{B}}, \zeta, \bar{\zeta}\right)$, the results would be wrong. The restriction of $\tau$ to yield real $u_{\mathrm{B}}$, does not commute with the application of the operator, i.e.,

$$
L\left(u_{\mathrm{B}}, \zeta, \bar{\zeta}\right) \neq \varnothing G_{R} .
$$

The $\partial$ differentiation must be done first, holding $\tau$ constant, before the reality of $u_{\mathrm{B}}$ is used. In other words, though we are interested in real $\mathfrak{I}^{+}$, it is essential that we consider its (local) complexification.

There are a pair of important (dual) results that arise from the considerations of the good cuts $[7,8]$. From the stereographic angle field, i.e., $L$ from Eqs. (3.31) and (3.32), one can form two different conjugate fields, (1) the complex conjugate of $L$ :

$$
\bar{L}=\bar{ळ}_{\bar{\tau}} \bar{G}=\bar{\xi}^{a}(\bar{\tau}) \overline{\hat{m}}_{a}
$$

and (2) the holomorphic conjugate, $\widetilde{L}$, given by

$$
\widetilde{L}=\bar{\delta}_{\tau} G=\xi^{a}(\tau) \overline{\hat{m}}_{a} .
$$

The two different pairs, the complex conjugate pair $(L, \bar{L})$ and the holomorphic pair $(L, \widetilde{L})$ determine two different null vector direction fields at $\mathfrak{I}^{+}$, the real vector field, $l^{* a}$, and the complex field, $l_{C}^{* a}$, via the relations

$$
l^{a} \rightarrow l^{* a}=l^{a}-\frac{\bar{L}}{r} m^{a}-\frac{L}{r} \bar{m}^{a}+O\left(r^{-2}\right),
$$

and

$$
l^{a} \rightarrow l_{C}^{* a}=l^{a}-\frac{\widetilde{L}}{r} m^{a}-\frac{L}{r} \bar{m}^{a}+O\left(r^{-2}\right) .
$$


Both generate, in the spacetime interior, shear-free null geodesic congruences: the first is a real twisting shear-free congruence while the latter is a complex twist-free congruence that consists of the light-cones from the world line, $z^{a}=\xi^{a}(\tau)$, i.e., they focus on $\xi^{a}(\tau)$. It is this fact that they focus on the world line, $z^{a}=\xi^{a}(\tau)$ that is of most relevance to us.

The twist of the real congruence, $\Sigma\left(u_{B}, \zeta, \bar{\zeta}\right)$, which comes from the complex divergence,

$$
\begin{aligned}
\rho & =-\frac{1}{r+i \Sigma} \\
2 i \Sigma & =ð \bar{L}+L(\bar{L})^{\cdot}-\overline{\mathrm{\partial}} L-\bar{L} \dot{L} . \\
& =\left(\xi^{a}(\tau)-\bar{\xi}^{a}(\bar{\tau})\right)\left(n_{a}-l_{a}\right) .
\end{aligned}
$$

is proportional to the imaginary part of the complex world line and consequently we have the real structure associated with the complex world line coming from two (dual) places, the real cuts, Eq. (3.26) and the twist.

It is the complex point of view of the complex light-cones coming from the complex world line that dominates our discussion.

\subsection{Approximations}

Due to the difficulties involved in the intrinsic nonlinearities and the virtual impossibility of exactly inverting arbitrary analytic functions, it often becomes necessary to resort to approximations. The basic approximation will be to consider the complex world line $\xi^{a}(\tau)$ as being close to the straight line, $\xi_{0}^{a}(\tau)=\tau \delta_{0}^{a}$; deviations from this will be considered as first order. We retain terms up to second order, i.e., quadratic terms. Another frequently used approximation is to terminate spherical harmonic expansions after the $l=2$ terms.

It is worthwhile to discuss some of the issues related to these approximations. One important issue is how to use the gauge freedom, Eq. (3.8), $\tau \rightarrow \tau^{*}=F(\tau)$, to simplify $\xi^{a}(\tau)$ and the 'velocity vector',

$$
v^{a}(\tau)=\xi^{a \prime}(\tau) \equiv \frac{d \xi^{a}}{d \tau}
$$

A Notational issue: Given a complex analytic function (or vector) of the complex variable $\tau$, say $G(\tau)$, then $G(\tau)$ can be decomposed uniquely into two parts,

$$
G(\tau)=\mathfrak{G}_{R}(\tau)+i \mathfrak{G}_{I}(\tau)
$$

where all the coefficients in the Taylor series for $\mathfrak{G}_{R}(\tau)$ and $\mathfrak{G}_{I}(\tau)$ are real. With but a slight extension of conventional notation we refer to them as real analytic functions.

With this notation, we also write

$$
\begin{gathered}
\xi^{a}(\tau)=\xi_{R}^{a}(\tau)+i \xi_{I}^{a}(\tau) \\
v^{a}(\tau)=v_{R}^{a}(\tau)+i v_{I}^{a}(\tau) .
\end{gathered}
$$

By using the reparametrization of the world line, via $\tau^{*}=F(\tau)$, we choose $F(\tau)=\xi^{0}(\tau)$, so that (dropping the ${ }^{*}$ ) we have

$$
\begin{aligned}
& \xi^{0}(\tau)=\xi_{R}^{0}(\tau)=\tau, \quad \xi_{I}^{0}(\tau)=0 \\
& v^{0}(\tau)=v_{R}^{0}(\tau)=1, \quad v_{I}^{0}(\tau)=0
\end{aligned}
$$

Finally, from the reality condition on the $u_{\mathrm{B}}$, Eqs. (3.23), (3.26) and (3.25) yield, with $\tau=s+i \lambda$ 
and $\lambda$ treated as small,

$$
\begin{aligned}
u_{\mathrm{B}}^{(R)} & =\xi_{R}^{a}(s) \hat{l}_{a}+v_{I}^{a}(s) \hat{l}_{a} \frac{\xi_{I}^{b}(s) \hat{l}_{b}}{\xi_{R}^{c \prime}(s) \hat{l}_{c}}, \\
\lambda & =\Lambda(s, \zeta, \bar{\zeta})=-\frac{\xi_{I}^{b}(s) \hat{l}_{b}}{\xi_{R}^{c \prime}(s) \hat{l}_{c}}, \\
& =\frac{\frac{\sqrt{2}}{2} \xi_{I}^{i}(s) Y_{1 i}^{0}}{1-\frac{\sqrt{2}}{2} \xi_{R}^{i \prime}(s) Y_{1 i}^{0}} .
\end{aligned}
$$

Within this slow motion approximation scheme, we have from Eqs. (3.40) and (3.41),

$$
\begin{aligned}
u_{\mathrm{ret}}^{(R)} & =\sqrt{2} u_{\mathrm{B}}^{(R)}=s-\frac{1}{\sqrt{2}} \xi_{R}^{i}(s) Y_{1 i}^{0}+2 v_{I}^{a}(s) \hat{l}_{a} \xi_{I}^{b}(s) \hat{l}_{b}, \\
\lambda & \approx \frac{\sqrt{2}}{2} \xi_{I}^{i}(s) Y_{1 i}^{0}\left(1-\frac{\sqrt{2}}{2} v_{R}^{j}(s) Y_{1 j}^{0}\right),
\end{aligned}
$$

or, to first order, which is all that is needed,

$$
\lambda=\frac{\sqrt{2}}{2} \xi_{I}^{i}(s) Y_{1 i}^{0} .
$$

We then have, to linear order,

$$
\begin{aligned}
\tau & =s+i \frac{\sqrt{2}}{2} \xi_{I}^{i}(s) Y_{1 i}^{0}, \\
u_{\text {ret }}^{(R)} & =s-\frac{1}{\sqrt{2}} \xi_{R}^{i}(s) Y_{1 i}^{0} .
\end{aligned}
$$

\subsection{Asymptotically-vanishing Maxwell fields}

\subsubsection{A prelude}

The basic starting idea in this work is simple. It is in the generalizations and implementations where difficulties arise.

Starting in Minkowski space in a fixed given Lorentzian frame with spatial origin, the electric dipole moment $\vec{D}_{E}$ is calculated from an integral over the (localized) charge distribution. If there is a shift, $\vec{R}$, in the origin, the dipole transforms as

$$
\overrightarrow{D_{E}^{*}}=\vec{D}_{E}-q \vec{R}
$$

If $\vec{D}_{E}$ is time dependent, we obtain the center-of-charge world line by taking $\overrightarrow{D_{E}^{*}}=0$, i.e., from $\vec{R}=\vec{D}_{E} q^{-1}$. It is this idea that we want to generalize and extend to gravitational fields.

The first generalization is formal and somewhat artificial: shortly it will become quite natural. We introduce, in addition to the electric dipole moment, the magnetic dipole moment $\vec{D}_{M}$ (also obtained by an integral over the current distribution) and write

$$
\vec{D}_{\mathbb{C}}=\vec{D}_{E}+i \vec{D}_{M}
$$

By allowing the displacement $\vec{R}$ to take complex values, $\vec{R}_{\mathbb{C}}$, Eq. (6.37), can be generalized to

$$
\vec{D}_{\mathbb{C}}^{*}=\vec{D}_{\mathbb{C}}-q \vec{R}_{\mathbb{C}},
$$


so that the complex center-of-charge is given by $\overrightarrow{D_{\mathbb{C}}^{*}}=0$ or

$$
\vec{R}_{\mathbb{C}}=\vec{D}_{\mathbb{C}} q^{-1} .
$$

We emphasize that this is done in a fixed Lorentz frame and only the origin is moved. In different Lorentz frames there will be different complex centers of charge.

Later, directly from the general asymptotic Maxwell field itself (satisfying the Maxwell equations), we define the asymptotic complex dipole moment and give its transformation law, including transformations between Lorentz frames. This yields a unique complex center of charge independent of the Lorentz frame.

\subsubsection{Asymptotically-vanishing Maxwell fields: General properties}

In this section, we describe how a complex center of charge for asymptotically vanishing Maxwell fields in flat spacetime can be found by using the shear-free NGCs, constructed from solutions of the homogeneous good-cut equation, to transform certain Maxwell field components to zero. Although this serves as a good example for our later methods in asymptotically flat spacetimes, the reader may wish to skip ahead to Section 4, where we go directly to gravitational fields in a setting of greater generality.

Our first set of applications of shear-free NGCs comes from Maxwell theory in Minkowski space. We review the general theory of the behavior of asymptotically-flat or vanishing Maxwell fields assuming throughout that there is a nonvanishing total charge, $q$. As stated in Section 2, the Maxwell field is described in terms of its complex tetrad components, $\left(\phi_{0}, \phi_{1}, \phi_{2}\right)$. In a Bondi coordinate/tetrad system the asymptotic integration is relatively simple $[50,38]$ resulting in the radial behavior (the peeling theorem):

$$
\begin{aligned}
& \phi_{0}=\frac{\phi_{0}^{0}}{r^{3}}+O\left(r^{-4}\right), \\
& \phi_{1}=\frac{\phi_{1}^{0}}{r^{2}}+O\left(r^{-3}\right), \\
& \phi_{2}=\frac{\phi_{2}^{0}}{r}+O\left(r^{-2}\right),
\end{aligned}
$$

where the leading coefficients of $r,\left(\phi_{0}^{0}, \phi_{1}^{0}, \phi_{2}^{0}\right)$ satisfy the evolution equations:

$$
\begin{aligned}
\dot{\phi}_{0}^{0}+\partial \phi_{1}^{0} & =0, \\
\dot{\phi}_{1}^{0}+\partial \phi_{2}^{0} & =0 .
\end{aligned}
$$

The formal integration procedure is to take $\phi_{2}^{0}$ as an arbitrary function of $\left(u_{\mathrm{B}}, \zeta, \bar{\zeta}\right)$ (the free broadcasting data), then integrate the second, for $\phi_{1}^{0}$, with a time-independent spin-weight $s=0$ function of integration and finally integrate the first, for $\phi_{0}^{0}$. Using a slight modification of this, namely from the spherical harmonic expansion, we obtain,

$$
\begin{aligned}
& \phi_{0}^{0}=\phi_{0 i}^{0} Y_{1 i}^{1}+\phi_{0 i j}^{0} Y_{2 i j}^{1}+\ldots, \\
& \phi_{1}^{0}=q+\phi_{1 i}^{0} Y_{1 i}^{0}+\phi_{1 i j}^{0} Y_{2 i j}^{0}+\ldots, \\
& \phi_{2}^{0}=\phi_{2 i}^{0} Y_{1 i}^{-1}+\phi_{2 i j}^{0} Y_{2 i j}^{-1}+\ldots,
\end{aligned}
$$

with the harmonic coefficients related to each other by the evolution equations:

$$
\begin{aligned}
& \phi_{0}^{0}=2 q \eta^{i}\left(u_{\mathrm{ret}}\right) Y_{1 i}^{1}+Q_{\mathbb{C}}^{i j \prime} Y_{2 i j}^{1}+\ldots, \\
& \phi_{1}^{0}=q+\sqrt{2} q \eta^{i \prime}\left(u_{\mathrm{ret}}\right) Y_{1 i}^{0}+\frac{\sqrt{2}}{6} Q_{\mathbb{C}}^{i j \prime \prime} Y_{2 i j}^{0}+\ldots, \\
& \phi_{2}^{0}=-2 q \eta^{i \prime \prime}\left(u_{\mathrm{ret}}\right) Y_{1 i}^{-1}-\frac{1}{3} Q_{\mathbb{C}}^{i j \prime \prime} Y_{2 i j}^{-1}+\ldots
\end{aligned}
$$


The physical meaning of the coefficients are

$$
\begin{aligned}
q & =\text { total electric charge, } \\
q \eta^{i} & =D_{\mathbb{C}}^{i}=\text { complex (electric \& magnetic) dipole moment }=D_{E}^{i}+i D_{M}^{i}, \\
Q_{\mathbb{C}}^{i j} & =\text { complex (electric \& magnetic) quadrupole moment }
\end{aligned}
$$

etc. Recall from Section 1.1 that this electromagnetic quadrupole needs to be rescaled $\left(Q_{\mathbb{C}}^{i j} \rightarrow\right.$ $\left.2 \sqrt{2} Q_{\mathbb{C}}^{i j}\right)$ to obtain the physical quadrupole which appears in the usual expressions for Maxwell theory [43]. For later use, the complex dipole is written as $D_{\mathbb{C}}^{i}\left(u_{\text {ret }}\right)=q \eta^{i}\left(u_{\text {ret }}\right)$. Note that the $D_{\mathbb{C}}^{i}$ is defined relative to a given Bondi system. This is the analogue of a given origin for the calculations of the dipole moments of Eq. (6.37).

Later in this section it will be shown that we can find a unique complex world line, $\xi^{a}(\tau)=$ $\left(\xi^{0}, \xi^{i}\right)$, (the world line associated with a shear-free NGC), that is closely related to the $\eta^{i}\left(u_{\text {ret }}\right)$. From this complex world line we can define the intrinsic complex dipole moment, $D_{\mathcal{I C}}^{i}=q \xi^{i}(s)$.

However, we first discuss a particular Maxwell field, $F^{a b}$, where one of its eigenvectors is a tangent field to a shear-free NGC. This solution, referred to as the complex Liénard-Wiechert field is the direct generalization of the ordinary Liénard-Wiechert field. Though it is a real solution in Minkowski space, it can be thought of as arising from a complex world line in complex Minkowski space.

\subsubsection{A coordinate and tetrad system attached to a shear-free NGC}

The parametric form of the general NGC was given earlier by Eq. (3.2),

$$
x^{a}=u_{\mathrm{B}}\left(\hat{l}^{a}+\hat{n}^{a}\right)-L \overline{\hat{m}}^{a}-\bar{L} \hat{m}^{a}+\left(r^{*}-r_{0}\right) \hat{l}^{a} .
$$

The parameters $\left(u_{\mathrm{B}}, \zeta, \bar{\zeta}\right)$ labeled the individual members of the congruence while $r^{*}$ was the affine parameter along the geodesics. An alternative interpretation of the same equation is to consider it as the coordinate transformation between the coordinates, $x^{a}$ (or the Bondi coordinates) and the geodesic coordinates $\left(u_{\mathrm{B}}, r^{*}, \zeta, \bar{\zeta}\right)$. Note that while these coordinates are not Bondi coordinates, though, in the limit, at $\mathfrak{I}^{+}$, they are. The associated (geodesic) tetrad is given as a function of these geodesic coordinates, but with Minkowskian components by Eqs. (3.56). We restrict ourselves to the special case of the coordinates and tetrad associated with the $L$ from a shear-free NGC. Though we are dealing with a real shear-free twisting congruence, the congruence, as we saw, is generated by a complex analytic world line in the complexified Minkowski space, $z^{a}=\xi^{a}(\tau)$. The complex parameter, $\tau$, must in the end be chosen so that the ' $u_{\mathrm{B}}$ ' of Eq. (3.19) is real. The Minkowski metric and the spin coefficients associated with this geodesic system can be calculated [40] in the $\left(u_{\mathrm{B}}, r^{*}, \zeta, \bar{\zeta}\right)$ frame. Unfortunately, it must be stated parametrically, since the $\tau$ explicitly appears via the $\xi^{a}(\tau)$ and can not be directly eliminated. (An alternate choice of these geodesic coordinates is to use the $\tau$ instead of the $u_{\mathrm{B}}$. Unfortunately, this leads to an analytic flat metric on the complexified Minkowski space, where the real spacetime is hard to find.)

The use and insight given by this coordinate/tetrad system is illustrated by its application to a special class of Maxwell fields. We consider, as mentioned earlier, the Maxwell field where one of its principle null vectors, $l^{* a}$, (an eigenvector of the Maxwell tensor, $F_{a b} l^{* a}=\lambda l_{b}^{*}$ ), is a tangent vector of a shear-free NGC. Thus, it depends on the choice of the complex world line and is therefore referred to as the complex Liénard-Wiechert field. (If the world line was real it would lead to the ordinary Liénard-Wiechert field.) We emphasize that though the source can formally be thought of as a charge moving on the complex world line, the Maxwell field is a real field on real Minkowski space. It will have a real (distributional) source at the caustics of the congruence. Physically, its behavior is very similar to real Liénard-Wiechert fields, the essential difference is that the electric 
dipole is now replaced by the combined electric and magnetic dipoles. The imaginary part of the world line determines the magnetic dipole moment.

In the spin-coefficient version of the Maxwell equations, using the geodesic tetrad, the choice of $l^{* a}$ as the principle null vector 'congruence' is just the statement that

$$
\phi_{0}^{*}=F_{a b} l^{* a} m^{* b}=0 .
$$

This allows a very simple exact integration of the remaining Maxwell components [50].

\subsubsection{Complex Liénard-Wiechert Maxwell field}

The present section, included as an illustration of the general ideas and constructions in this work, is rather technical and complicated and can be omitted without loss of continuity.

The complex Liénard-Wiechert fields (which we again emphasize are real Maxwell fields) are formally given by the (geodesic) tetrad components of the Maxwell tensor in the null geodesic coordinate system $\left(u_{\mathrm{B}}, r^{*}, \zeta, \bar{\zeta}\right)$, Eq. (3.56). As the detailed calculations are long [50] and take us too far afield, we only give an outline here. The integration of the radial Maxwell equations leads to the asymptotic behavior,

$$
\begin{aligned}
\phi_{0}^{*} & =0, \\
\phi_{1}^{*} & =\rho^{2} \phi_{1}^{* 0}, \\
\phi_{2}^{*} & =\rho \phi_{2}^{* 0}+O\left(\rho^{2}\right),
\end{aligned}
$$

with

$$
\begin{aligned}
\rho & =-\left(r^{*}+i \Sigma\right)^{-1}, \\
2 i \Sigma & =ð \bar{L}+L \dot{\bar{L}}-\bar{\partial} L-\bar{L} \dot{L} .
\end{aligned}
$$

The $O\left(\rho^{2}\right)$ expression is known in terms of $\left(\phi_{1}^{* 0}, \phi_{2}^{* 0}\right)$. The function $L\left(u_{\mathrm{B}}, \zeta, \bar{\zeta}\right)$ is given by

$$
\begin{aligned}
L\left(u_{\mathrm{B}}, \zeta, \bar{\zeta}\right) & =\partial_{(\tau)} G(\tau, \zeta, \bar{\zeta}), \\
u_{\mathrm{B}} & =G(\tau, \zeta, \bar{\zeta})=\xi^{a}(\tau) \hat{l}_{a}(\zeta, \bar{\zeta}),
\end{aligned}
$$

with $\xi^{a}(\tau)$ an arbitrary complex world line that determines the shear-free congruence whose tangent vectors are the Maxwell field eigenvectors.

Remark 8. In this case of the complex Liénard-Wiechert Maxwell field, the $\xi^{a}$ determines the intrinsic center-of-charge world line, rather than the relative center-of-charge line.

The remaining unknowns, $\phi_{1}^{* 0}, \phi_{2}^{* 0}$, are determined by the last of the Maxwell equations,

$$
\begin{aligned}
\precsim \phi_{1}^{* 0}+L \dot{\phi}_{1}^{* 0}+2 \dot{L} \phi_{1}^{* 0} & =0, \\
\precsim \phi_{2}^{* 0}+L \dot{\phi}_{2}^{* 0}+\dot{L} \phi_{2}^{* 0} & =\dot{\phi}_{1}^{* 0},
\end{aligned}
$$

which have been obtained from Eqs. (2.55) and (2.56) via the null rotation between the Bondi and geodesic tetrads and the associated Maxwell field transformation, namely,

$$
\begin{aligned}
l^{a} & \rightarrow l^{* a}=l^{a}-\frac{\bar{L}}{r} m^{a}-\frac{L}{r} \bar{m}^{a}+O\left(r^{*-2}\right), \\
m^{a} \rightarrow m^{* a} & =m^{a}-\frac{L}{r} n^{a}, \\
n^{a} \rightarrow n^{* a} & =n^{a},
\end{aligned}
$$


with

$$
\begin{aligned}
& \phi_{0}^{* 0}=0=\phi_{0}^{0}-2 L \phi_{1}^{0}+L^{2} \phi_{2}^{0}, \\
& \phi_{1}^{* 0}=\phi_{1}^{0}-L \phi_{2}^{0}, \\
& \phi_{2}^{* 0}=\phi_{2}^{0} .
\end{aligned}
$$

These remaining equations depend only on $L\left(u_{\mathrm{B}}, \zeta, \bar{\zeta}\right)$, which, in turn, is determined by $\xi^{a}(\tau)$. In other words, the solution is driven by the complex line, $\xi^{a}(\tau)$. As they now stand, Eqs. (3.61) appear to be difficult to solve, partially due to the implicit description of the $L\left(u_{\mathrm{B}}, \zeta, \bar{\zeta}\right)$.

Actually they are easily solved when the independent variables are changed, via Eq. (3.15), from $\left(u_{\mathrm{B}}, \zeta, \bar{\zeta}\right)$ to the complex $(\tau, \zeta, \bar{\zeta})$. They become, after a bit of work,

$$
\begin{aligned}
\varlimsup_{(\tau)}\left(V^{2} \phi_{1}^{0}\right) & =0, \\
\varlimsup_{(\tau)}\left(V \phi_{2}^{0}\right) & =\phi_{1}^{0 \prime}, \\
V & =\xi^{a \prime}(\tau) \hat{l}_{a}(\zeta, \bar{\zeta}),
\end{aligned}
$$

with the solution

$$
\begin{aligned}
\phi_{1}^{* 0} & =\frac{q}{2} V^{-2}, \\
\phi_{2}^{* 0} & =\frac{q}{2} V^{-1} \bar{\partial}_{(\tau)}\left(V^{-1} \partial_{\tau} V\right) .
\end{aligned}
$$

$q$ being the Coulomb charge.

Though we now have the exact solution, unfortunately it is in complex coordinates where virtually every term depends on the complex variable $\tau$, via $\xi^{a}(\tau)$. This is a severe impediment to a full description and understanding of the solution in the real Minkowski space.

In order to understand its asymptotic behavior and physical content, one must transform it, via Eqs. (3.62)-(3.67), back to a Bondi coordinate/tetrad system. This can only be done by approximations. After a lengthy calculation [50], we find the Bondi peeling behavior

$$
\begin{aligned}
& \phi_{0}=r^{-3} \phi_{0}^{0}+O\left(r^{-4}\right), \\
& \phi_{1}=r^{-2} \phi_{1}^{0}+O\left(r^{-3}\right), \\
& \phi_{2}=r^{-1} \phi_{2}^{0}+O\left(r^{-2}\right),
\end{aligned}
$$

with

$$
\begin{aligned}
\phi_{0}^{0} & =q\left(L V^{-2}+\frac{1}{2} L^{2} V^{-1} \bar{ळ}_{(\tau)}\left[V^{-1} V^{\prime}\right]\right), \\
\phi_{1}^{0} & =\frac{q}{2 V^{2}}\left(1+L V \bar{ळ}_{(\tau)}\left[V^{-1} V^{\prime}\right]\right), \\
\phi_{2}^{0} & =-\frac{q}{2} V^{-1} \bar{\delta}_{(\tau)}\left(V^{-1} V^{\prime}\right), \\
V & =\xi^{a \prime} \hat{l}_{a}(\zeta, \bar{\zeta}) .
\end{aligned}
$$

Next, treating the world line, as discussed earlier, as a small deviation from the straight line, $\xi^{a}(\tau)=\tau \delta_{0}^{a}$, i.e., by

$$
\begin{aligned}
& \xi^{a}(\tau)=\left(\tau, \xi^{i}(\tau)\right), \\
& \xi^{i}(\tau) \ll 1 .
\end{aligned}
$$


The GCF and its inverse (see Section 6) are given, to first order, by

$$
\begin{aligned}
u_{\mathrm{ret}} & =\sqrt{2} u_{\mathrm{B}}=\sqrt{2} G=\tau-\frac{\sqrt{2}}{2} \xi^{i}(\tau) Y_{1 i}^{0}(\zeta, \bar{\zeta}), \\
\tau & =u_{\mathrm{ret}}+\frac{\sqrt{2}}{2} \xi^{i}\left(u_{\mathrm{ret}}\right) Y_{1 i}^{0}(\zeta, \bar{\zeta}) .
\end{aligned}
$$

Again to first order, Eqs. (3.73), (3.74) and (3.75) yield

$$
\begin{aligned}
& \phi_{0}^{0}=2 q \xi^{i}\left(u_{\text {ret }}\right) Y_{1 i}^{1}, \\
& \phi_{1}^{0}=q+\sqrt{2} q \xi^{i \prime}\left(u_{\text {ret }}\right) Y_{1 i}^{0}, \\
& \phi_{2}^{0}=-2 q \xi^{i \prime \prime}\left(u_{\text {ret }}\right) Y_{1 i}^{-1},
\end{aligned}
$$

the known electromagnetic dipole field, with a Coulomb charge, $q$. One then has the physical interpretation of $q \xi^{i}\left(u_{\mathrm{ret}}\right)$ as the complex dipole moment; (the electric plus ' $i$ ' times magnetic dipole) and $\xi^{i}\left(u_{\text {ret }}\right)$ is the complex center of charge, the real part being the ordinary center of charge, while the imaginary part is the 'imaginary' magnetic center of charge. This simple relationship between the Bondi form of the complex dipole moment, $q \xi^{i}\left(u_{\text {ret }}\right)$, and the intrinsic complex center of charge, $\xi^{i}(\tau)$, is true only at linear order. The second-order relationship is given later.

Reversing the issue, if we had instead started with an exact complex Liénard-Wiechert field but now given in a Bondi coordinate/tetrad system and performed on it the transformations, Eqs. (2.10) and (3.65) to the geodesic system, it would have resulted in

$$
\phi_{0}^{*}=0 .
$$

This example was intended to show how physical meaning could be attached to the complex world line associated with a shear-free NGC. In this case and later in the case of asymptotically-flat spacetimes, when the GCF is singled out by either the Maxwell field or the gravitational field, it will be referred to it as a UCF. For either of the two cases, a flat-space asymptotically-vanishing Maxwell field (with nonvanishing total charge) and for a vacuum asymptotically-flat spacetime, there will be a unique UCF. In the case of the Einstein-Maxwell fields there will, in general, be two UCFs: one for each field.

\subsubsection{Asymptotically vanishing Maxwell fields \& shear-free NGCs}

We return now to the general asymptotically-vanishing Maxwell field, Eqs. (3.48) and (3.51), and its transformation behavior under the null rotation around $n^{a}$,

$$
\begin{aligned}
l^{a} \rightarrow l^{* a} & =l^{a}-\frac{\bar{L}}{r} m^{a}-\frac{L}{r} \bar{m}^{a}+0\left(r^{-2}\right), \\
m^{a} \rightarrow m^{* a} & =m^{a}-\frac{L}{r} n^{a}+0\left(r^{-2}\right), \\
n^{a} \rightarrow n^{* a} & =n^{a},
\end{aligned}
$$

with $L\left(u_{\mathrm{B}}, \zeta, \bar{\zeta}\right)=\xi^{a}(\tau) \hat{m}_{a}$, being one of our shear-free angle fields defined by a world line, $z^{a}=$ $\xi^{a}(\tau)$ and cut function $u_{\mathrm{B}}=\xi^{a}(\tau) \hat{l}_{a}(\zeta, \bar{\zeta})$. The leading components of the Maxwell fields transform as

$$
\begin{aligned}
& \phi_{0}^{* 0}=\phi_{0}^{0}-2 L \phi_{1}^{0}+L^{2} \phi_{2}^{0}, \\
& \phi_{1}^{* 0}=\phi_{1}^{0}-L \phi_{2}^{0}, \\
& \phi_{2}^{* 0}=\phi_{2}^{0} .
\end{aligned}
$$


The 'picture' to adopt is that the new $\phi^{*}$ s are now given in a tetrad defined by the complex light cone (or generalized light cone) with origin on the complex world line. (This is obviously formal and perhaps physically nonsensical, but mathematically quite sound, as the shear-free congruence can be thought of as having its origin on the complex line, $\xi^{a}(\tau)$.) From the physical identifications of charge, dipole moments, etc., of Eq. (3.54), we can obtain the transformation law of these physical quantities. In particular, the $l=1$ harmonic of $\phi_{0}^{0}$, or, equivalently, the complex dipole, transforms as

$$
\phi_{0 i}^{0 *}=\phi_{0 i}^{0}-\left.2\left(L \phi_{1}^{0}\right)\right|_{i}+\left.\left(L^{2} \phi_{2}^{0}\right)\right|_{i},
$$

where the notation $\left.W\right|_{i}$ means extract only the $l=1$ harmonic from a Clebsch-Gordon expansion of $W$. A subtlety and difficulty of this extraction process is here clarified.

\section{The (non-)uniqueness of spherical harmonic expansions}

An important observation, obvious but easily overlooked, concerning the spherical harmonic expansions is that, in a certain sense, they lack uniqueness. As this issue is significant, its clarification is important.

Assume that we have a particular spin- $s$ function on $\mathfrak{I}^{+}$, say, $\eta_{(s)}\left(u_{\mathrm{B}}, \zeta, \bar{\zeta}\right)$, given in a specific Bondi coordinate system, $\left(u_{\mathrm{B}}, \zeta, \bar{\zeta}\right)$, that has a harmonic expansion given, for constant $u_{\mathrm{B}}$, by

$$
\eta_{(s)}\left(u_{\mathrm{B}}, \zeta, \bar{\zeta}\right)=\sum_{l,(i j k \ldots)} \eta_{(s)}^{l,(i j k \ldots)}\left(u_{\mathrm{B}}\right) Y_{l,(i j k \ldots)}^{(s)}
$$

If exactly the same function was given on different cuts or slices, say,

$$
u_{\mathrm{B}}=G(\tau, \zeta, \bar{\zeta}),
$$

with

$$
\eta_{(s)}^{*}(\tau, \zeta, \bar{\zeta})=\eta_{(s)}(G(\tau, \zeta, \bar{\zeta}), \zeta, \bar{\zeta})
$$

the harmonic expansion at constant $\tau$ would be different. The new coefficients are extracted by the two-sphere integral taken at constant $\tau$ :

$$
\eta_{(s)}^{* l,(i j k \ldots)}(\tau)=\int_{S^{2}} \eta_{(s)}^{*}(\tau, \zeta, \bar{\zeta}) \bar{Y}_{l,(i j k \ldots)}^{(s)} d S .
$$

It is in this rather obvious sense that the expansions are not unique.

The transformation, Eq. (3.84), and harmonic extraction implemented by first replacing the $u_{B}$ in all the terms of all $\phi_{0}^{0}, \phi_{1}^{0}, \phi_{2}^{0}$, by $u_{\mathrm{B}}=\xi^{a}(\tau) \hat{l}_{a}(\zeta, \bar{\zeta}) \equiv \frac{\sqrt{2} \tau}{2}-\frac{1}{2} \xi^{i} Y_{1 i}^{0}$, yields $\phi_{0 i}^{0 *}(\tau)$ with a functional form [8],

$$
\phi_{0 i}^{0 *}(\tau)=\Gamma_{i}\left(\phi_{0}^{0}, \phi_{1}^{0}, \phi_{2}^{0}, \xi^{a}(\tau)\right)=\oint_{S^{2}}\left[\phi_{0}^{0}-\left(2 c L \phi_{1}^{0}-c^{2} L^{2} \phi_{2}^{0}\right)\right] Y_{1 i}^{-1} d S
$$

is decidedly nontensorial: in fact it is very nonlocal and nonlinear.

Though it is clear that extracting $\phi_{0 i}^{0 *}(\tau)$ with this relationship is available in principle, in practice it is impossible to do it exactly and all examples are done with approximations: essentially using slow motion for the complex world line.

Remark 9. If by some accident the Maxwell field was a complex Liénard-Wiechert field, a world line $\xi^{a}(\tau)$ could be chosen so that from the associated complex null cones we would have $\phi_{0}^{* 0}=0$. However, though this cannot be done in general, the $l=1$ harmonics of $\phi_{0}^{* 0}$ can be made to vanish by the appropriate choice of the $\xi^{a}(\tau)$. This is the means by which a unique world line is chosen. 


\subsubsection{The complex center of charge}

The complex center of charge is defined by the vanishing of the complex dipole moment $\phi_{0 i}^{0 *}(\tau)$; in other words,

$$
\Gamma_{i}\left(\phi_{0}^{0}, \phi_{1}^{0}, \phi_{2}^{0}, \xi^{a}\right)=0
$$

determines three components of the (up to now) arbitrary complex world line, $\xi^{a}(\tau)$; the fourth component can be taken as $\tau$. In practice we do this only up to second order with the use of only the $(l=0,1,2)$ harmonics. The approximation we are using is to consider the charge $q$ as zeroth order and the dipole moments and the spatial part of the complex world line as first order.

From Eq. (3.84),

$$
\phi_{0 i}^{0 *}=\Gamma_{i} \approx \phi_{0 i}^{0}-\left.2 L \phi_{1}^{0}\right|_{i}=0
$$

with the identifications, Eq. (3.54), for $q$ and $D_{\mathbb{C}}$, we have to first order (with $\sqrt{2} u_{B}=u_{r e t} \approx \tau$ ),

$$
D_{\mathbb{C}}^{i}\left(u_{\text {ret }}\right)=q \eta^{i}\left(u_{\text {ret }}\right)=q \xi^{i}\left(u_{\text {ret }}\right) .
$$

This is exactly the same result as we obtained earlier in Eq. (3.47), via the charge and current distributions in a fixed Lorentz frame.

Carrying this calculation [50] to second order, we find the second-order complex center of charge and the relationship between the intrinsic complex dipole, $D_{\mathcal{I}: \mathbb{C}}^{i}$, and the complex dipole, $D_{\mathbb{C}}^{i}$,

$$
\begin{aligned}
D_{\mathcal{I}: \mathbb{C}}^{i} & =q \xi^{i}(s), \quad D_{\mathbb{C}}^{i}=q \eta^{i}(s), \\
\xi^{k} & =\eta^{k}-\frac{i}{2} \eta^{i} \eta^{j \prime} \epsilon_{i j k}-\frac{\sqrt{2}}{10} q^{-1} Q_{\mathbb{C}}^{i k \prime \prime} \xi^{i}, \\
\eta^{k} & =\xi^{k}+i \frac{1}{2} \xi^{i} \xi^{j \prime} \epsilon_{i j k}+\frac{\sqrt{2}}{10} q^{-1} Q_{\mathbb{C}}^{i k \prime \prime} \xi^{i}
\end{aligned}
$$

The GCF,

$$
u_{\mathrm{B}}=X(\tau, \zeta, \tilde{\zeta})=\xi^{a}(\tau) \hat{l}_{a}(\zeta, \tilde{\zeta}),
$$

with this uniquely determined world line is referred to as the Maxwell UCF.

In Section 5, these ideas are applied to GR, with the complex electric and magnetic dipoles being replaced by the complex combination of the mass dipole and the angular momentum. 


\section{The Good-Cut Equation and $\mathcal{H}$-Space}

In Section 3, we discussed NGCs in Minkowski spacetime that were shear-free. In this section we consider asymptotically shear-free NGCs in asymptotically-flat spacetimes. That is to say, we consider NGCs that have nonvanishing shear in the interior of the spacetime but where, as null infinity is approached, the shear vanishes. Whereas fully shear-free NGCs almost never occur in general asymptotically flat spacetimes, asymptotically shear-free congruences always exist. The case of algebraically-special spacetimes is the exception; they do allow one or two shear-free congruences.

We begin by reviewing the shear-free condition and follow with its generalization to the asymptotically shear-free case. From this we derive the generalization of the homogeneous good-cut equation to the inhomogeneous good-cut equation. Almost all the properties of the shear-free and asymptotically shear-free NGCs come from the study of these equations and virtually all the attributes of shear-free congruences are shared by the asymptotically shear-free congruences. It is from the use of these shared attributes that we will be able to extract physical identifications and information (e.g., complex center of mass/charge, Bondi mass, linear and angular momentum, equations of motion, etc.) from the asymptotic gravitational fields.

Though again the use of the formal complexification of $\mathfrak{I}^{+}$, i.e., $\mathfrak{I}_{\mathbb{C}}^{+}$, is essential for our analysis, it is the extraction of the real structures that is important.

\subsection{Asymptotically shear-free NGCs and the good-cut equation}

We saw in Section 3 that shear-free NGCs in Minkowski space could be constructed by looking at their properties near $\mathfrak{I}^{+}$, in one of two equivalent ways. The first was via the stereographic angle field, $L\left(u_{\mathrm{B}}, \zeta, \bar{\zeta}\right)$, which gives the directions that null rays make at their intersection with $\mathfrak{I}^{+}$. The condition for the congruence to be shear-free was that $L$ must satisfy

$$
\varlimsup_{\left(u_{\mathrm{B}}\right)} L+L \dot{L}=0 .
$$

We required solutions that were all nonsingular (regular) on the $(\zeta, \bar{\zeta})$ sphere. (This equation has in the past most often been solved via twistor methods [31].)

The second was via the complex cut function, $u_{\mathrm{B}}=G(\tau, \zeta, \tilde{\zeta})$, that satisfied

$$
\check{\partial}_{(\tau)}^{2} G=0 .
$$

The regular solutions were easily given by

$$
u_{\mathrm{B}}=G(\tau, \zeta, \tilde{\zeta})=\xi^{a}(\tau) \hat{l}_{a}(\zeta, \tilde{\zeta})
$$

with inverse function,

$$
\tau=T\left(u_{\mathrm{B}}, \zeta, \tilde{\zeta}\right) .
$$

They determined the $L\left(u_{\mathrm{B}}, \zeta, \bar{\zeta}\right)$ that satisfies Eq. (4.1) by the parametric relations

$$
\begin{aligned}
L\left(u_{\mathrm{B}}, \zeta, \bar{\zeta}\right) & =\partial_{(\tau)} G(\tau, \zeta, \tilde{\zeta}), \\
u_{\mathrm{B}} & =\xi^{a}(\tau) \hat{l}_{a}(\zeta, \tilde{\zeta}),
\end{aligned}
$$

or by

$$
L\left(u_{\mathrm{B}}, \zeta, \bar{\zeta}\right)=\left.\partial_{(\tau)} G(\tau, \zeta, \bar{\zeta})\right|_{\tau=T\left(u_{\mathrm{B}}, \zeta, \tilde{\zeta}\right)}
$$

where $\xi^{a}(\tau)$ was an arbitrary complex world line in complex Minkowski space.

It is this pair of equations, (4.1) and (4.2), that will now be generalized to asymptotically-flat spacetimes. 
In Section 2, we saw that the asymptotic shear of the (null geodesic) tangent vector fields, $l^{a}$, of the out-going Bondi null surfaces was given by the free data (the Bondi shear) $\sigma^{0}\left(u_{\mathrm{B}}, \zeta, \bar{\zeta}\right)$. If, near $\mathfrak{I}^{+}$, a second NGC, with tangent vector $l^{* a}$, is chosen and then described by the null rotation from $l^{a}$ to $l^{* a}$ around $n^{a}$ by

$$
\begin{aligned}
l^{* a} & =l^{a}+b \bar{m}^{a}+\bar{b} m^{a}+b \bar{b} n^{a}, \\
m^{* a} & =m^{a}+b n^{a}, \\
n^{* a} & =n^{a}, \\
b & =-L / r+O\left(r^{-2}\right),
\end{aligned}
$$

with $L\left(u_{\mathrm{B}}, \zeta, \bar{\zeta}\right)$ an arbitrary stereographic angle field, then the asymptotic Weyl components transform as

$$
\begin{aligned}
& \psi_{0}^{* 0}=\psi_{0}^{0}-4 L \psi_{1}^{0}+6 L^{2} \psi_{2}^{0}-4 L^{3} \psi_{3}^{0}+L^{4} \psi_{4}^{0}, \\
& \psi_{1}^{* 0}=\psi_{1}^{0}-3 L \psi_{2}^{0}+3 L^{2} \psi_{3}^{0}-L^{3} \psi_{4}^{0}, \\
& \psi_{2}^{* 0}=\psi_{2}^{0}-2 L \psi_{3}^{0}+L^{2} \psi_{4}^{0}, \\
& \psi_{3}^{* 0}=\psi_{3}^{0}-L \psi_{4}^{0}, \\
& \psi_{4}^{* 0}=\psi_{4}^{0},
\end{aligned}
$$

and the (new) asymptotic shear of the null vector field $l^{* a}$ is given by [12, 39]

$$
\sigma^{0 *}=\sigma^{0}-\partial_{\left(u_{\mathrm{B}}\right)} L-L \dot{L} .
$$

By requiring that the new congruence be asymptotically shear-free, i.e., $\sigma^{0 *}=0$, we obtain the generalization of Eq. (4.1) for the determination of $L\left(u_{\mathrm{B}}, \zeta, \bar{\zeta}\right)$, namely,

$$
\widetilde{\partial}_{\left(u_{\mathrm{B}}\right)} L+L \dot{L}=\sigma^{0}\left(u_{\mathrm{B}}, \zeta, \bar{\zeta}\right) .
$$

To solve this equation we again complexify $\mathfrak{I}^{+}$to $\mathfrak{I}_{\mathbb{C}}^{+}$by freeing $\bar{\zeta}$ to $\tilde{\zeta}$ and allowing $u_{\mathrm{B}}$ to take complex values close to the real.

Again we introduce the complex potential $\tau=T\left(u_{\mathrm{B}}, \zeta, \tilde{\zeta}\right)$ that is related to $L$ by

$$
\mathrm{\partial}_{\left(u_{\mathrm{B}}\right)} T+L \dot{T}=0,
$$

with its inversion,

$$
u_{\mathrm{B}}=G(\tau, \zeta, \tilde{\zeta}) .
$$

Eq. (4.12) becomes, after the change in the independent variable, $u_{\mathrm{B}} \Rightarrow \tau=T\left(u_{\mathrm{B}}, \zeta, \bar{\zeta}\right)$, and implicit differentiation (see Section 3.1 for the identical details),

$$
\check{\partial}_{(\tau)}^{2} G=\sigma^{0}(G, \zeta, \tilde{\zeta}) .
$$

This, the inhomogeneous good-cut equation, is the generalization of Eq. (4.2).

In Section 4.2, we will discuss how to construct solutions of Eq. (4.15) of the form, $u_{\mathrm{B}}=$ $G(\tau, \zeta, \tilde{\zeta})$; however, assuming we have such a solution, it determines the angle field $L\left(u_{\mathrm{B}}, \zeta, \bar{\zeta}\right)$ by the parametric relations

$$
\begin{aligned}
L\left(u_{\mathrm{B}}, \zeta, \tilde{\zeta}\right) & =\partial_{(\tau)} G, \\
u_{\mathrm{B}} & =G(\tau, \zeta, \tilde{\zeta}) .
\end{aligned}
$$

We now turn to these solutions and their properties. 


\section{$4.2 \mathcal{H}$-space and the good-cut equation}

Eq. (4.15), written in earlier literature as

$$
\check{\partial}^{2} Z=\sigma^{0}(Z, \zeta, \tilde{\zeta}),
$$

is a well-known and well-studied partial differential equation, often referred to as the "goodcut equation" [31, 32]. For sufficiently regular $\sigma^{0}\left(u_{\mathrm{B}}, \zeta, \bar{\zeta}\right)$ (which is assumed here) it has been proven [32] that the solutions are determined by points in a complex four-dimensional space, $z^{a}$, referred to as $\mathcal{H}$-space, i.e., solutions are given as

$$
u_{\mathrm{B}}=Z\left(z^{a}, \zeta, \tilde{\zeta}\right) .
$$

Later in this section, by choosing an arbitrary complex analytic world line in $\mathcal{H}$-space, $z^{a}=$ $\xi^{a}(\tau)$, we describe how to construct the shear-free angle field, $L\left(u_{\mathrm{B}}, \zeta, \tilde{\zeta}\right)$. First, however, we discuss properties and the origin of Eq. (4.18).

Roughly or intuitively one can see how the four complex parameters enter the solution from the following argument. We can write Eq. (4.17) as the integral equation

$$
Z=z^{a} \hat{l}_{a}(\zeta, \tilde{\zeta})+\oint \sigma^{0}(Z, \eta, \tilde{\eta}) K_{0,-2}^{+}(\eta, \tilde{\eta}, \zeta, \tilde{\zeta}) d S_{\eta}
$$

with

$$
\begin{aligned}
K_{0,-2}^{+}(\zeta, \tilde{\zeta}, \eta, \tilde{\eta}) & \equiv-\frac{1}{4 \pi} \frac{(1+\tilde{\zeta} \eta)^{2}(\eta-\zeta)}{(1+\zeta \tilde{\zeta})(1+\eta \tilde{\eta})(\tilde{\eta}-\tilde{\zeta})}, \\
d S_{\eta} & =4 i \frac{d \eta \wedge d \tilde{\eta}}{(1+\eta \tilde{\eta})^{2}},
\end{aligned}
$$

where $z^{a} \hat{l}_{a}(\zeta, \tilde{\zeta})$ is the kernel of the $\check{\partial}^{2}$ operator (the solution to the homogeneous good-cut equation) and $K_{0,-2}^{+}(\zeta, \tilde{\zeta}, \eta, \tilde{\eta})$ is the Green's function for the $\widetilde{\partial}^{2}$ operator [36]. By iterating this equation, with the kernel being the zeroth iterate, i.e.,

$$
\begin{aligned}
Z_{n}(\zeta, \tilde{\zeta}) & =z^{a} \hat{l}_{a}(\zeta, \tilde{\zeta})+\int_{S^{2}} K_{0,-2}^{+}(\zeta, \tilde{\zeta}, \eta, \tilde{\eta}) \sigma\left(Z_{n-1}, \eta, \tilde{\eta}\right) d S_{\eta}, \\
Z_{0}(\zeta, \tilde{\zeta}) & =z^{a} \hat{l}_{a}(\zeta, \tilde{\zeta}),
\end{aligned}
$$

one easily sees how the four $z^{a}$ enter the solution. Basically, the $z^{a}$ come from the solution to the homogeneous equation.

It should be noted again that the $z^{a} \hat{l}_{a}(\zeta, \tilde{\zeta})$ is composed of the $l=(0,1)$ harmonics,

$$
z^{a} \hat{l}_{a}(\zeta, \tilde{\zeta})=\frac{1}{\sqrt{2}} z^{0}-\frac{1}{2} z^{i} Y_{1 i}^{0}(\zeta, \tilde{\zeta})
$$

Furthermore, the integral term does not contribute to these lowest harmonics. This means that solutions can be written

$$
u_{\mathrm{B}}=Z\left(z^{a}, \zeta, \tilde{\zeta}\right) \equiv z^{a} \hat{l}_{a}(\zeta, \tilde{\zeta})+Z_{l \geq 2}\left(z^{a}, \zeta, \tilde{\zeta}\right),
$$

with $Z_{l \geq 2}$ containing spherical harmonics $l=2$ and higher.

We note that using this form of the solution implies that we have set stringent coordinate conditions on the $\mathcal{H}$-space by requiring that the first four spherical harmonic coefficients be the four $\mathcal{H}$-space coordinates. Arbitrary coordinates would just mean that these four coefficients were arbitrary functions of other coordinates. How these special coordinates change under the BMS group is discussed later. 
Remark 10. It is of considerable interest that on $\mathcal{H}$-space there is a natural quadratic complex metric - as constructed in Appendix D - that is given by the surprising relationship [49, 32]

$$
\begin{aligned}
d s_{(\mathcal{H})}^{2} & =g_{(\mathcal{H}) a b} d z^{a} d z^{b} \equiv\left(\frac{1}{8 \pi} \int_{S^{2}} \frac{d S}{(d Z)^{2}}\right)^{-1} \\
d Z & \equiv \nabla_{a} Z d z^{a} \\
d S & =4 i \frac{d \zeta \wedge d \tilde{\zeta}}{(1+\zeta \tilde{\zeta})^{2}} .
\end{aligned}
$$

Remarkably this turns out to be a Ricci-flat metric with a nonvanishing anti-self-dual Weyl tensor and vanishing self-dual Weyl tenor, i.e., it is intrinsically a complex anti-self-dual vacuum metric. For vanishing Bondi shear, $\mathcal{H}$-space reduces to complex Minkowski space (i.e., $\left.\left.g_{(\mathcal{H}) a b}\right|_{\sigma^{0}=0}=\eta_{a b}\right)$.

\subsubsection{Solutions to the shear-free equation}

Returning to the issue of the solutions to the shear-free condition, i.e., Eq. $(4.12), L\left(u_{\mathrm{B}}, \zeta, \tilde{\zeta}\right)$, we see that they are easily constructed from the solutions to the good-cut equation, $u_{\mathrm{B}}=Z\left(z^{a}, \zeta, \tilde{\zeta}\right)$. By choosing an arbitrary complex world line in the $\mathcal{H}$-space, i.e.,

$$
z^{a}=\xi^{a}(\tau)
$$

we write the GCF as

$$
u_{\mathrm{B}}=G(\tau, \zeta, \tilde{\zeta}) \equiv Z\left(\xi^{a}(\tau), \zeta, \tilde{\zeta}\right)
$$

or, from Eq. (4.23),

$$
u_{\mathrm{B}}=G(\tau, \zeta, \tilde{\zeta})=\frac{1}{\sqrt{2}} \xi^{0}(\tau)-\frac{1}{2} \xi^{i}(\tau) Y_{1 i}^{0}(\zeta, \tilde{\zeta})+\xi^{i j}(\tau) Y_{2 i j}^{0}(\zeta, \tilde{\zeta})+\ldots
$$

This leads immediately, via Eqs. (4.16) and (4.29), to the parametric description of the shear-free stereographic angle field $L\left(u_{\mathrm{B}}, \zeta, \tilde{\zeta}\right)$, as well as the Bondi shear $\sigma^{0}\left(u_{\mathrm{B}}, \zeta, \tilde{\zeta}\right)$ :

$$
\begin{aligned}
u_{\mathrm{B}} & =\frac{1}{\sqrt{2}} \xi^{0}(\tau)-\frac{1}{2} \xi^{i}(\tau) Y_{1 i}^{0}(\zeta, \tilde{\zeta})+\xi^{i j}(\tau) Y_{2 i j}^{0}(\zeta, \tilde{\zeta})+\ldots, \\
L\left(u_{\mathrm{B}}, \zeta, \tilde{\zeta}\right) & =\xi^{i}(\tau) Y_{1 i}^{1}(\zeta, \tilde{\zeta})-6 \xi^{i j}(\tau) Y_{2 i j}^{1}(\zeta, \tilde{\zeta})+\ldots \\
\sigma^{0}\left(u_{\mathrm{B}}, \zeta, \tilde{\zeta}\right) & =24 \xi^{i j}(\tau) Y_{2 i j}^{2}+\ldots
\end{aligned}
$$

We denote the inverse to Eq. (4.29) by

$$
\tau=T\left(u_{\mathrm{B}}, \zeta, \tilde{\zeta}\right)
$$

and refer to the complex world line $\xi^{a}(\tau)$ as the 'virtual' source of the congruence. The asymptotic twist of the asymptotically shear-free NGC is exactly as in the flat-space case,

$$
i \Sigma=\frac{1}{2}\{ð \bar{L}+L \dot{\bar{L}}-\bar{\partial} L-\bar{L} \dot{L}\}
$$

As in the flat-space case, the derived quantity

$$
V(\tau, \zeta, \tilde{\zeta}) \equiv \partial_{\tau} G=G^{\prime}
$$

plays a large role in applications. (In the case of the Robinson-Trautman metrics [71, 41] $V$ is the basic variable for the construction of the metric.) 
Using the gauge freedom, $\tau \rightarrow \tau^{*}=\Phi(\tau)$, as in the Minkowski-space case, we impose the simple condition

$$
\xi^{0}=\tau .
$$

A Brief Summary: The description and analysis of the asymptotically shear-free NGCs in asymptotically-flat spacetimes is remarkably similar to that of the flat-space regular shear-free NGCs. We have seen that all regular shear-free NGCs in Minkowski space and asymptotically-flat spaces are generated by solutions to the good-cut equation, with each solution determined by the choice of an arbitrary complex analytic world line in complex Minkowski space or $\mathcal{H}$-space. The basic governing variables are the complex GCF, $u_{\mathrm{B}}=G(\tau, \zeta, \tilde{\zeta})$, and the stereographic angle field on $\mathfrak{I}_{\mathbb{C}}^{+}, L\left(u_{\mathrm{B}}, \zeta, \tilde{\zeta}\right)$, restricted to real $\mathfrak{I}^{+}$. In every sense, the flat-space case can be considered as a special case of the asymptotically-flat case.

In Sections 5 and 6, we will show that in every asymptotically flat spacetime a special complexworld line (along with its associated NGC and GCF) can be singled out using physical considerations. This special GCF is referred to as the (gravitational) UCF, and is denoted by

$$
u_{\mathrm{B}}=X(\tau, \zeta, \tilde{\zeta}) .
$$

\subsection{Real cuts from the complex good cuts, II}

The construction of real structures from the complex structures, i.e., finding the complex values of $\tau$ that yield real values of $u_{\mathrm{B}}$ and the associated real cuts, is virtually identical to the flatspace construction of Section 3. The real structure associated with the complex Minkowski space complex world lines is easily extended to the $\mathcal{H}$-space world lines associated with asymptotically flat spacetimes. The only difference is that we start with the GCF

$$
u_{\mathrm{B}}=G(\tau, \zeta, \bar{\zeta})=\xi^{a}(\tau) \hat{l}_{a}(\zeta, \bar{\zeta})+G_{l \geq 2}(\tau, \zeta, \bar{\zeta})
$$

rather than the flat-space

$$
u_{\mathrm{B}}=G(\tau, \zeta, \bar{\zeta})=\xi^{a}(\tau) \hat{l}_{a}(\zeta, \bar{\zeta})
$$

Again assuming that the Bondi shear is sufficiently small and the $\mathcal{H}$-space complex world line is not too far from the "real", the solution to the good-cut equation (4.15), i.e., Eq. (4.38), with $\tau=s+i \lambda$, is decomposed into real and imaginary parts,

$$
G(\tau, \zeta, \bar{\zeta})=\frac{1}{2}(G(s+i \lambda, \zeta, \bar{\zeta})+\bar{G}(s-i \lambda, \zeta, \bar{\zeta}))+\frac{1}{2}(G(s+i \lambda, \zeta, \bar{\zeta})-\bar{G}(s-i \lambda, \zeta, \bar{\zeta})) .
$$

Setting the imaginary part to zero and solving for $\lambda$ we obtain an expression of the form,

$$
\lambda=\Lambda(s, \zeta, \bar{\zeta})
$$

As in the flat case, for fixed $s=s_{0}, \Lambda$ has values on a line segment bounded between some $\lambda_{\text {min }}$ and $\lambda_{\max }$. The allowed values of $\tau$ are again on a ribbon in the $\tau$-plane (i.e., region which is topologically $\mathbb{R} \times I$ for an interval $I$ ); all values of $s$ and allowed values on the $\lambda$-line segments.

Each level curve of the function $\lambda=\Lambda\left(s_{0}, \zeta, \bar{\zeta}\right)=$ constant on the $(\zeta, \bar{\zeta})$-sphere (closed curves or isolated points) determines a specific subset of the null directions and associated null geodesics on the light-cone of the complex point $\xi^{a}\left(s_{0}+i \Lambda\left(s_{0}, \zeta, \bar{\zeta}\right)\right)$ that intersect the real $\mathfrak{I}^{+}$. These geodesics will be referred to as 'real' geodesics. As $\lambda$ moves over all allowed values of its segment, we obtain the set of $\mathcal{H}$-space points, $\xi^{a}\left(s_{0}+i \Lambda\left(s_{0}, \zeta, \bar{\zeta}\right)\right)$ and their collection of 'real' geodesics. From Eq. (4.39), these 'real' geodesics intersect $\mathfrak{I}^{+}$on the real cut

$$
u_{\mathrm{B}}^{(R)}=G_{R}\left(s_{0}+i \Lambda\left(s_{0}, \zeta, \bar{\zeta}\right), \zeta, \bar{\zeta}\right) .
$$


As $s$ varies we obtain a one-parameter family of cuts. If these cuts do not intersect with each other we say that the complex world line $\xi^{a}(\tau)$ is by definition 'timelike.' This occurs when the time component of the real part of the complex velocity vector, $v^{a}(\tau)=\mathrm{d} \xi^{a}(\tau) / \mathrm{d} \tau$, is sufficiently large.

\subsection{Summary of Real Structures}

To put the ideas of this section into perspective we collect the claims.

- In Minkowski space, the future directed light-cones emanating from a real timelike world line, $x^{a}=\xi^{a}(s)$, intersect future null infinity, $\mathfrak{I}^{+}$, on a one-parameter family of spherical non-intersecting cuts.

- The complex light-cones emanating from a timelike complex analytic curve in complex Minkowski space, $z^{a}=\xi^{a}(\tau)$ parametrized by the complex parameter $\tau=s+i \lambda$, has for each fixed value of $s$ and $\lambda$ a limited set of null geodesics that reach real $\mathfrak{I}^{+}$. However, for a ribbon in the complex $\tau$-plane (i.e., a region topologically $\mathbb{R} \times I$, with $s \in \mathbb{R}$ and $\left.\lambda \in I=\left[\lambda_{\min }, \lambda_{\max }\right]\right)$, there will be many null geodesics intersecting $\mathfrak{I}^{+}$. Such null geodesics were referred to as 'real' geodesics. More specifically, for a fixed $s$, there is a specific range of $\lambda$ values such that all the real null geodesics intersect $\mathfrak{I}^{+}$in a full cut, leading to a oneparameter family of real (distorted sphere) slicings of $\mathfrak{I}^{+}$. The ribbon is the generalization of the real world line and the slicings are the analogues of the spherical slicings. When the ribbon shrinks to a line it degenerates to the real case. We can consider the ribbon as a generalized world line and the 'real' null geodesics from a constant $s$ portion of the ribbon as a generalized light-cone.

- For the case of asymptotically flat spacetimes, the real light-cones from interior points are replaced by the virtual light-cones generated by the asymptotically shear-free NGCs. These cones emanate from a complex virtual world line $z^{a}=\xi^{a}(\tau)$ in the associated $\mathcal{H}$-space. As in the case of complex Minkowski space, there is a ribbon in the $\tau$-plane where the 'real' null geodesics originate from. The 'real' null geodesics coming from a cross-section of the strip at fixed $s$ (as in the complex Minkowski case), intersect $\mathfrak{I}^{+}$in a cut; the collection of cuts yielding a one-parameter family. The situation is exactly the same as in the complex Minkowski space case except that the spherical harmonic decomposition of these cuts is in general more complicated.

\section{Example: the (charged) Kerr metric}

Considering the Kerr or the charged Kerr metrics (or even more generally any asymptotically flat stationary metric), we have immediately that the Bondi shear $\sigma^{0}$ vanishes and hence the associated $\mathcal{H}$-space is complex Minkowski space (cf. [3, 6] and Appendix F). From the stationarity and a real origin shift and rotation, the complex world line can be put into the form

$$
\xi^{a}(\tau)=(\tau, 0,0, i a),
$$

with $a$ being the Kerr parameter. The complex cut function is then

$$
\begin{aligned}
u_{\mathrm{B}} & =\xi^{a}(\tau) \hat{l}_{a}(\zeta, \bar{\zeta}) \\
& =\frac{\tau}{\sqrt{2}}-\frac{i}{2} a Y_{1,3}^{0}(\zeta, \bar{\zeta}), \\
Y_{1,3}^{0}(\zeta, \bar{\zeta}) & =-\sqrt{2} \frac{1-\zeta \bar{\zeta}}{1+\zeta \bar{\zeta}},
\end{aligned}
$$


so that the angle fields of 3.33-3.34 are

$$
\begin{aligned}
L & =\sqrt{2} i a \frac{\bar{\zeta}}{1+\zeta \bar{\zeta}}, \\
\bar{L} & =-\sqrt{2} i a \frac{\zeta}{1+\zeta \bar{\zeta}}, \\
\widetilde{L} & =\sqrt{2} i a \frac{\zeta}{1+\zeta \bar{\zeta}} .
\end{aligned}
$$

Using $\tau=s+i \lambda$ in Eq. (4.42), the reality condition $u_{\mathrm{B}}=u_{\mathrm{B}}^{(R)}$ on the cut function is that

$$
\lambda=\Lambda(s, \zeta, \bar{\zeta})=\frac{\sqrt{2}}{2} a Y_{1,3}^{0}(\zeta, \bar{\zeta}),
$$

so that on the $\tau$-ribbon, $\lambda$ ranges between $\pm \sqrt{2}$ and the real slices from the ribbon becomes simply $u_{\mathrm{B}}=s / \sqrt{2}$.

Though we are certainly not making the claim that one can in reality 'observe' these complex world lines that arise from (asymptotically) shear-free congruences, we nevertheless claim that they can be observed in a different sense. In the next two sections our goal will be to show that, just as a complex center of charge world line in $\mathbb{M}_{\mathbb{C}}$ can be selected, so too can a complex center of mass world line be singled out in $\mathcal{H}$-space. As we will see, some surprising physical identifications arise from this program, and it is in this sense which the footprints of these complex world lines can be observed. 


\section{$5 \quad$ Simple Applications}

In this section we give four simple examples of the use of shear-free and asymptotically shearfree NGCs in GR. The first is for asymptotically-linearized perturbations off the Schwarzschild metric, while the next two are from the class of algebraically-special metrics, namely the RobinsonTrautman metric and the type II twisting metrics; the fourth is for asymptotically static/stationary metrics.

\subsection{Linearized off Schwarzschild}

As a first example, we describe how the shear-free NGCs are applied in linear perturbations off the Schwarzschild metric. The ideas used here are intended to clarify the more complicated issues in the full nonlinear asymptotic theory. We will see that these linear perturbations greatly resemble our results from Section 3.4 on the determination of the intrinsic center of charge in Maxwell theory, when there were small deviations from the Coulomb field.

We begin with the Schwarzschild spacetime, treating the Schwarzschild mass, $M_{\mathrm{Sch}} \equiv M_{\mathrm{B}}$, as a zeroth-order quantity, and integrate the linearized Bianchi identities for the linear Weyl tensor corrections. Though we could go on and find the linearized connection and metric, we stop just with the Weyl tensor. The radial behavior is given by the peeling theorem, so that we can start with the linearized asymptotic Bianchi identities, Eqs. (2.52)-(2.54).

Our main variables for the investigation are the asymptotic Weyl tensor components and the Bondi shear, $\sigma^{0}$, with their related differential equations, i.e., the asymptotic Bianchi identities, Eq. (2.52), (2.53) and (2.51). Assuming the gravitational radiation is weak, we treat $\sigma^{0}$ and $\dot{\sigma}^{0}$ as small. Keeping only linear terms in the Bianchi identities, the equations for $\psi_{1}^{0}$ and $\Psi$ (the mass aspect) become

$$
\begin{aligned}
\dot{\psi}_{1}^{0}+ð \Psi & =\check{\partial}^{3} \bar{\sigma}^{0}, \\
\dot{\Psi} & =0, \\
\Psi & =\bar{\Psi}, \\
\Psi & \equiv \psi_{2}^{0}+\check{\partial}^{2} \bar{\sigma}^{0} .
\end{aligned}
$$

The $\psi_{1}^{0}$ is small (first order), while the

$$
\Psi=\Psi^{0}+\Psi^{i} Y_{1 i}^{0}+\Psi^{i j} Y_{2 i j}^{0}+\ldots
$$

has the zeroth-order Schwarzschild mass plus first-order terms

$$
\begin{aligned}
\Psi^{0} & =-\frac{2 \sqrt{2} G}{c^{2}} M_{\mathrm{Sch}}+\delta \Psi^{0}, \\
\Psi^{i} & =-\frac{6 G}{c^{3}} P^{i} .
\end{aligned}
$$

In linear theory, the complex (mass) dipole moment,

$$
D_{\text {(grav) }}^{i}=D_{(\text {mass })}^{i}+i c^{-1} J^{i}
$$

is given [75], on a particular Bondi cut with a Bondi tetrad (up to dimensional constants), by the $l=1$ harmonic components of $\psi_{1}^{0}$, i.e., from the $\psi_{1}^{0} i$ in the expansion

$$
\psi_{1}^{0}=\psi_{1}^{0 i} Y_{1 i}^{1}+\psi_{1}^{0 i j} Y_{2 i j}^{1}+\ldots
$$


For a different cut and different tetrad, one needs the transformation law to the new $\psi_{1}^{* 0}$ and new $\psi_{1}^{* 0 i}$. Under the tetrad transformation (a null rotation around $n^{a}$ ) to the asymptotically shear-free vector field, $l^{* a}$, Eq. (3.80),

$$
l^{a} \rightarrow l^{* a}=l^{a}-\frac{\bar{L}}{r} m^{a}-\frac{L}{r} \bar{m}^{a}+O\left(r^{-2}\right),
$$

with, from Eqs. (4.30) and (4.31),

$$
\begin{aligned}
u_{\mathrm{B}} & =\xi^{a}(\tau) \hat{l}_{a}(\zeta, \bar{\zeta})+\xi^{i j}(\tau) Y_{2 i j}^{0}(\zeta, \bar{\zeta})+\ldots \\
& =\frac{1}{\sqrt{2}} \xi^{0}(\tau)-\frac{1}{2} \xi^{i}(\tau) Y_{1 i}^{0}(\zeta, \bar{\zeta})+\xi^{i j}(\tau) Y_{2 i j}^{0}(\zeta, \bar{\zeta})+\ldots \\
L\left(u_{\mathrm{B}}, \zeta, \bar{\zeta}\right) & =\xi^{i}(\tau) Y_{1 i}^{1}(\zeta, \bar{\zeta})-6 \xi^{i j}(\tau) Y_{2 i j}^{1}(\zeta, \bar{\zeta})+\ldots
\end{aligned}
$$

the linearized transformation is given by [12]

$$
\psi_{1}^{0 *}=\psi_{1}^{0}-3 L \Psi .
$$

The extraction of the $l=1$ part of $\psi_{1}^{0 *}$ should, in principle, be taken on the new cut given by $u_{\mathrm{B}}=\xi^{a}(\tau) \hat{l}_{a}(\zeta, \bar{\zeta})+\xi^{i j}(\tau) Y_{2 i j}^{0}(\zeta, \bar{\zeta})+\ldots$ with constant $\tau$. However, because of the linearization, the extraction can be taken on the $u_{\mathrm{B}}$ constant cuts. Following the same line of reasoning that led to the definition of center of charge, we demand the vanishing of the $l=1$ part of $\psi_{1}^{0 *}$.

This leads immediately to

$$
\left.\psi_{1}^{0}\right|_{l=1}=\left.3 L \Psi\right|_{l=1},
$$

or, using the decomposition into real and imaginary parts, $\psi_{1}^{0 i}=\psi_{1 R}^{0 i}+i \psi_{1 I}^{0 i}$ and $\xi^{i}\left(u_{\text {ret }}\right)=$ $\xi_{R}^{i}\left(u_{\mathrm{ret}}\right)+i \xi_{I}^{i}\left(u_{\mathrm{ret}}\right)$,

$$
\begin{aligned}
& \psi_{1 R}^{0 i}=-\frac{6 \sqrt{2} G}{c^{2}} M_{\mathrm{Sch}} \xi_{R}^{i}\left(u_{\mathrm{ret}}\right), \\
& \psi_{1 I}^{0 i}=-\frac{6 \sqrt{2} G}{c^{2}} M_{\mathrm{Sch}} \xi_{I}^{i}\left(u_{\mathrm{ret}}\right) .
\end{aligned}
$$

Identifying $[75,53]$ the (intrinsic) angular momentum, either from the conventional linear identification or from the Kerr metric, as

$$
J^{i}=S^{i}=M_{\mathrm{Sch}} c \xi_{I}^{i}
$$

and the mass dipole as

$$
D_{(\text {mass })}^{i}=M_{\mathrm{Sch}} \xi_{R}^{i}
$$

we have

$$
\psi_{1}^{0 i}=-\frac{6 \sqrt{2} G}{c^{2}} D_{(\text {grav })}^{i}=-\frac{6 \sqrt{2} G}{c^{2}}\left(D_{(\text {mass })}^{i}+i c^{-1} J^{i}\right) .
$$

By inserting Eq. (5.19) into Eq. (5.1), taking, respectively, the real and imaginary parts, using Eq. (5.7) and the reality of $\Psi$, we find

$$
P^{i}=M_{\mathrm{Sch}} \xi_{R}^{i \prime} \equiv M_{\mathrm{Sch}} v_{R}^{i},
$$

the kinematic expression of linear momentum and

$$
J^{i \prime}=0,
$$


the conservation of angular momentum.

Finally, from the $l=(0,1)$ parts of Eq. (5.14), we have, at this approximation, that the mass and linear momentum remain constant, i.e., $M=M_{\mathrm{Sch}}=M_{\mathrm{B}}$ and $\delta \Psi^{0}=0$. Thus, we obtain the trivial equations of motion for the center of mass,

$$
M_{\mathrm{Sch}} \xi_{R}^{i \prime \prime}=0 .
$$

The linearization off Schwarzschild, with our identifications, lead to a stationary spinning spacetime object with the standard classical mechanics kinematic and dynamic description. It was the linearization that let to such simplifications, and in Section 6, when nonlinear terms are included (in similar calculations), much more interesting and surprising physical results are found.

\subsection{The Robinson-Trautman metrics}

The algebraically-special type II Robinson-Trautman (RT) metrics are expressed in conventional RT coordinates, $(\tau, r, \zeta, \bar{\zeta}), \tau$ now real, by $[71]$

$$
d s^{2}=2\left(K-\frac{V^{\prime}}{V} r+\frac{\psi_{2}^{0}}{r}\right) d \tau^{2}+2 d \tau d r-r^{2} \frac{2 d \zeta d \bar{\zeta}}{V^{2} P_{0}^{2}},
$$

with

$$
\begin{aligned}
K & =2 V^{2} P_{0}^{2} \partial_{\bar{\zeta}} \partial_{\zeta} \log V P_{0} \\
P_{0} & =1+\zeta \bar{\zeta} \\
\psi_{2}^{0} & =\psi_{2}^{0}(\tau)
\end{aligned}
$$

The unknowns are the Weyl component $\psi_{2}^{0}$ (closely related to the Bondi mass), which is a function only of (real) $\tau$ and the variable, $V(\tau, \zeta, \bar{\zeta}$ ), both of which are variables in the RT equation (see below). There remains the freedom

$$
\tau \rightarrow \tau^{*}=g(\tau),
$$

which often is chosen so that $\psi_{2}^{0}(\tau)=$ constant. However, we make a different choice. In the spherical harmonic expansion of $V$,

$$
V=v^{a} \hat{l}_{a}(\zeta, \bar{\zeta})+v^{i j} Y_{2 i j}^{0}+\ldots,
$$

the $\tau$ is chosen by normalizing the four-vector, $v^{a}$, to one, i.e., $v^{a} v_{a}=1$. The final field equation, the RT equation, is

$$
\psi_{2}^{0 \prime}-3 \psi_{2}^{0} \frac{V^{\prime}}{V^{3}}-V^{3}\left(\check{\partial}_{(\tau)}^{2} \overline{\bar{\partial}}_{(\tau)}^{2} V-V^{-1} \bar{\partial}_{(\tau)}^{2} V \cdot \check{\partial}_{(\tau)}^{2} V\right)=0 .
$$

These spacetimes, via the Goldberg-Sachs theorem, possess a degenerate shear-free PND field, $l^{a}$, that is surface-forming, (i.e., twist free). Using the tetrad constructed from $l^{a}$ we have that the Weyl components are of the form

$$
\begin{aligned}
& \psi_{0}=\psi_{1}=0, \\
& \psi_{2} \neq 0 .
\end{aligned}
$$

Furthermore, the metric contains a 'real timelike world line, $x^{a}=\xi^{a}(\tau)$,' with normalized velocity vector $v^{a}=\xi^{a \prime}$. All of these properties allow us to identify the RT metrics as being analogous to the real Liénard-Wiechert solutions of the Maxwell equations. 
Assuming for the moment that we have integrated the RT equation and know $V=V(\tau, \zeta, \bar{\zeta})$, then, by the integral

$$
u_{\mathrm{B}}=\int V(\tau, \zeta, \bar{\zeta}) d \tau \equiv X_{\mathrm{RT}}(\tau, \zeta, \bar{\zeta}),
$$

the UCF for the RT metrics has been found. The freedom of adding $\alpha(\zeta, \bar{\zeta})$ to the integral is just the supertranslation freedom in the choice of a Bondi coordinate system. From $X_{\mathrm{RT}}(\tau, \zeta, \bar{\zeta})$ a variety of information can be obtained: the Bondi shear, $\sigma^{0}$, is given parametrically by

$$
\begin{aligned}
\sigma^{0}\left(u_{\mathrm{B}}, \zeta, \bar{\zeta}\right) & =\partial_{(\tau)}^{2} X_{\mathrm{RT}}(\tau, \zeta, \bar{\zeta}), \\
u_{\mathrm{B}} & =X_{\mathrm{RT}}(\tau, \zeta, \bar{\zeta}),
\end{aligned}
$$

as well as the angle field $L$ by

$$
\begin{aligned}
L\left(u_{\mathrm{B}}, \zeta, \bar{\zeta}\right) & =\partial_{(\tau)} X_{\mathrm{RT}}(\tau, \zeta, \bar{\zeta}), \\
u_{\mathrm{B}} & =X_{\mathrm{RT}}(\tau, \zeta, \bar{\zeta}) .
\end{aligned}
$$

In turn, from this information the RT metric (in the neighborhood of $\mathfrak{I}^{+}$) can, in principle, be re-expressed in terms of the Bondi coordinate system, though in practice one must revert to approximations. These approximate calculations lead, via the Bondi mass aspect evolution equation, to both Bondi mass loss and to equations of motion for the world line, $x^{a}=\xi^{a}(\tau)$. An alternate approximation for the mass loss and equations of motion is to insert the spherical harmonic expansion of $V$ into the RT equation and look at the lowest harmonic terms. We omit further details aside from mentioning that we come back to these calculations in a more general context in Section 6.

\subsection{Type II twisting metrics}

It was pointed out in the previous section that the RT metrics are the general relativistic analogues of the (real) Liénard-Wiechert Maxwell fields. The type II algebraically-special twisting metrics are the gravitational analogues of the complex Liénard-Wiechert Maxwell fields described earlier. Unfortunately they are far more complicated than the RT metrics. In spite of the large literature and much effort there are very few known solutions and much still to be learned $[41,58,46]$. We give a very brief description of them, emphasizing only the items of relevance to us.

A null tetrad system (and null geodesic coordinates) can be adopted for the type II metrics so that the Weyl tetrad components are

$$
\begin{aligned}
& \psi_{0}=\psi_{1}=0, \\
& \psi_{2} \neq 0 .
\end{aligned}
$$

It follows from the Goldberg-Sachs theorem that the degenerate principal null congruence is geodesic and shear-free. Thus, from the earlier discussions it follows that there is a unique angle field, $L\left(u_{\mathrm{B}}, \zeta, \bar{\zeta}\right)$. As with the complex Liénard-Wiechert Maxwell fields, the type II metrics and Weyl tensors are given in terms of the angle field, $L\left(u_{\mathrm{B}}, \zeta, \bar{\zeta}\right)$. In fact, the entire metric and the field equations (the asymptotic Bianchi identities) can be written in terms of $L$ and a Weyl tensor component (essentially the Bondi mass). Since $L\left(u_{\mathrm{B}}, \zeta, \bar{\zeta}\right)$ describes a unique shear-free NGC, it can be written parametrically in terms of a unique GCF, namely the $\mathrm{UCF} X_{(\text {type II })}(\tau, \zeta, \bar{\zeta})$. So, we have that

$$
\begin{aligned}
L\left(u_{\mathrm{B}}, \zeta, \bar{\zeta}\right) & =\varlimsup_{(\tau)} X_{(\text {type II })}, \\
u_{\mathrm{B}} & =X_{(\text {type II })}(\tau, \zeta, \bar{\zeta}) .
\end{aligned}
$$


Since $X_{\text {(type II) }}(\tau, \zeta, \bar{\zeta})$ can be expanded in spherical harmonics, the $l=(0,1)$ harmonics can be identified with a (unique) complex world line in $\mathcal{H}$-space. The asymptotic Bianchi identities then yield both kinematic equations (for angular momentum and the Bondi linear momentum) and equations of motion for the world line, analogous to those obtained for the Schwarzschild perturbation and the RT metrics. As a kinematic example, the imaginary part of the world line is identified as the intrinsic spin, the same identification as in the Kerr metric,

$$
S^{i}=M_{\mathrm{B}} c \xi_{I}^{i} .
$$

In Section 6, a version of these results will be derived in a far more general context.

Recently, the type II Einstein-Maxwell equations were studied using a slow-motion perturbation expansion around the Reissner-Nördstrom metric, keeping spherical harmonic contributions up to $l=2$. It was found that the above-mentioned world line coincides in this case with that given by the Abraham-Lorentz-Dirac equation, prompting us to consider such spacetimes as 'type II particles' in the same way that one can refer to Reissner-Nördstrom-Schwarzschild or Kerr-Newman 'particles' [52].

\subsection{Asymptotically static and stationary spacetimes}

By defining asymptotically static or stationary spacetimes as those asymptotically-flat spacetimes where the asymptotic variables are 'time' independent, i.e., $u_{\mathrm{B}}$ independent, we can look at our procedure for transforming to the complex center of mass (or complex center of charge). This example, though very special, has the huge advantage in that it can be done exactly, without the use of perturbations [3].

Imposing time independence on the asymptotic Bianchi identities, Eqs. (2.52)-(2.54),

$$
\begin{aligned}
\dot{\psi}_{2}^{0} & =-ð \psi_{3}^{0}+\sigma^{0} \psi_{4}^{0}, \\
\dot{\psi}_{1}^{0} & =-ð \psi_{2}^{0}+2 \sigma^{0} \psi_{3}^{0}, \\
\dot{\psi}_{0}^{0} & =-ð \psi_{1}^{0}+3 \sigma^{0} \psi_{2}^{0},
\end{aligned}
$$

and reality condition

$$
\Psi \equiv \psi_{2}^{0}+\partial^{2} \bar{\sigma}+\sigma \dot{\bar{\sigma}}=\bar{\Psi},
$$

we have, using Eqs. (2.47) and 2.48) with $\dot{\sigma}^{0}=0$, that

$$
\begin{aligned}
\psi_{3}^{0} & =\psi_{4}^{0}=0, \\
\partial \psi_{2}^{0} & =0, \\
\check{\partial} \psi_{1}^{0} & =3 \sigma^{0} \psi_{2}^{0}, \\
\Psi & \equiv \psi_{2}^{0}+\check{\partial}^{2} \bar{\sigma}=\bar{\psi}_{2}^{0}+\bar{ठ}^{2} \sigma=\bar{\Psi} .
\end{aligned}
$$

From Eq. (5.37), we find (after a simple calculation) that the imaginary part of $\psi_{2}^{0}$ is determined by the 'magnetic' [60] part of the Bondi shear (spin-weight $s=2$ ) and thus must contain harmonics only of $l \geq 2$. But from Eq. (5.35), we find that $\psi_{2}^{0}$ contains only the $l=0$ harmonic. From this it follows that the 'magnetic' part of the shear must vanish. The remaining part of the shear, i.e., the 'electric' part, which by assumption is time independent, can be made to vanish by a supertranslation, via the Sachs theorem:

$$
\begin{aligned}
\widehat{u}_{B} & =u_{\mathrm{B}}+\alpha(\zeta, \bar{\zeta}) \\
\widehat{\sigma}(\zeta, \bar{\zeta}) & =\sigma(\zeta, \bar{\zeta})+\partial^{2} \alpha(\zeta, \bar{\zeta})
\end{aligned}
$$


In this Bondi frame, (i.e., frame with a vanishing shear), Eq. (5.36), implies that

$$
\begin{aligned}
\psi_{1}^{0} & =\psi_{1}^{0 i} Y_{1 i}^{1}, \\
\psi_{1}^{0 i} & =-\frac{6 \sqrt{2} G}{c^{2}} D_{\text {(grav) }}^{i}=-\frac{6 \sqrt{2} G}{c^{2}}\left(D_{\text {(mass) }}^{i}+i c^{-1} J^{i}\right),
\end{aligned}
$$

using the conventionally accepted physical identification of the complex gravitational dipole. (Since the shear vanishes, this agrees with probably all the various attempted identifications.)

From the mass identification, $\psi_{2}^{0}$ becomes

$$
\psi_{2}^{0}=-\frac{2 \sqrt{2} G}{c^{2}} M_{\mathrm{B}} .
$$

Since the Bondi shear is zero, the asymptotically shear-free congruences are determined by the same GCFs as in flat spaces, i.e., we have

$$
\begin{aligned}
L\left(u_{\mathrm{B}}, \zeta, \bar{\zeta}\right) & =\partial_{(\tau)} G(\tau, \zeta, \bar{\zeta})=\xi^{a}(\tau) \hat{m}_{a}(\zeta, \bar{\zeta}), \\
u_{\mathrm{B}} & =\xi^{a}(\tau) \hat{l}_{a}(\zeta, \bar{\zeta}) .
\end{aligned}
$$

Our procedure for the identification of the complex center of mass, namely setting $\psi_{1}^{* 0}=0$ in the transformation, Eq. (4.7),

$$
\psi_{1}^{* 0}=\psi_{1}^{0}-3 L \psi_{2}^{0}+3 L^{2} \psi_{3}^{0}-L^{3} \psi_{4}^{0}
$$

leads, after using Eqs. (5.39), (5.34) and (5.42), to

$$
\begin{aligned}
\psi_{1}^{0} & =3 L \psi_{2}^{0}, \\
\psi_{1}^{0 i} & =-\frac{6 \sqrt{2} G}{c^{2}} D_{\text {(grav) }}^{i}, \\
D_{\text {(grav) }}^{i} & =M_{\mathrm{B}} \xi^{i} .
\end{aligned}
$$

From the time independence, $\xi^{i}$, the spatial part of the world line is a constant vector. By a (real) spatial Poincaré transformation (from the BMS group), the real part of $\xi^{i}$ can be made to vanish, while by ordinary rotation the imaginary part of $\xi^{i}$ can be made to point in the threedirection. Using the the gauge freedom in the choice of $\tau$ we set $\xi^{0}(\tau)=\tau$. Then pulling all these items together, we have for the complex world line, the UCF, $L\left(u_{\mathrm{B}}, \zeta, \bar{\zeta}\right)$ and the angular momentum, $J^{i}$ :

$$
\begin{aligned}
\xi^{a}(\tau) & =\left(\tau, 0,0, i \xi^{3}\right) \\
u_{\mathrm{B}} & =X(\tau, \zeta, \bar{\zeta})=\xi^{a}(\tau) \hat{l}_{a}(\zeta, \bar{\zeta}) \equiv \frac{\tau}{\sqrt{2}}-\frac{i}{2} \xi^{3} Y_{1,3}^{0}, \\
L\left(u_{\mathrm{B}}, \zeta, \bar{\zeta}\right) & =i \xi_{I}^{3} Y_{1,3}^{1}, \\
J^{i} & =S^{i}=M_{\mathrm{B}} c \xi^{3} \delta_{3}^{i}=M_{\mathrm{B}} c\left(0,0, \xi^{3}\right)=M_{\mathrm{B}} c \xi_{I}^{i} .
\end{aligned}
$$

Thus, we have the complex center of mass on the complex world line, $z^{a}=\xi^{a}(\tau)$.

These results for the lower multipole moments, i.e., $l=0,1$, are identical to those of the Kerr metric presented earlier! The higher moments are still present (appearing in higher $r^{-1}$ terms in the Weyl tensor) and are not affected by these results. 


\section{Main Results}

We saw in Sections 3 and 4 how shear-free and asymptotically shear-free NGCs determine arbitrary complex analytic world lines in the auxiliary complex $\mathcal{H}$-space (or complex Minkowski space). In the examples from Sections 3 and 5, we saw how, in each of the cases, one could pick out a special GCF, referred to as the UCF, and the associated complex world line by a transformation to the complex center of mass or charge by requiring that the complex dipoles vanish. In the present section we consider the same problem, but now perturbatively for the general situation of asymptotically-flat spacetimes satisfying either the vacuum Einstein or the Einstein-Maxwell equations in the neighborhood of future null infinity. Since the calculations are relatively long and complicated, we give the basic ideas in outline form and then present the final results for Einstein-Maxwell spacetimes without detailed steps.

We begin with the Reissner-Nordström metric, considering both the mass and the charge as zeroth-order quantities, and perturb from it. The perturbation data is considered to be first order and the perturbations themselves are general in the class of analytic asymptotically-flat spacetimes. Though our considerations are for arbitrary mass and charge distributions in the interior, we look at the fields in the neighborhood of $\mathfrak{I}^{+}$. The calculations are carried to second order in the perturbation data. Throughout we use expansions in spherical harmonics and their tensor harmonic versions, but terminate the expansions after $l=2$. Clebsch-Gordon expansions are frequently used; see Appendix C.

\subsection{A brief summary - Before continuing}

Very briefly, for the purpose of organizing the many strands so far developed, we summarize our procedure for finding the complex center of mass. We begin with the gravitational radiation data, the Bondi shear, $\sigma^{0}\left(u_{\mathrm{B}}, \zeta, \bar{\zeta}\right)$ and solve the good-cut equation,

$$
\check{\partial}^{2} Z=\sigma^{0}(Z, \zeta, \bar{\zeta})
$$

with solution $u_{\mathrm{B}}=Z\left(z^{a}, \zeta, \bar{\zeta}\right)$ and the four complex parameters $z^{a}$ defining the solution space. Next we consider an arbitrary complex world line in the solution space, $z^{a}=\xi^{a}(\tau)=\left(\xi^{0}(\tau), \xi^{i}(\tau)\right)$, so that $u_{\mathrm{B}}=Z\left(\xi^{a}(\tau), \zeta, \bar{\zeta}\right)=G(\tau, \zeta, \bar{\zeta})$, a GCF, which can be expanded in spherical harmonics as

$$
\begin{aligned}
u_{\mathrm{B}} & =G(\tau, \zeta, \bar{\zeta})=\xi^{a}(\tau) \hat{l}_{a}(\zeta, \bar{\zeta})+\xi^{i j}(\tau) Y_{2 i j}^{0}+\ldots \\
& =\frac{\xi^{0}(\tau)}{\sqrt{2}}-\frac{1}{2} \xi^{i}(\tau) Y_{1 i}^{0}+\xi^{i j}(\tau) Y_{2 i j}^{0}+\ldots
\end{aligned}
$$

Assuming slow motion and the gauge condition $\xi^{0}(\tau)=\tau$ (see Section 4 ), we have

$$
u_{\mathrm{B}}=\frac{\tau}{\sqrt{2}}-\frac{1}{2} \xi^{i}(\tau) Y_{1 i}^{0}+\xi^{i j}(\tau) Y_{2 i j}^{0}+\ldots
$$

(Though the world line is arbitrary, the quadrupole term, $\xi^{i j}(\tau)$, and higher harmonics, are determined by both the Bondi shear and the world line.)

The inverse function,

$$
\begin{aligned}
\tau & =T\left(u_{\text {ret }}, \zeta, \bar{\zeta}\right), \\
u_{\text {ret }} & =\sqrt{2} u_{\mathrm{B}},
\end{aligned}
$$

can be found by the following iteration process [40]: writing Eq. (6.2) as

$$
\tau=u_{\text {ret }}+F(\tau, \zeta, \bar{\zeta})
$$


with

$$
F(\tau, \zeta, \bar{\zeta})=\frac{\sqrt{2}}{2} \xi^{i}(\tau) Y_{1 i}^{0}(\zeta, \bar{\zeta})-\sqrt{2} \xi^{i j}(\tau) Y_{1 i j}^{0}(\zeta, \bar{\zeta})+\ldots
$$

the iteration relationship, with the zeroth-order iterate, $\tau_{0}=u_{\text {ret }}$, is

$$
\tau_{n}=u_{\mathrm{ret}}+F\left(\tau_{n-1}, \zeta, \bar{\zeta}\right)
$$

To second order, this is

$$
\tau=T\left(u_{\mathrm{ret}}, \zeta, \bar{\zeta}\right)=u_{\mathrm{ret}}+F\left(u_{\mathrm{ret}}+F\left(u_{\mathrm{ret}}, \zeta, \bar{\zeta}\right), \zeta, \bar{\zeta}\right) \approx u_{\mathrm{ret}}+F+F \partial_{u_{\mathrm{ret}}} F,
$$

but for most of our calculations, all that is needed is the first iterate, given by

$$
\tau=T\left(u_{\mathrm{ret}}, \zeta, \bar{\zeta}\right)=u_{\mathrm{ret}}+\frac{\sqrt{2}}{2} \xi^{i}\left(u_{\mathrm{ret}}\right) Y_{1 i}^{0}(\zeta, \bar{\zeta})-\sqrt{2} \xi^{i j}\left(u_{\mathrm{ret}}\right) Y_{1 i j}^{0}(\zeta, \bar{\zeta})
$$

This relationship is, in principle, an important one.

We also have the linearized reality relations - easily found earlier or from Eq. (6.7):

$$
\begin{aligned}
\tau & =s+i \lambda \\
\lambda & =\Lambda(s, \zeta, \bar{\zeta})=\frac{\sqrt{2}}{2} \xi_{I}^{i}(s) Y_{1 i}^{0}-\sqrt{2} \xi_{I}^{i j}(s) Y_{2 i j}^{0}, \\
\tau & =s+i\left(\frac{\sqrt{2}}{2} \xi_{I}^{i}(s) Y_{1 i}^{0}-\sqrt{2} \xi_{I}^{i j}(s) Y_{2 i j}^{0}\right), \\
u_{\text {ret }}^{(R)} & =\sqrt{2} G_{R}(s, \zeta, \bar{\zeta})=\sqrt{2} u_{\mathrm{B}}^{(R)}=s-\frac{\sqrt{2}}{2} \xi_{R}^{i}(s) Y_{1 i}^{0}+\sqrt{2} \xi_{R}^{i j}(s) Y_{2 i j}^{0} .
\end{aligned}
$$

The associated angle field, $L$, and the Bondi shear, $\sigma^{0}$, are given parametrically by

$$
\begin{aligned}
L\left(u_{\mathrm{B}}, \zeta, \bar{\zeta}\right) & =\mathrm{\partial}_{(\tau)} G(\tau, \zeta, \bar{\zeta}) \\
& =\xi^{i}(\tau) Y_{1 i}^{1}-6 \xi^{i j}(\tau) Y_{2 i j}^{1}+\ldots
\end{aligned}
$$

and

$$
\begin{aligned}
\sigma^{0}\left(u_{\mathrm{B}}, \zeta, \bar{\zeta}\right) & =\check{\partial}_{(\tau)}^{2} G(\tau, \zeta, \bar{\zeta}), \\
& =24 \xi^{i j}(\tau) Y_{2 i j}^{2}+\ldots,
\end{aligned}
$$

using the inverse to $u_{\mathrm{B}}=G(\tau, \zeta, \bar{\zeta})$, Eq. (6.7). The asymptotically shear-free NGC is given by performing the null rotation

$$
\begin{aligned}
l^{* a} & =l^{a}+b \bar{m}^{a}+\bar{b} m^{a}+b \bar{b} n^{a}, \\
m^{* a} & =m^{a}+b n^{a}, \\
n^{* a} & =n^{a}, \\
b & =-L / r+O\left(r^{-2}\right) .
\end{aligned}
$$

As stated in Eqs. (4.6) - (4.10), under (6.14) the transformed asymptotic Weyl tensor becomes

$$
\begin{aligned}
& \psi_{0}^{* 0}=\psi_{0}^{0}-4 L \psi_{1}^{0}+6 L^{2} \psi_{2}^{0}-4 L^{3} \psi_{3}^{0}+L^{4} \psi_{4}^{0}, \\
& \psi_{1}^{* 0}=\psi_{1}^{0}-3 L \psi_{2}^{0}+3 L^{2} \psi_{3}^{0}-L^{3} \psi_{4}^{0}, \\
& \psi_{2}^{* 0}=\psi_{2}^{0}-2 L \psi_{3}^{0}+L^{2} \psi_{4}^{0}, \\
& \psi_{3}^{* 0}=\psi_{3}^{0}-L \psi_{4}^{0} \\
& \psi_{4}^{* 0}=\psi_{4}^{0} .
\end{aligned}
$$


The procedure for finding the complex center of mass is centered on Eq. (6.16), where we search for and set to zero the $l=1$ harmonic in $\psi_{1}^{* 0}$ on a $\tau=$ constant slice. This determines the complex center-of-mass world line and singles out a particular GCF referred to as the UCF,

$$
u_{\mathrm{B}}=X(\tau, \zeta, \bar{\zeta})=G(\tau, \zeta, \bar{\zeta})
$$

with the real version,

$$
u_{\mathrm{ret}}^{(R)}=X_{R}(s, \zeta, \bar{\zeta})=G_{R}(s, \zeta, \bar{\zeta}),
$$

for the gravitational field in the general asymptotically-flat case.

For the case of the Einstein-Maxwell fields, in general there will be two complex world lines and two associated UCFs, one for the center of charge, the other for the center of mass. For later use we note that the gravitational world line will be denoted by $\xi^{a}$, while the electromagnetic world line by $\eta^{a}$. Later we consider the special case when the two world lines and the two UCFs coincide, i.e., $\xi^{a}=\eta^{a}$.

From the assumption that $\sigma^{0}$ and $L$ are first order and, from Eqs. (2.48) and (2.49) (e.g., $\left.\psi_{3}^{0}=\partial \dot{\bar{\sigma}}^{0}\right)$, Eq. (6.16), to second order, is

$$
\psi_{1}^{* 0}=\psi_{1}^{0}-3 L\left(\Psi-\check{\partial}^{2} \bar{\sigma}^{0}\right),
$$

where $\psi_{2}^{0}$ has been replaced by the mass aspect (2.50): $\Psi \approx \psi_{2}^{0}+\check{\partial}^{2} \bar{\sigma}^{0}$.

Using the spherical harmonic expansions (see Eqs. (6.12) and (6.13)),

$$
\begin{aligned}
\Psi & =\Psi^{0}+\Psi^{i} Y_{1 i}^{0}+\Psi^{i j} Y_{2 i j}^{0}+\ldots, \\
\psi_{1}^{0} & =\psi_{1}^{0 i} Y_{1 i}^{1}+\psi_{1}^{0 i j} Y_{2 i j}^{1}+\ldots, \\
\psi_{1}^{* 0} & =\psi_{1}^{* 0 i} Y_{1 i}^{1}+\psi_{1}^{* 0 i j} Y_{2 i j}^{1}+\ldots, \\
L\left(u_{\mathrm{B}}, \zeta, \bar{\zeta}\right) & =\xi^{i}(\tau) Y_{1 i}^{1}-6 \xi^{i j}(\tau) Y_{2 i j}^{1}+\ldots, \\
\sigma^{0}\left(u_{\mathrm{B}}, \zeta, \bar{\zeta}\right) & =24 \xi^{i j}(\tau) Y_{2 i j}^{2}+\ldots
\end{aligned}
$$

and remembering that $\Psi^{0}$ is zeroth order, Eq. (6.22), becomes

$$
\psi_{1}^{* 0}=\psi_{1}^{0}-3\left[\xi^{i}(\tau) Y_{1 i}^{1}-6 \xi^{i j}(\tau) Y_{2 i j}^{1}\right]\left[\Psi^{0}+\Psi^{i} Y_{1 i}^{0}+\left\{\Psi^{i j}-24 \bar{\xi}^{i j}(\tau)\right\} Y_{2 i j}^{0}\right]
$$

or, re-arranging and performing the relevant Clebsch-Gordon expansions,

$$
\begin{aligned}
\psi_{1}^{0}= & \psi_{1}^{* 0}+3 \Psi^{0} \xi^{i} Y_{1 i}^{1}+\frac{3 \sqrt{2} i}{2} \xi^{k} \Psi^{j} \epsilon_{k j i} Y_{1 i}^{1}-\frac{108}{5} \xi^{i k} \Psi^{k} Y_{1 i}^{1}-\frac{18}{5} \xi^{k}\left(\Psi^{i k}-24 \bar{\xi}^{i k}\right) Y_{1 i}^{1} \\
& -\frac{216 \sqrt{2} i}{5} \xi^{k j}\left(\Psi^{k l}-24 \bar{\xi}^{k l}\right) \epsilon_{j l i} Y_{1 i}^{1}+l \geq 2 \text { harmonic contributions } .
\end{aligned}
$$

Note that though Eq. (6.28) depends initially on both $\tau$ and $u_{\text {ret }}$, with $\tau=T\left(u_{\text {ret }}, \zeta, \bar{\zeta}\right)$, we will eventually replace all the $u_{\mathrm{B}}$ (or $u_{\text {ret }}$ ) by their expressions in terms of $\tau$, using Eq. (6.2). The transformation equation is then a function only of $\tau$ and $(\zeta, \bar{\zeta})$, at least to the given order in our perturbative framework.

This equation, though complicated and unattractive, is our main source of information concerning the complex center-of-mass world line. The information is extracted in the following way: Considering only the $l=1$ harmonics at constant $\tau$ in Eq. (6.28), we set the $l=1$ harmonics of $\psi_{1}^{* 0}$ (with constant $\tau$ ) to zero (i.e., $\psi_{1}^{* 0 i}=0$ ). The three resulting relations are used to determine the three spatial components, $\xi^{k}(\tau)$, of $\xi^{a}(\tau)$ (with $\xi^{0}=\tau$ ). This fixes the complex center of mass in terms of $\psi_{1}^{0 i}, \Psi^{0}, \Psi^{i}$, and other data which is readily interpreted physically. Alternatively it allow us to express $\psi_{1}^{0 i}$ in terms of the $\xi^{a}(\tau)$.

Extracting this information takes a bit of effort. 


\subsection{The complex center-of-mass world line}

Before trying to determine the $l=1$ harmonics of Eq. (6.28), several comments and repetitions (for emphasis) are in order:

1. As previously noted, Eq. (6.28) is a function of both $\tau$ (via the $\xi^{i}, \xi^{i j}$ ) and $u_{\text {ret }}$ (via the $\psi_{1}^{0 i}$ and $\Psi$ ). The extraction of the $l=1$ part of $\psi_{1}^{* 0}$ must be taken on the constant $\tau$ cuts. In other words $u_{\text {ret }}$ must be eliminated by using Eq. (6.2).

2. This elimination of $u_{\mathrm{B}}$ (or $u_{\mathrm{ret}}$ ) is done in the linear terms via the expansion:

$$
\begin{aligned}
\eta\left(u_{\mathrm{ret}}\right) & =\eta\left(\tau-\frac{\sqrt{2}}{2} \xi_{R}^{i}(\tau) Y_{1 i}^{0}+\sqrt{2} \xi_{R}^{i j}(\tau) Y_{2 i j}^{0}\right) \\
& \approx \eta(\tau)-\frac{\sqrt{2}}{2} \eta(s)^{\prime}\left[\xi_{R}^{i}(s) Y_{1 i}^{0}-2 \xi_{R}^{i j}(s) Y_{2 i j}^{0}\right] \\
u_{\text {ret }} & =\tau-\frac{\sqrt{2}}{2} \xi^{i}(\tau) Y_{1 i}^{0}+\sqrt{2} \xi^{i j}(\tau) Y_{2 i j}^{0}+\ldots
\end{aligned}
$$

In the nonlinear terms we can simply use

$$
u_{\mathrm{ret}}=\tau .
$$

3. In the Clebsch-Gordon expansions of the harmonic products, though we need both the $l=1$ and $l=2$ terms in the calculation, we keep at the end only the $l=1$ terms for the $\psi_{1}^{0 i}$. (Note that there are no $l=0$ terms since $\psi_{1}^{* 0}$ is spin weight $s=1$.)

4. For completeness, we have included into the calculations Maxwell fields with both a complex dipole (electric and magnetic), $D_{\mathbb{C}}^{i}=q \eta^{i}=q\left(\eta_{R}^{i}+i \eta_{I}^{i}\right)$ and complex quadrupole (electric and magnetic) fields $Q_{\mathbb{C}}^{k j}=Q_{E}^{k j}+i Q_{M}^{k j}$.

We begin by focusing on the $l=1$ portion of the right-hand-side of Eq. (6.28) in the complex center of mass frame. Assuming that $\psi_{1}^{* 0 i}=0$, we see that all remaining terms on this side of the equation are nonlinear, so we can simply make the replacement $u_{\mathrm{ret}} \rightarrow \tau$. On the left-hand side of the equation, extracting the $l=1$ component of $\psi_{1}^{0}$ on a constant $\tau$ slice is more complicated though; using Eq. (6.30), we have that

$$
\psi_{1}^{0}\left(u_{\mathrm{ret}}\right)=\psi_{1}^{0}(\tau)-\frac{\sqrt{2}}{2} \psi_{1}^{0}(\tau)^{\prime} \xi^{i}(\tau) Y_{1 i}^{1}+\sqrt{2} \psi_{1}^{0}(\tau)^{\prime} \xi^{i j}(\tau) Y_{2 i j}^{1} .
$$

Using the Bianchi identity (2.53) and inserting the proper factor of $\sqrt{2}$ to account for the retarded Bondi time, we have that (to second order)

$$
\psi_{1}^{0 \prime}=-\frac{\sqrt{2}}{2} ð \Psi+\frac{\sqrt{2}}{2} \partial^{3} \bar{\sigma}^{0}+\sqrt{2} k \phi_{1}^{0} \bar{\phi}_{2}^{0} .
$$

As $\psi_{1}^{0 \prime}$ enters Eq. (6.31) only in nonlinear terms, we only need to extract the linear portion of this Bianchi identity. Recalling (suppressing factors of $c$ for the time being) that

$$
\phi_{1}^{0}=q+\sqrt{2} q \eta^{i \prime} Y_{1 i}^{0}+\frac{\sqrt{2}}{6} Q_{\mathbb{C}}^{i j \prime \prime} Y_{2 i j}^{0}, \quad \phi_{2}^{0}=-2 q \eta^{i \prime \prime} Y_{1 i}^{1}-\frac{1}{3} Q_{\mathbb{C}}^{i j \prime \prime \prime} Y_{2 i j}^{1},
$$

we readily determine that

$$
\psi_{1}^{0 \prime}=\sqrt{2} \Psi^{i} Y_{1 i}^{1}+3 \sqrt{2}\left(\Psi^{i j}-24 \bar{\xi}^{i j}\right) Y_{2 i j}^{1}-2 \sqrt{2} k q^{2} \bar{\eta}^{i \prime \prime} Y_{1 i}^{1}-\frac{\sqrt{2} k q}{3} \bar{Q}_{\mathbb{C}}^{i j \prime \prime \prime} Y_{2 i j}^{1} .
$$


Feeding (6.32) into Eq. (6.31) and performing the relevant Clebsch-Gordon expansions, we find:

$$
\begin{aligned}
\left.\psi_{1}^{0}\left(u_{\mathrm{ret}}\right)\right|_{l=1}= & \psi_{1}^{0 i}-\frac{\sqrt{2} i}{2} \Psi^{j} \xi^{k} \epsilon_{j k i}+\frac{864}{5} \xi^{j} \bar{\xi}^{j i}-\frac{12}{5} \Psi^{j} \xi^{j i}+\frac{3456 \sqrt{2} i}{5} \xi^{l j} \bar{\xi}^{l k} \epsilon_{j k i} \\
& +\sqrt{2} i k q^{2} \xi^{k} \bar{\eta}^{j \prime \prime} \epsilon_{j k i}+\frac{24}{5} k q^{2} \xi^{j i} \bar{\eta}^{j \prime \prime}+\frac{2}{5} k q \xi^{j} \bar{Q}_{\mathbb{C}}^{j i \prime \prime \prime}+\frac{24 \sqrt{2} i}{5} k q \xi^{l j} \bar{Q}_{\mathbb{C}}^{l k \prime \prime \prime} \epsilon_{j k i} .(6.3
\end{aligned}
$$

Here, we have implicitly used the fact that, to our level of approximation, $\Psi^{i j}=-24 \bar{\xi}^{i j}$.

We can now incorporate this into Eq. (6.28) to obtain the full complex center of mass equation as a function of $\tau$. The $l=0,1$ components of the mass aspect are replaced by the expressions

$$
\Psi^{0}=-\frac{2 \sqrt{2} G}{c^{2}} M_{\mathrm{B}}, \quad \Psi^{i}=-\frac{6 G}{c^{3}} P^{i}, \quad \Psi^{i j}=-24 \bar{\xi}^{i j} ;
$$

we insert $k=2 G c^{-4}$ and the appropriate factors of $c$ elsewhere, at which point Eq. (6.28) can be re-expressed in a manner that determines the complex center of mass, with all terms being functions of $\tau$ :

$$
\begin{aligned}
\psi_{1}^{0 i}= & -\frac{6 \sqrt{2} G}{c^{2}} M_{\mathrm{B}} \xi^{i}+\frac{6 \sqrt{2} i}{c^{3}} G P^{k} \xi^{j} \epsilon_{k j i}-\frac{576 G}{5 c^{3}} P^{k} \xi^{k i}+\frac{6912 \sqrt{2} i}{5} \xi^{l j} \bar{\xi}^{l k} \epsilon_{j k i} \\
& -\frac{2 \sqrt{2} i}{c^{6}} G q^{2} \xi^{k} \bar{\eta}^{j \prime \prime} \epsilon_{j k i}-\frac{48 G}{5 c^{6}} q^{2} \xi^{j i} \bar{\eta}^{j \prime \prime}-\frac{4 G}{5 c^{7}} q \xi^{j} \bar{Q}_{\mathbb{C}}^{j i \prime \prime \prime}-\frac{16 \sqrt{2} i}{5 c^{7}} G q \xi^{l j} \bar{Q}_{\mathbb{C}}^{l k \prime \prime \prime} \epsilon_{j k i} .
\end{aligned}
$$

Note that the linear term

$$
\psi_{1}^{0 i}=-\frac{6 \sqrt{2} G}{c^{2}} M_{B} \xi^{i}=3 \Psi^{0} \xi^{i}
$$

coincides with the earlier results in the stationary case, Eq. (5.44).

Now, we recall our identification for the complex gravitational dipole,

$$
\psi_{1}^{0 i}(\tau)=-\frac{6 \sqrt{2} G}{c^{2}}\left(D_{\text {(mass) }}^{i}+i c^{-1} J^{i}\right),
$$

as well as the identification between the $l=2$ harmonic coefficient of the UCF and the gravitational quadrupole:

$$
\xi^{i j}=\frac{\sqrt{2} G}{24 c^{4}} Q_{\mathrm{Grav}}^{i j \prime \prime}=\frac{\sqrt{2} G}{24 c^{4}}\left(Q_{\mathrm{Mass}}^{i j \prime \prime}+i Q_{\mathrm{Spin}}^{i j \prime \prime}\right) .
$$

Feeding these into (6.34), we can separate out the real and imaginary parts via (6.35) to obtain expressions for the mass dipole and angular momentum, our primary results:

$$
\begin{aligned}
& D_{\text {(mass) }}^{i}=M_{\mathrm{B}} \xi_{R}^{i}-c^{-1} P^{k} \xi_{I}^{j} \epsilon_{j k i}+\frac{4 G}{5 c^{5}} P^{k} Q_{\text {Mass }}^{k i \prime \prime}+\frac{2 G}{5 c^{6}} Q_{\text {Spin }}^{l j \prime \prime} Q_{\text {Mass }}^{l k \prime \prime} \epsilon_{j k i} \\
& +\frac{q^{2}}{3 c^{4}}\left(\xi_{R}^{k} \eta_{I}^{j \prime \prime}-\xi_{I}^{k} \eta_{R}^{j \prime \prime}\right) \epsilon_{j k i}+\frac{G q^{2}}{15 c^{8}}\left(\eta_{R}^{j \prime \prime} Q_{\text {Mass }}^{j i \prime \prime}+\eta_{I}^{j \prime \prime} Q_{\text {Spin }}^{j i \prime \prime}\right) \\
& +\frac{\sqrt{2} q}{15 c^{5}}\left(\xi_{R}^{j} Q_{E}^{j i \prime \prime \prime}+\xi_{I}^{j} Q_{M}^{j i \prime \prime \prime}\right) \\
& +\frac{\sqrt{2} G q}{45 c^{9}}\left(Q_{\text {Mass }}^{l j \prime \prime} Q_{M}^{l k \prime \prime \prime}-Q_{\text {Spin }}^{l j \prime \prime} Q_{E}^{l k \prime \prime \prime}\right) \epsilon_{j k i} \\
& J^{i}=c M_{\mathrm{B}} \xi_{I}^{i}+\xi_{R}^{j} P^{k} \epsilon_{j k i}+\frac{4 G}{5 c^{4}} P^{k} Q_{\mathrm{Spin}}^{k i \prime \prime}+\frac{q^{2}}{3 c^{3}}\left(\xi_{R}^{k} \eta_{R}^{j \prime \prime}+\xi_{I}^{k} \eta_{I}^{j \prime \prime}\right) \epsilon_{j k i}+\frac{\sqrt{2} q}{15 c^{4}}\left(\xi_{I}^{j} Q_{E}^{j i \prime \prime \prime}-\xi_{R}^{j} Q_{M}^{j i \prime \prime \prime}\right) \\
& +\frac{G q^{2}}{15 c^{7}}\left(\eta_{R}^{j \prime \prime} Q_{\text {Spin }}^{j i \prime \prime}-\eta_{I}^{j \prime \prime} Q_{\text {Mass }}^{j i \prime \prime}\right)+\frac{\sqrt{2} G q}{45 c^{8}}\left(Q_{\text {Mass }}^{l j \prime \prime} Q_{E}^{l k \prime \prime \prime}+Q_{\text {Spin }}^{l j \prime \prime} Q_{M}^{l k \prime \prime \prime}\right) \epsilon_{j k i} .
\end{aligned}
$$


Though these results are discussed at greater length later, we point out that Eqs. (6.37) and (6.38) already contains terms of obvious physical interest. Note that the first two items in $J^{i}$ are the spin,

$$
\vec{S}=c M_{\mathrm{B}} \vec{\xi}_{I}
$$

(identified via the special case of the Kerr-Newman metric) and the orbital angular momentum

$$
\vec{L}=\vec{\xi}_{R} \times \vec{P} .
$$

The mass dipole $D_{\text {(mass) }}^{i}$ has the conventional term $M_{\mathrm{B}} \vec{\xi}_{R}$ and a momentum-spin coupling term (which appears to be new):

$$
D_{\text {(mass) }}=M_{\mathrm{B}} \vec{\xi}_{R}+\frac{1}{c^{2} M_{\mathrm{B}}} \vec{P} \times \vec{S}+\cdots
$$

We will see shortly that there is also a great deal of physical content to be found in the nonlinear terms of Eq. (6.34).

\subsection{The evolution of the complex center of mass}

The evolution of the mass dipole and the angular momentum, defined from the $\psi_{1}^{0 i}$, Eq. (6.34) and Eqs. (6.37) with (6.38), is determined via the Bianchi identity

$$
\dot{\psi}_{1}^{0}=-ð \psi_{2}^{0}+2 \sigma^{0} \psi_{3}^{0}+2 k \phi_{1}^{0} \bar{\phi}_{2}^{0} .
$$

This relationship allows us the determine - kinematically - the Bondi momentum in terms of the dipole and the complex world line.

By extracting the $l=1$ harmonic from Eq. (6.42), a process which involves several ClebschGordon expansions, we find

$$
\begin{aligned}
\psi_{1}^{0 i \prime}=\sqrt{2} c \Psi^{i} & +\frac{1728 \sqrt{2} i}{5} \xi^{k j} \bar{\xi}^{k l \prime}-\frac{4 \sqrt{2} G}{c^{5}} q^{2} \bar{\eta}^{i \prime \prime}-\frac{4 \sqrt{2} i}{c^{6}} G q^{2} \eta^{j \prime} \bar{\eta}^{k \prime \prime} \epsilon_{k j i} \\
& +\frac{8 G}{5 c^{7}} q \bar{\eta}^{j \prime \prime} Q_{\mathbb{C}}^{j i \prime \prime}-\frac{8 G}{5 c^{7}} q \eta^{j \prime} \bar{Q}_{\mathbb{C}}^{j i \prime \prime \prime}-\frac{8 \sqrt{2} i}{15 c^{8}} G Q_{\mathbb{C}}^{k j \prime \prime} \bar{Q}_{\mathbb{C}}^{k l \prime \prime \prime} \epsilon_{l j i} .
\end{aligned}
$$

Using our various identifications for the complex gravitational dipole, the Bondi momentum, and the complex gravitational quadrupole, Eq. (6.43) can be written as

$$
\begin{aligned}
\left(D_{\text {(mass) }}^{i}+i c^{-1} J^{i}\right)^{\prime}= & P^{i}-\frac{12 i}{5 c^{6}} G Q_{\mathrm{Grav}}^{k j \prime \prime} \bar{Q}_{\mathrm{Grav}}^{k l \prime \prime} \epsilon_{j l i}+\frac{2 q^{2}}{3 c^{3}} \bar{\eta}^{i \prime \prime}+\frac{2 i}{3 c^{4}} q^{2} \eta^{j \prime} \bar{\eta}^{k \prime \prime} \epsilon_{k j i} \\
& -\frac{2 \sqrt{2}}{15 c^{5}} q^{j} \bar{\eta}^{\prime \prime} Q_{\mathbb{C}}^{j i \prime \prime}+\frac{2 \sqrt{2}}{15 c^{5}} q \eta^{j \prime} \bar{Q}_{\mathbb{C}}^{j i \prime \prime \prime}+\frac{4 i}{45 c^{6}} Q_{\mathbb{C}}^{k j \prime \prime} \bar{Q}_{\mathbb{C}}^{k l \prime \prime \prime} \epsilon_{l j i},
\end{aligned}
$$

or in terms of real and imaginary parts:

$$
\begin{aligned}
D_{\text {(mass) }}^{i \prime} & =P^{i}+\frac{2 q^{2}}{3 c^{3}} \eta_{R}^{i \prime \prime}+\frac{2 q^{2}}{3 c^{4}}\left(\eta_{R}^{j \prime} \eta_{I}^{k \prime}\right)^{\prime} \epsilon_{k j i}-\frac{12 G}{5 c^{6}}\left(Q_{\text {Mass }}^{k j \prime \prime} Q_{\text {Spin }}^{k l \prime \prime}\right)^{\prime} \epsilon_{j l i} \\
& +\frac{2 \sqrt{2} q}{15 c^{5}}\left(\eta_{R}^{j \prime} Q_{E}^{j i \prime \prime \prime}-\eta_{R}^{j \prime \prime} Q_{E}^{j i \prime \prime}+\eta_{I}^{j \prime} Q_{M}^{j i \prime \prime \prime}-\eta_{I}^{j \prime \prime} Q_{M}^{j i \prime \prime}\right)+\frac{4}{45 c^{6}}\left(Q_{E}^{k j \prime \prime} Q_{M}^{k l \prime \prime}\right)^{\prime} \epsilon_{l j i},(6 . \\
J^{i \prime}= & -\frac{2 q^{2}}{3 c^{2}} \eta_{I}^{i \prime \prime}+\frac{2 q^{2}}{3 c^{3}}\left(\eta_{R}^{j \prime} \eta_{R}^{k \prime \prime}+\eta_{I}^{j \prime} \eta_{I}^{k \prime \prime}\right) \epsilon_{k j i}-\frac{12 G}{5 c^{5}}\left(Q_{\text {Mass }}^{k j \prime \prime} Q_{\text {Mass }}^{k l \prime \prime \prime}+Q_{\text {Spin }}^{k j \prime \prime} Q_{\text {Spin }}^{k l \prime \prime}\right) \epsilon_{j l i} \\
& +\frac{2 \sqrt{2} q}{15 c^{4}}\left(\eta_{I}^{j \prime} Q_{E}^{j i \prime \prime}-\eta_{R}^{j \prime} Q_{M}^{j i \prime \prime}\right)^{\prime}+\frac{4}{45 c^{5}}\left(Q_{E}^{k j \prime \prime} Q_{E}^{k l \prime \prime \prime}+Q_{M}^{k j \prime \prime} Q_{M}^{k l \prime \prime \prime}\right) \epsilon_{l j i} .
\end{aligned}
$$


Eq. (6.46), which is the conservation of angular momentum, has several things to note. As there are two terms appearing as total derivatives (the first and fourth), it might be more natural to include them in an alternative definition of angular momentum [8]:

$$
J_{T}^{i}=J^{i}+\frac{2 q^{2}}{3 c^{2}} \eta_{I}^{i \prime}-\frac{2 \sqrt{2} q}{15 c^{4}}\left(\eta_{I}^{j \prime} Q_{E}^{j i \prime \prime}-\eta_{R}^{j \prime} Q_{M}^{j i \prime \prime}\right) .
$$

This results in an alternative flux law for angular momentum conservation,

$$
\begin{aligned}
J_{T}^{i \prime}= & (\mathrm{Flux})_{T}^{i} \\
(\mathrm{Flux})_{T}^{i}= & \frac{2 q^{2}}{3 c^{3}}\left(\eta_{R}^{j \prime} \eta_{R}^{k \prime \prime}+\eta_{I}^{j \prime} \eta_{I}^{k \prime \prime}\right) \epsilon_{k j i}-\frac{12 G}{5 c^{5}}\left(Q_{\text {Mass }}^{k j \prime \prime} Q_{\text {Mass }}^{k l \prime \prime \prime}+Q_{\text {Spin }}^{k j \prime \prime} Q_{\text {Spin }}^{k l \prime \prime \prime}\right) \epsilon_{j l i} \\
& +\frac{4}{45 c^{5}}\left(Q_{E}^{k j \prime \prime} Q_{E}^{k l \prime \prime \prime}+Q_{M}^{k j \prime \prime} Q_{M}^{k l \prime \prime \prime}\right) \epsilon_{l j i},
\end{aligned}
$$

whose terms appear to agree with the known angular momentum flux due to gravitational quadrupole and electromagnetic dipole and quadrupole radiation [43].

As for the evolution equation for the mass dipole (6.45), we can obtain an expression for the Bondi linear momentum by taking the derivative (with respect to retarded Bondi time) of Eq. (6.37) to eliminate $D_{(\text {mass })}^{i}$ and find:

$$
P^{i}=M_{\mathrm{B}} \xi_{R}^{i \prime}-\frac{2 q^{2}}{3 c^{3}} \eta_{R}^{i \prime \prime}+\mathfrak{P}_{1}^{i}+\mathfrak{P}_{2}^{i}+\mathfrak{P}_{3}^{i}
$$

where $\mathfrak{P}_{1}^{i}, \mathfrak{P}_{2}^{i}$ and $\mathfrak{P}_{3}^{i}$ are nonlinear terms representing dipole-dipole, dipole-quadrupole and quadrupole-quadrupole coupling respectively,

$$
\begin{aligned}
\mathfrak{P}_{1}^{i}= & \frac{q^{2}}{3 c^{4}}\left(3 \eta_{R}^{k \prime \prime} \xi_{I}^{j}-\eta_{I}^{j \prime \prime} \xi_{r}^{k}-2 \eta_{R}^{k \prime} \eta_{I}^{j \prime}\right)^{\prime} \epsilon_{j k i}-\frac{M_{\mathrm{B}}}{c}\left(\xi_{R}^{k \prime \prime} \xi_{I}^{j}\right)^{\prime} \epsilon_{j k i}, \\
\mathfrak{P}_{2}^{i}= & \frac{4 G}{5 c^{5}} M_{\mathrm{B}}\left(\xi_{R}^{k \prime} Q_{\mathrm{Mass}}^{k i \prime \prime}\right)^{\prime}+\frac{2 \sqrt{2} q}{15 c^{5}}\left(\eta_{R}^{j \prime \prime} Q^{j i \prime \prime}-\eta_{R}^{j \prime} Q_{E}^{j i \prime \prime \prime}+\eta_{I}^{j \prime \prime} Q_{M}^{j i \prime \prime}-\eta_{I}^{j \prime} Q_{M}^{j i \prime \prime \prime}\right) \\
& +\frac{\sqrt{2} q}{15 c^{5}}\left(\xi_{R}^{j} Q_{E}^{j i \prime \prime \prime}+\xi_{I}^{j} Q_{M}^{j i \prime \prime \prime}\right)^{\prime}+\frac{G q^{2}}{15 c^{8}}\left(\eta_{I}^{j \prime \prime} Q_{\mathrm{Spin}}^{j i \prime \prime}-7 \eta_{R}^{j \prime \prime} Q_{\text {Mass }}^{j i \prime \prime}\right)^{\prime}, \\
\mathfrak{P}_{3}^{i}= & \frac{2 G}{c^{6}}\left(Q_{\text {Mass }}^{k j \prime \prime} Q_{\mathrm{Spin}}^{k l \prime \prime}\right)^{\prime} \epsilon_{j l i}+\frac{4}{45 c^{6}}\left(Q_{E}^{k j \prime \prime} Q_{M}^{k l \prime \prime}\right)^{\prime} \epsilon_{j l i}+\frac{\sqrt{2} G q}{45 c^{9}}\left(Q_{\mathrm{Mass}}^{l j \prime \prime} Q_{M}^{l k \prime \prime \prime}-Q_{\mathrm{Spin}}^{l j \prime \prime} Q_{E}^{l k \prime \prime \prime}\right)^{\prime} \epsilon_{j k i} .
\end{aligned}
$$

Remark 11. In the calculation leading to Eq. (6.49), nonlinear terms with $P^{i}$ (or its derivatives) were replaced by the linear expression $P^{i} \approx M_{\mathrm{B}} \xi_{R}^{i \prime}-\frac{2}{3} c^{-3} q^{2} \eta_{R}^{i \prime \prime}$. Additionally, we have neglected the time derivatives of the Bondi mass, $M_{\mathrm{B}}^{\prime}$; this is because, as we shall see momentarily, these derivatives are themselves second order quantities and hence give a vanishing contribution to Eq. (6.49) at our level of approximation.

\section{Physical Content}

- The first term of $P^{i}$ is the standard Newtonian kinematic expression for the linear momentum, $M \vec{v}$.

- The second term, $-\frac{2}{3} c^{-3} q^{2} \eta_{R}^{i \prime \prime}$, which is a contribution from the second derivative of the electric dipole moment, $q \eta_{R}^{i}$, plays a special role for the case when the complex center of mass coincides with the complex center of charge, $\eta^{a}=\xi^{a}$. In this case, the second term is exactly the contribution to the momentum that yields the classical radiation reaction force of classical electrodynamics [43]. 
- Many of the remaining terms in $P^{i}$, though apparently second order, are really of higher order when the dynamics are considered. Others involve quadrupole interactions, which contain high powers of $c^{-1}$.

- In the expression for $J^{i}$ we have already identified, in earlier discussions, the first two terms $M_{\mathrm{B}} c \xi_{I}^{j}$ and $M_{\mathrm{B}} \xi_{R}^{k \prime} \xi_{R}^{i} \epsilon_{i k j}$ as the intrinsic spin angular momentum and the orbital angular momentum. The further terms, a spin-spin, spin-quadrupole and quadrupole-quadrupole interaction terms, are considerably smaller.

- As mentioned earlier, in Eq. (6.46) we see that there are five flux terms, the second is from the gravitational quadrupole flux, the third and fifth are from the classical electromagnetic dipole and electromagnetic quadrupole flux, while the fourth come from dipole-quadrupole coupling. The Maxwell dipole part is identical to that derived from pure Maxwell theory [43]. We emphasize that this angular momentum flux law has little to do directly with the chosen definition of angular momentum. The imaginary part of the Bianchi identity (6.42), with the reality condition $\Psi=\bar{\Psi}$, is the angular momentum conservation law. How to identify the different terms, i.e., identifying the time derivative of the angular momentum and the flux terms, comes from different arguments. The identification of the Maxwell contribution to total angular momentum and the flux contain certain arbitrary assignments: some terms on the left-hand side of the equation, i.e., terms with a time derivative, could have been moved onto the right-hand side and been called 'flux' terms. However, our assignments were governed by the question of what terms appeared most naturally to be on different sides. The first term appears to be a new prediction.

- The angular momentum conservation law can be considered as the evolution equation for the imaginary part of the complex world line, i.e., $\xi_{I}^{i}\left(u_{\text {ret }}\right)$. The evolution for the real part is found from the Bondi energy-momentum loss equation.

- In the special case where the complex centers of mass and charge coincide, $\eta^{a}=\xi^{a}$, we have a rather attractive identification: since now the magnetic dipole moment is given by $D_{M}^{i}=q \xi_{I}^{i}$ and the spin by $S^{i}=M_{\mathrm{B}} c \xi_{I}^{i}$, we have that the gyromagnetic ratio is

$$
\frac{\left|S^{i}\right|}{\left|D_{M}^{i}\right|}=\frac{M_{\mathrm{B}} c}{q}
$$

leading to the Dirac value of $g$, i.e., $g=2$.

\subsection{The evolution of the Bondi energy-momentum}

Finally, to obtain the equations of motion, we substitute the kinematic expression for $P^{i}$ into the Bondi evolution equation, the Bianchi identity, Eq. (2.52);

$$
\dot{\psi}_{2}^{0}=-ð \psi_{3}^{0}+\sigma^{0} \psi_{4}^{0}+k \phi_{2}^{0} \bar{\phi}_{2}^{0},
$$

or its much more useful and attractive (real) form

$$
\Psi^{\prime}=\frac{\sqrt{2}}{c} \sigma^{0 \prime} \bar{\sigma}^{0 \prime}+\frac{\sqrt{2} k}{c} \phi_{2}^{0} \bar{\phi}_{2}^{0} .
$$

Remark 12. The Bondi mass, $M_{\mathrm{B}}=-\frac{c^{2}}{2 \sqrt{2} G} \Psi^{0}$, and the original mass of the Reissner-Nordström (Schwarzschild) unperturbed metric, $M_{\mathrm{RN}}=-\frac{c^{2}}{2 \sqrt{2} G} \psi_{2}^{00}$, i.e., the $l=0$ harmonic of $\psi_{2}^{0}$, differ by a quadratic term in the shear, the $l=0$ part of $\sigma \dot{\bar{\sigma}}$. This suggests that the observed mass of an object is partially determined by its time-dependent quadrupole moment. 
Upon extracting the $l=0$ harmonic portion of Eq. (6.51) as well as inserting our various physical identifications for the objects involved, we obtain the Bondi mass loss theorem:

$$
\begin{aligned}
M_{\mathrm{B}}^{\prime}= & -\frac{G}{5 c^{7}}\left(Q_{\text {Mass }}^{j k \prime \prime} Q_{\text {Mass }}^{j k \prime \prime}+Q_{\mathrm{Spin}}^{j k \prime \prime \prime} Q_{\mathrm{Spin}}^{j k \prime \prime \prime}\right)-\frac{4 q^{2}}{3 c^{5}}\left(\eta_{E}^{k \prime \prime} \eta_{E}^{k \prime \prime}+\eta_{M}^{k \prime \prime} \eta_{M}^{k \prime \prime}\right) \\
& -\frac{4}{45 c^{7}}\left(Q_{E}^{j k \prime \prime \prime} Q_{E}^{j k \prime \prime \prime}+Q_{M}^{j k \prime \prime \prime} Q_{M}^{j k \prime \prime \prime}\right) .
\end{aligned}
$$

This mass/energy loss equation contains the classical energy loss due to electric and magnetic dipole radiation and electric and magnetic quadrupole $\left(Q_{E}^{i j}, Q_{M}^{i j}\right)$ radiation. (Note that agreement with the physical quadrupole radiation is recovered after making the aforementioned rescaling $Q_{\mathbb{C}}^{i j} \rightarrow 2 \sqrt{2} Q_{\mathbb{C}}^{i j}$.) The gravitational energy loss is the conventional quadrupole loss by the identification (6.36) of $\xi^{i j}$ with the gravitational quadrupole moment $Q_{\text {Grav }}^{i j}$.

The momentum loss equation, from the $l=1$ part of Eq. (6.51), is then identified with the recoil force due to momentum radiation:

$$
P^{i \prime}=F_{\text {recoil }}^{i},
$$

where

$$
\begin{aligned}
F_{\text {recoil }}^{i}= & \frac{2 G}{15 c^{6}}\left(Q_{\text {Spin }}^{j l \prime \prime \prime} Q_{\text {Mass }}^{k j \prime \prime \prime}-Q_{\text {Mass }}^{j l \prime \prime \prime} Q_{\text {Spin }}^{k j \prime \prime \prime}\right) \epsilon_{k l i}+\frac{2 q^{2}}{3 c^{4}}\left(\eta_{R}^{j \prime \prime} \eta_{I}^{k \prime \prime}-\eta_{I}^{j \prime \prime} \eta_{R}^{k \prime \prime}\right) \epsilon_{k j i} \\
& -\frac{4 \sqrt{2} q}{15 c^{5}}\left(\eta_{R}^{j \prime \prime} Q_{E}^{j i \prime \prime \prime}+\eta_{I}^{j \prime \prime} Q_{M}^{j i \prime \prime \prime}\right)-\frac{4}{135 c^{6}}\left(Q_{M}^{j l \prime \prime \prime} Q_{E}^{k j \prime \prime \prime}-Q_{E}^{j l \prime \prime \prime} Q_{M}^{k j \prime \prime \prime}\right) .
\end{aligned}
$$

Finally, we can substitute in the $P^{i}$ from Eq. (6.49) to obtain Newton's second law of motion:

$$
M_{\mathrm{B}} \xi_{R}^{i \prime \prime}=F^{i},
$$

with

$$
\begin{aligned}
F^{i} & =-M_{\mathrm{B}}^{\prime} \xi_{R}^{i \prime}+\frac{2}{3} c^{-3} q^{2} \eta_{R}^{i \prime \prime \prime}+F_{\text {recoil }}^{i}-\Xi^{i \prime}, \\
\Xi & =\mathfrak{P}_{1}+\mathfrak{P}_{2}+\mathfrak{P}_{3}
\end{aligned}
$$

\section{Physical Content}

There are several things to observe and comment on concerning Eqs. (6.54) and (6.55):

- If the complex world line associated with the Maxwell center of charge coincides with the complex center of mass, i.e., if $\eta^{i}=\xi^{i}$, the term

$$
\frac{2}{3} c^{-3} q^{2} \xi_{R}^{i \prime \prime \prime}
$$

becomes the classical electrodynamic radiation reaction force.

- This result follows directly from the Einstein-Maxwell equations. There was no model building other than requiring that the two complex world lines coincide. Furthermore, there was no mass renormalization; the mass was simply the conventional Bondi mass as seen at infinity. The problem of the runaway solutions, though not solved here, is converted to the stability of the Einstein-Maxwell equations with the 'coinciding' condition on the two world lines. If the two world lines do not coincide, i.e., the Maxwell world line forms independent data, then there is no problem of unstable behavior. This suggests a resolution to the problem of the unstable solutions: one should treat the source as a structured object, not a point, and centers of mass and charge as independent quantities. 
- The $F_{\text {recoil }}^{i}$ is the recoil force from momentum radiation.

- The $\Xi^{i \prime}=-F_{\mathrm{RR}}^{i}$ can be interpreted as the gravitational radiation reaction.

- The first term in $F^{i}$, i.e., $-M_{\mathrm{B}}^{\prime} \xi_{R}^{i \prime}$, is identical to a term in the classical Lorentz-Dirac equations of motion. Again it is nice to see it appearing, but with the use of the mass loss equation it is in reality third order.

\subsection{Other related results}

The ideas involved in the identification, at future null infinity, of interior physical quantities that were developed in the proceeding sections can also be applied to a variety of different perturbation schemes. Bramson, Adamo and Newman [19, 2, 4] have investigated how gravitational perturbations originating solely from a Maxwell radiation field can be carried through again using the asymptotic Bianchi identities to obtain, in a different context, the same identifications: a complex center-of-mass/charge world line, energy and momentum loss, as well as an angular momentum flux law that agrees exactly with the predictions of classical electromagnetic field theory. This scheme yields (up to the order of the perturbation) an approximation for the metric in the interior of the perturbed spacetime.

We briefly describe this procedure. One initially chooses as a background an exact solution of the Einstein equations; three cases were studied: flat Minkowski spacetime, the Schwarzschild spacetime with a 'small' mass and the Schwarzschild spacetime with a finite, 'zero order', mass. For such backgrounds, the set of spin coefficients is known and fixed. On this background the Maxwell equations were integrated to obtain the desired electromagnetic field that acts as the gravitational perturbation. Bramson has done this for a pure electric dipole solution [19, 2] on the Minkowski background. Recent work has used an electric and magnetic dipole field with a Coulomb charge [4]. The resulting Maxwell field, in each case, is then inserted into the asymptotic Bianchi identities, which, in turn, determine the behavior of the perturbed asymptotic Weyl tensor, i.e., the Maxwell field induces nontrivial changes to the gravitational field. Treating the Maxwell field as first order, the calculations were done to second order, as was done earlier in this review.

Using the just obtained Weyl tensor terms, one can proceed to the integration of the spincoefficient equations and the second-order metric tensor. For example, one finds that the dipole Maxwell field induces a second-order Bondi shear, $\sigma^{0}$. (This in principle would lead to a fourthorder gravitational energy loss, which in our approximation is ignored.)

Returning to the point of view of this section, the perturbed Weyl tensor can now be used to obtain the same physical identifications described earlier, i.e., by employing a null rotation to set $\psi_{1}^{0 * i}=0$, equations of motion and asymptotic physical quantities, (e.g., center of mass and charge, kinematic expressions for momentum and angular momentum, etc.) for the interior of the system could be found. Although we will not repeat these calculations here, we present a few of the results. Though the calculations are similar to the earlier ones, they differ in two ways: there is no first-order freely given Bondi shear and the perturbation term orders are different.

For instance, the perturbations induced by a Coulomb charge and general electromagnetic dipole Maxwell field in a Schwarzschild background lead to energy, momentum, and angular momentum flux relations [4]:

$$
\begin{aligned}
M_{\mathrm{B}}^{\prime} & =-\frac{2}{3 c^{5}}\left(D_{E}^{i \prime \prime} D_{E}^{i \prime \prime}+D_{M}^{i \prime \prime} D_{M}^{i \prime \prime}\right), \\
P^{i \prime} & =\frac{1}{3 c^{4}} D_{E}^{k \prime \prime} D_{M}^{j \prime \prime} \epsilon_{k j i}, \\
J^{k \prime} & =\frac{2}{3 c^{3}}\left(D_{E}^{i \prime \prime} D_{E}^{j \prime}+D_{M}^{i \prime \prime} D_{M}^{j \prime}\right) \epsilon_{i j k},
\end{aligned}
$$


all of which agree exactly with predictions from classical field theory [43].

The familiarity of such results is an exhibit in favor of the physical identification methods described in this review, i.e., they are a confirmation of the consistency of the identification scheme.

\section{Gauge (BMS) Invariance}

The issue of gauge invariance, the understanding of which is not obvious or easy, must now be addressed. The claim is that the work described here is in fact gauge (or BMS) invariant.

First of all we have, $\mathfrak{I}_{\mathbb{C}}^{+}$, or its real part, $\mathfrak{I}^{+}$. On $\mathfrak{I}_{\mathbb{C}}^{+}$, for each choice of spacetime interior and solution of the Einstein-Maxwell equations, we have its UCF, either in its complex version, $u_{\mathrm{B}}=X(\tau, \zeta, \tilde{\zeta})$, or its real version, Eq. (6.21). The geometric picture of the UCF is a one-parameter family of slicings (complex or real) of $\mathfrak{I}_{\mathbb{C}}^{+}$or $\mathfrak{I}^{+}$. This is a geometric construct that has a different appearance or description in different Bondi coordinate systems. It is this difference that we must investigate. We concentrate on the complex version.

Under the action of the supertranslation, Eq. (2.63), we have:

$$
X(\tau, \zeta, \tilde{\zeta}) \rightarrow X^{*}(\tau, \zeta, \tilde{\zeta})=X(\tau, \zeta, \tilde{\zeta})+\alpha(\zeta, \tilde{\zeta}),
$$

with $\alpha(\zeta, \tilde{\zeta})$ an arbitrary complex smooth function on (complexified) $S^{2}$. Its effect is to add on a constant to each spherical harmonic coefficient of $X$. The special case of translations, with

$$
\alpha(\zeta, \tilde{\zeta})=d^{a} \hat{l}_{a}(\zeta, \tilde{\zeta})
$$

simply adds to the $l=(0,1)$ harmonic components the complex constants $d^{a}$, so, via Eq. (6.1), we have the (complex) Poincaré translations,

$$
\xi^{a} \rightarrow \xi^{a *}=\xi^{a}+d^{a} .
$$

The action of the homogeneous Lorentz transformations, Eq. (2.64),

$$
\begin{aligned}
u_{\mathrm{B}}^{*} & =K u_{\mathrm{B}}, \\
K & =\frac{1+\zeta \bar{\zeta}}{(a \zeta+b)(\bar{a} \bar{\zeta}+\bar{b})+(c \zeta+d)(\bar{c} \bar{\zeta}+\bar{d})}, \\
\zeta^{*} & =\frac{a \zeta+b}{c \zeta+d} ; \quad a d-b c=1 . \\
e^{i \lambda} & =\frac{c \zeta+d}{\bar{c} \bar{\zeta}+\bar{d}}
\end{aligned}
$$

is considerably more complicated. It leads to

$$
X^{*}\left(\tau, \zeta^{*}, \tilde{\zeta}^{*}\right)=K X(\tau, \zeta, \tilde{\zeta}) .
$$

Before discussing the relevant effects of the Lorentz transformations on our considerations we first digress and describe an important technical issue concerning representation of the homogeneous Lorentz group.

The representation theory of the Lorentz group, developed and described by Gelfand, Graev and Vilenkin [27] used homogeneous functions of two complex variables (homogeneous of degrees, $n_{1}-1$ and $\left.n_{2}-1\right)$ as the representation space. Here we summarize these ideas via an equivalent method [33, 26] using spin-weighted functions on the sphere as the representation spaces. In the notation of Gelfand, Graev and Vilenkin, representations are labeled by the two numbers $\left(n_{1}, n_{2}\right)$ or by $(s, w)$, with $\left(n_{1}, n_{2}\right)=(w-s+1, w+s+1)$. The ' $s$ ' is the same ' $s$ ' as in the spin weighted 
functions and ' $w$ ' is the conformal weight [60] (sometimes called 'boost weight'). The different representations are written as $D_{\left(n_{1}, n_{2}\right)}$. The special case of irreducible unitary representations, which occur when $\left(n_{1}, n_{2}\right)$ are not integers, plays no role for us and will not be discussed. We consider only the case when $\left(n_{1}, n_{2}\right)$ are integers so that the $(s, w)$ take integer or half integer values. If $n_{1}$ and $n_{2}$ are both positive integers or both negative integers, we have, respectively, the positive or negative integer representations. The representation space, for each $(s, w)$, are the functions on the sphere, $\eta_{(s, w)}(\zeta, \bar{\zeta})$, that can be expanded in spin-weighted spherical harmonics, ${ }_{s} Y_{l m}(\zeta, \bar{\zeta})$, so that

$$
\eta_{(s, w)}(\zeta, \bar{\zeta})=\sum_{l=s}^{\infty} \eta_{(l m) s} Y_{l m}(\zeta, \bar{\zeta})
$$

Under the action of the Lorentz group (7.4) - (7.5), they transform as

$$
\widehat{\eta}_{(s, w)}(\widehat{\zeta}, \bar{\zeta})=e^{i s \lambda} K^{w} \eta_{(s, w)}(\zeta, \bar{\zeta})
$$

These representations, in general, are neither irreducible nor totally reducible. For us the important point is that many of these representations possess an invariant finite-dimensional subspace which (often) corresponds to the usual finite dimensional tensor representation space. Under the transformation, Eq. (7.8), the finite number of coefficients in these subspaces transform among themselves. It is this fact which we heavily utilize. More specifically we have two related situations: (1) when the $\left(n_{1}, n_{2}\right)$ are both positive integers (or $\left.w \geq|s|\right)$ there will be finite dimensional invariant subspaces, $D_{\left(n_{1}, n_{2}\right)}^{+}$, which are spanned by the basis vectors ${ }_{s} Y_{l m}(\zeta, \bar{\zeta})$, with $l$ given in the range, $|s| \leq l \leq w$. All the finite dimensional representations can be obtained in this manner. And (2) when the $\left(-n_{1},-n_{2}\right)$ are both negative integers (i.e., we have a negative integer representation) there will be an infinite dimensional invariant subspace, $D_{\left(-n_{1},-n_{2}\right)}^{-}$, described elsewhere [33]. One, however, can obtain a finite dimensional representation for each negative integer case by the following construction: One forms the factor space, $D_{\left(-n_{1},-n_{2}\right)} / D_{\left(-n_{1},-n_{2}\right)}^{-}$. This space is isomorphic to one of the finite dimensional spaces associated with the positive integers. The explicit form of the isomorphism, which is not needed here, is given in Held et al. [33, 27].

Of major interest for us is not so much the invariant subspaces but instead their interactions with their compliments (the full vector space modulo the invariant subspace). Under the action of the Lorentz transformations applied to a general vector in the representation space, the components of the invariant subspaces remain in the invariant subspace but in addition components of the complement move into the invariant subspace. On the other hand, the components of the invariant subspaces do not move into the complement subspace: the transformed components of the compliment involve only the original compliment components. The transformation thus has a nontrivial Jordan form.

Rather than give the full description of these invariant subspaces we confine ourselves to the few cases of relevance to us.

\section{The Good-Cut Function}

Though our interest is primarily in the negative integer representations, we first address the positive integer case of the $s=0$ and $w=1,\left[\left(n_{1}, n_{2}\right)=(2,2)\right]$, representation. The harmonics, $l=(0,1)$ form the invariant subspace. The cut function, $X(\tau, \zeta, \bar{\zeta})$, for each fix values of $\tau$, lies in this space.

We write the GCF as

$$
\begin{aligned}
u_{\mathrm{B}} & =X\left(\xi^{a}(\tau), \zeta, \bar{\zeta}\right), \\
& =\xi^{a} \hat{l}_{a}(\zeta, \bar{\zeta})+\sum_{l,|m| \leq l} H^{l m}\left(\xi^{a}\right) Y_{l m}(\zeta, \bar{\zeta}) .
\end{aligned}
$$


After the Lorentz transformation, the geometric slicings have not changed but their description in terms of $\left(u_{\mathrm{B}}, \zeta, \bar{\zeta}\right)$ has changed to that of $\left(u_{\mathrm{B}}^{*}, \zeta^{*}, \bar{\zeta}^{*}\right)$. This leads to

$$
\begin{aligned}
u_{\mathrm{B}}^{*} & =K X=X^{*}, \\
& =\xi^{a *} \hat{l}_{a}^{*}\left(\zeta^{*}, \bar{\zeta}^{*}\right)+\sum_{l,|m| \leq l} H^{l m *}\left(\xi^{a *}\right) Y_{l m}\left(\zeta^{*}, \bar{\zeta}^{*}\right) .
\end{aligned}
$$

Using the transformation properties of the invariant subspace and its compliment we see that the coordinate transformation must have the form:

$$
\xi^{a *}=F^{a}\left(\xi^{b}, H^{l m}\left(\xi^{b}\right), \ldots\right),
$$

in other words it moves the higher harmonic coefficients down to the $l=(0,1)$ coefficients. The higher harmonic coefficients transform among themselves;

$$
H^{l m *}\left(\xi^{a *}\right)=F^{l m}\left(\ldots, H^{l^{*} m^{*}}\left(\xi^{b}\right), \ldots\right) .
$$

Treating the $\xi^{a}$ and $\xi^{a *}$ as functions of $\tau$, we have

$$
V=\partial_{\tau} X=v^{a} \frac{\partial X}{\partial \xi^{a}}=v^{a} \hat{l}_{a}+v^{a} \sum_{l,|m| \leq l} \frac{\partial H^{l m}\left(\xi^{b}\right)}{\partial \xi^{a}} Y_{l m}(\zeta, \bar{\zeta})
$$

where

$$
v^{a}=\frac{d \xi^{a}}{d \tau}, \quad v^{a *}=\frac{\partial F^{a}}{\partial \xi^{b}} \frac{d \xi^{b}}{d \tau}=F_{, b}^{a} v^{b}
$$

It then follows that $V$ transforms as

$$
\begin{aligned}
V^{*}=K V & =v^{a *}\left(\hat{l}_{a}^{*}+\sum_{l,|m| \leq l} H^{l m *},{ }_{a} Y_{l m}\left(\zeta^{*}, \bar{\zeta}^{*}\right)\right) \\
& =v^{b} F^{a}{ }_{, b}\left(\hat{l}_{a}^{*}+\sum_{l,|m| \leq l} H^{l m *},{ }_{a} Y_{l m}\left(\zeta^{*}, \bar{\zeta}^{*}\right)\right) .
\end{aligned}
$$

Our $\mathcal{H}$-space coordinates, $z^{a}=\xi^{a}$, and their $\tau$-derivatives, $v^{a}$, are the $l=(0,1)$ coefficients of harmonic expansions of the $X$ and $V$ respectively. We have shown that a Lorentz transformation induces a specific coordinate transformation (and associated vector transformation) on these coefficients.

\section{The Mass Aspect}

A second important example concerns the Bondi mass aspect, (where we have introduced the $Y_{0}^{0}$ for simplicity of treatment of numerical factors)

$$
\Psi \equiv \Psi_{(0,-3)}=\Psi^{0} Y_{0}^{0}+\Psi^{i} Y_{1 i}^{0}+\Psi^{i j} Y_{2 i j}^{0}+\ldots
$$

This is a $s=0$ and $w=-3,\left[\left(n_{1}, n_{2}\right)=(-2,-2)\right]$ quantity. The invariant subspace has a basis set of the harmonics with $l \geq 2$. The factor space is isomorphic to the finite dimensional positive integer space $\left(n_{1}, n_{2}\right)=(2,2)$ and hence the harmonic coefficients of $l=(0,1)$ lie in this finite dimensional representation space. From this isomorphism we know that functions of the form, $\Psi^{0} Y_{0}^{0}+\Psi^{i} Y_{1 i}^{0}$, whose four coefficients are proportional to the Bondi four-momentum

$$
P^{a}=\left(M c, P^{i}\right),
$$


form a Lorentzian four-vector. Note that we have rescaled the $\Psi^{0}$ in Eq. (7.14) by the $Y_{0}^{0}$, differing from that of Eq. (2.59) in order to give the spherical harmonic coefficients immediate physical meaning without the use of the factors in equations Eq. (2.60) and Eq. (2.61).

This is the justification for calling the $l=(0,1)$ harmonics of the mass aspect a Lorentzian four-vector. (Technically, the Bondi four-momentum is a co-factor but we have allowed ourselves a slight notational irregularity.)

\section{The Complex Dipole Moment}

The Weyl tensor component, $\psi_{1}^{0}$, has $s=1$ and $w=-3,\left[\left(n_{1}, n_{2}\right)=(-3,-1)\right]$. The associated finite dimensional factor space is isomorphic to the finite part of the $s=-1, w=1,\left[\left(n_{1}, n_{2}\right)=(3,1)\right]$ representation. We have that

$$
\psi_{1}^{0} \equiv \psi_{1(1,-3)}^{0}=\psi_{1}^{0 i} Y_{1 i}^{1}+\psi_{1}^{0 i j} Y_{2 i j}^{1}+\ldots
$$

leads to the finite-dimensional representation space

$$
\text { finite space }=\operatorname{span}\left\{\psi_{1 i}^{0} Y_{1 i}^{-1}\right\} .
$$

What finite tensor transformation this corresponds to is a slightly more complicated question than that of the previous examples of Lorentzian vectors. In fact, it corresponds to the Lorentz transformations applied to (complex) self-dual antisymmetric two-index tensors [42]. We clarify this with an example from Maxwell theory: from a given $\mathbf{E}$ and $\mathbf{B}$, the Maxwell tensor, $F^{a b}$, and then its self-dual version can be constructed:

$$
W^{a b+}=F^{a b}+i F^{* a b}
$$

A Lorentz transformation applied to the tensor, $W^{a b+}$, is equivalent [43] to the same transformation applied to

$$
\psi_{1}^{0}{ }^{i} \leftrightarrow(\mathbf{E}+i \mathbf{B})^{i} .
$$

This allows us to assign Lorentzian invariant physical meaning to our identifications of the complex mass dipole moment and angular momentum vector, $D_{\text {(mass) }}^{i}+i J^{i}$.

\section{General Invariants}

Our last example is a general discussion of how to construct Lorentzian invariants from the representation spaces. Though we will confine our remarks to just the cases of $s=0$, it is easy to extend them to nonvanishing $s$ by having the two functions have respectively spin-weight $s$ and $-s$.

Consider pairs of conformally weighted functions $(s=0), W_{(w)}, G_{(-w-2)}$, with weights respectively, $(w,-w-2)$. They are considered to be in dual spaces. Our claim is that the integrals of the form

$$
I=\int G_{(-w-2)} W_{(w)} d \Omega
$$

are Lorentz invariants.

We first point out that under the fractional linear transformation, $\zeta^{*} \leftrightarrow \zeta$, given by Eq. (7.5), the area element on the sphere

$$
d \Omega=\frac{4 i d \zeta \wedge d \bar{\zeta}}{(1+\zeta \bar{\zeta})^{2}}
$$

transforms as [33]

$$
d \Omega^{*}=K^{2} d \Omega .
$$


This leads immediately to

$$
\begin{aligned}
I & =\int G_{(-w-2)}^{*} W_{(w)}^{*} d \Omega^{*} \\
& =\int K^{-w-2} G_{(-w-2)} K^{w} W_{(w)} K^{2} d \Omega \\
& =\int G_{(-w-2)} W_{(w)} d \Omega
\end{aligned}
$$

the claimed result.

There are several immediate simple applications of Eq. (7.19). By choosing an arbitrary $w=$ $-2, s=0$ function, say $G_{(-2)}(\zeta, \bar{\zeta})$ and $W_{(0)}=1$, we immediately have a Lorentzian scalar,

$$
N \equiv \int G_{(-2)} d \Omega=\int G_{(-2)}^{\prime} d \Omega^{\prime}
$$

If this is made more specific by choosing $G_{(-2)}=V^{-2}$, we have the remarkable result (proved in Appendix D) that this scalar yields the $\mathcal{H}$-space metric norm of the "velocity" $v^{a}$, via

$$
8 \pi\left(g_{a b} v^{a} v^{b}\right)^{-1} \equiv \int V^{-2} d \Omega
$$

A simple variant of this arises by taking the derivative of Eq. (7.24) with respect to $v^{a}$, and multiplying by an arbitrary vector, $w^{a}$ leading to

$$
8 \pi w^{a} v_{a}\left(g_{c d} v^{c} v^{d}\right)^{-2} \equiv \int V^{-3} w^{a} Z,_{a} d \Omega .
$$

Many other versions can easily be found. 


\section{Discussion/Conclusion}

\subsection{History/background}

The work reported in this document has had a very long gestation period. It began in 1965 [53] with the publication of a paper where a complex coordinate transformation was performed on the Schwarzschild/Reissner-Nordström solutions. This, in a precise sense, moved the 'point source' onto a complex world line in a complexified spacetime. It thereby led to a derivation of the spinning and the charged-spinning particle metrics. How and why this procedure worked was considered to be rather mysterious and a great deal of effort by a variety of people went into trying to unravel it. In the end, the use of the complex coordinate transformation for the derivation of these metrics appeared as if it was simply an accident; that is, a trick with no immediate significance. Nevertheless, the idea of a complex world line, appearing in a natural manner, was an intriguing thought, which frequently returned. Some years later, working on an apparently unrelated subject, we studied and found the condition for a regular NGC, in asymptotically-flat spacetime, to have a vanishing asymptotic shear [12]. This led to the realization that a regular NGC was generated by a complex world line, though originally there was no relationship between the two complex world lines. This condition (our previously discussed shear-free condition, Eq. (4.12)) was eventually shown to be closely related to Penrose's asymptotic twistor theory, and in the flat-space case it led to the Kerr theorem and totally shear-free NGCs. From a very different point of view, searching for asymptotically shear-free complex null surfaces, the good-cut equation was found with its fourcomplex parameter solution space, leading to the theory of $\mathcal{H}$-space.

Years later, the different strands came together. The shear-free condition was found to be closely related to the good-cut equation; namely, that one equation could be transformed into the other. The major surprise came when we discovered that the regular solutions of either equation were generated by complex world lines in an auxiliary space [39]. These complex world lines were interpreted as being complex analytic curves in the associated $\mathcal{H}$-space. The deeper meaning of this remains a major question still to be fully resolved; it is this issue which is partially addressed in the present work.

At first, these complex world lines were associated with the spinning, charged and uncharged particle metrics - type D algebraically special metrics, but now can be seen as just special cases of the asymptotically flat solutions. Since these metrics were algebraically special, among the many possible asymptotically shear-free NGCs there was (at least) one totally shear-free (rather than asymptotically shear-free) congruence coming from the Goldberg-Sachs theorem. Their associated world lines were the ones first discovered in 1965 (coming, by accident, from the complex coordinate transformation), and became the complex center-of-mass world line (which coincided with the complex center of charge in the charged case.). This observation was the clue for how to search for the generalization of the special world line associated with algebraically-special metrics and thus, in general, how to look for the special world line (and congruence) to be identified with the complex center of mass for arbitrary asymptotically flat spacetimes.

For the algebraically-special metrics, the null tetrad system at $\mathfrak{I}^{+}$with one leg being the tangent null vector to the shear-free congruence leads to the vanishing of the asymptotic Weyl tensor component, i.e., $\psi_{0}^{*}=\psi_{1}^{*}=0$. For the general case, no tetrad exists with that property but one can always find a null tetrad with one leg being tangent to an asymptotically shear-free congruence so that the $l=1$ harmonics of $\psi_{1}^{0 *}$ vanish. It is precisely that choice of tetrad that led to our definition of the complex center of mass.

\subsection{Other choices for physical identification}

The question of whether our definition of the complex center of mass is the best possible definition, or even a reasonable one, is not easy to answer. We did try to establish a criteria for choosing 
such a definition: (i) it should predict already known physical laws or reasonable new laws, (ii) it should have a clear geometric foundation and a logical consistency and (iii) it should agree with special cases, mainly the algebraically-special metrics or analogies with flat-space Maxwell theory. We did try out several other possible choices [42] and found them all failing. This clearly does not rule out others that we did not think of, but at the present our choice appears to be both natural and effective in making contact with physical phenomena. There still remains the mystery of the physical meaning of $\mathcal{H}$-space or why it leads to such reasonable physical results. Possible resolutions appear to lie in the duality between the complex world lines and real but twisting NGCs [8].

\subsection{Predictions}

Our equations of motion are simultaneously satisfactory and unsatisfactory: they yield the equations of motion for an isolated object with a great deal of internal structure (time-dependent multipoles with the emission of gravitational and electromagnetic radiation) in the form of Newton's second law. In addition, they contain a definition of angular momentum with an angular momentum flux. The dipole part of the angular momentum flux agrees with classical electromagnetic theory. Unfortunately, there appears to be no immediate way to study or describe interacting particles in this manner.

However, there are some areas where these ideas might be tested, though the effects would be very small. For example, earlier we saw that there was a contribution to the Bondi mass (an addition to the Reissner-Nordström mass) from the quadrupole moment,

$$
M_{\mathrm{B}}-M_{R N} \equiv \Delta M=-\frac{1}{5} G c^{-6} \operatorname{Re} Q_{\mathrm{Grav}}^{i j \prime} \bar{Q}_{\mathrm{Grav}}^{i j \prime \prime \prime} .
$$

There were predicted contributions to both the momentum and angular momentum flux from the gravitational and electromagnetic quadrupole radiation as well as new terms in the angular momentum flux equation, i.e., the charge/magnetic-dipole coupling term

$$
\frac{2}{3} q^{2} c^{-2} \eta_{I}^{i \prime}=\frac{2}{3} q c^{-2} D_{M}^{i \prime}
$$

This interpretation of this term is slightly ambiguous in that it could be identified, in the flux equation, as either a contribution to the angular momentum flux (as we are suggesting now) or as a contribution to the angular momentum itself. See Eq. (6.46).

There are other unfamiliar terms that can be thought of as predictions of this theoretical construct, though how to possibly measure them is not at all clear.

\subsection{Summary of results}

1. From the asymptotic Weyl and Maxwell tensors, with their transformation properties, we were able (via the asymptotically shear-free NGC) to obtain two complex world lines - a complex 'center of mass' and 'complex center of charge' in the auxiliary $\mathcal{H}$-space. When 'viewed' from a Bondi coordinate and tetrad system, this led to an expression for the real center of mass of the gravitating system and a kinematic expression for the total angular momentum (including intrinsic spin and orbital angular momentum), as seen from null infinity. It is interesting to observe that the kinematical expressions for the classical linear momentum and angular momentum came directly from the gravitational dynamical laws (Bianchi identities) for the evolution of the Weyl tensor.

2. From the real parts of one of the asymptotic Bianchi identities, Eq. (2.52), we found the standard kinematic expression for the Bondi linear momentum, $P=M \xi_{R}^{\prime}+\ldots$, with the 
radiation reaction term $\frac{2 q^{2}}{3 c^{3}} v_{R}^{k \prime}$ among others. The imaginary part was the angular momentum conservation law with a very natural looking flux expression of the form:

$$
J^{\prime}=\text { Flux }_{\mathrm{E} \& M} \text { dipole }+ \text { Flux }_{\text {Grav }}+\text { Flux }_{\mathrm{E} \& M \text { quad }} .
$$

The first flux term is identical to that calculated from classical electromagnetic theory

3. Using the kinematic expression for the Bondi momentum in a second Bianchi identity (2.53), we obtained a second-order ODE for the center of mass world line that could be identified with Newton's second law with radiation reaction forces and recoil forces, $M_{\mathrm{B}} \xi_{R}^{\prime \prime}=F$.

4. From Bondi's mass/energy loss theorem we obtained the correct energy flux from the electromagnetic dipole and quadrupole radiation as well as the gravitational quadrupole radiation.

5. From the specialized case where the two world lines coincide and the definitions of spin and magnetic moment, we obtained the Dirac gyromagnetic ratio, $g=2$. In addition, we find the classical electrodynamic radiation reaction term with the correct numerical factors. In this case we have the identifications of the meaning of the complex position vector: $\xi^{i}=\xi_{R}^{i}+i \xi_{I}^{i}$.

$$
\begin{aligned}
\xi_{R}^{i} & =\text { center-of-mass position } \\
S^{i} & =M c \xi_{I}^{i}=\text { spin angular momentum } \\
D_{E}^{i} & =q \xi_{R}^{i}=\text { electric dipole moment } \\
D_{M}^{i} & =q \xi_{I}^{i}=\text { magnetic dipole moment }
\end{aligned}
$$

Remark 13. In the past, most discussions of angular momentum make use of group theoretical ideas with Noether theorem type arguments, via the BMS group and the Lorentz subgroup, to define angular momentum. Unfortunately this has been beset with certain difficulties; different authors get slightly different numerical factors in their definitions, with further ambiguities arising from the supertranslation freedom of the BMS group. (See the discussion after Eq. (2.62)) Our approach is very different from the group theoretical approach in that we come to angular momentum directly from the dynamics of the Einstein equations (the asymptotic Bianchi Identities). We use the angular momentum definition from linear theory, Eq. (2.62), (agreed to by virtually all) and then supplement it via conservation equations (the flux law) obtained directly from the Bianchi Identities. We have a unique one-parameter family of cuts coming from the complex world line defining the complex center of mass. This is a geometric structure with no ambiguities. However, another ambiguity does arise by asking which Bondi frame should be used in the description of angular momentum; this is the ambiguity of what coordinate system to use.

\subsection{Issues and open questions}

1. A particularly interesting issue raised by our equations is that of the run-away (unstable) behavior of the equations of motion for a charged particle (with or without an external field). We saw in Eq. (6.54) that there was a driving term in the equation of motion depending on the electric dipole moment (or the real center of charge). This driving term was totally independent of the real center of mass and thus does not lead to the classical instability. However, if we restrict the complex center of charge to be the same as the complex center of mass (a severe, but very attractive restriction leading to $g=2$ ), then the innocuous driving dipole term becomes the classical radiation reaction term - suggesting instability. (Note that in this coinciding case there was no model building - aside from the coinciding lines - and no mass renormalization.) 
A natural question then is: does this unstable behavior really remain? In other words, is it possible that the large number of extra terms in the gravitational radiation reaction or the momentum recoil force might stabilize the situation? Answering this question is extremely difficult. If the gravitational effects do not stabilize, then - at least in this special case the Einstein-Maxwell equations are unstable, since the run-away behavior would lead to an infinite amount of electromagnetic dipole energy loss.

An alternative possible resolution to the classical run-away problem is simply to say that the classical electrodynamic model is wrong; and that one must treat the center of charge as different from the center of mass with its own dynamics.

2. In our approximations, it was assumed that the complex world line yielded cuts of $\mathfrak{I}^{+}$that were close to Bondi cuts. At the present we do not have any straightforward means of finding the world lines and their associated cuts of $\mathfrak{I}^{+}$that are far from the Bondi cuts.

3. As mentioned earlier, when the gravitational and electromagnetic world lines coincide we find the rather surprising result of the Dirac value for the gyromagnetic ratio. Though this appears to be a significant result, we unfortunately do not have any deeper understanding of it.

4. Is it possible that the complex structures that we have been seeing and using are more than just a technical device to organize ideas, and that they have a deeper significance? One direction to explore this is via Penrose's twistor and asymptotic twistor theory. It is known that much of the material described here is closely related to twistor theory; an example is the fact that asymptotic shear-free NGCs are really a special case of the Kerr theorem, an important application of twistor theory (see Appendix A). This connection is being further explored.

5. With much of the kinematics and dynamics of ordinary classical mechanics sitting in our results, i.e., in classical GR, is it possible that ordinary particle quantization could play a role in understanding quantum gravity? Attempts along this line have been made [25, 15] but, so far, without much success.

6. An interesting issue, not yet fully explored but potentially important, is what more can be said about the $\mathcal{H}$-space structures associated with the special regions (the $\mathcal{H}$-space ribbon of (4.40) that are related to the real cuts of null infinity. We touch on this briefly below.

7. Another issue to be explored comes from the duality between the complex $\mathcal{H}$-space lightcones and the real shear-free but twisting NGCs in the real physical spacetime. From either one the other can be determined. It appears as if one might be able to reinterpret (almost) all the $\mathcal{H}$-space structures in terms of real structures associated with the optical parameters of the twisting NGCs and the real slicings associated with the ribbons. This reinterpretation would likely result in lost geometric simplicity and elegance - but perhaps would avoid the mysterious use of the complex $\mathcal{H}$-space for physical identifications.

8. As a final remark, we want to point out that there is an issue that we have ignored: do the asymptotic solutions of the Einstein equations that we have discussed and used throughout this work really exist? By 'really exist' we mean the following: are there, in sufficiently general circumstances, Cauchy surfaces with physically-given data such that their evolution yields these asymptotic solutions? We have tacitly assumed throughout, with physical justification but no rigorous mathematical justification, that the full interior vacuum Einstein equations do lead to these asymptotic situations. However, there has been a great deal of deep and difficult analysis $[24,20,21]$ showing, in fact, that large classes of solutions to the Cauchy problem 
with physically-relevant data do lead to the asymptotic behavior we have discussed. Recently there has been progress made on the same problem for the Einstein-Maxwell equations.

\subsection{New interpretations and future directions}

Throughout this review, we have focused on classical general relativity as expressed in the NewmanPenrose (or spin-coefficient) formalism; yet despite this highly classical setting, the geometric structures discussed here bear a striking resemblance to ideas from more ambitious areas of theoretical physics. In particular, the relationship between real, twisting asymptotically shear-free NGCs in asymptotically flat spacetime and complex, twist-free NGCs in (complex) $\mathcal{H}$-space appears to form a dual pair. The complexified asymptotic boundary $\mathfrak{I}_{\mathbb{C}}^{+}$acts as the translator between these two descriptions: from $\mathfrak{I}_{\mathbb{C}}^{+}$we determine the complexified congruence in $\mathcal{H}$-space via the angle fields $L$ and $\tilde{L}$ or the real congruence (in the real spacetime) via $L$ and $\bar{L}$. Imposing reality conditions in the former case gives an open world sheet (the ' $\mathcal{H}$-space ribbon' discussed in the text) for the NGC's complex source. In the real case, the twisting congruence's caustic set in Minkowski space (which is interpreted as its real source) forms a closed loop propagating in real time [7], or a closed world sheet. In the asymptotically flat case, we cannot follow twisting congruences back to their real caustic set, and they must be represented by the dual 'ribbon'.

In both cases, we see that the congruence's source is a structure which has a natural interpretation as a classical string, with the boundary $\mathfrak{I}_{\mathbb{C}}^{+}$interpolating between the two descriptions. This is suggestive of the so-called 'holographic principle', which aims to equate a theory in a $d$-dimensional compact space with another theory defined on its $d$-1-dimensional boundary [77, 17]. In practice, this can allow one to interpolate between a 'physical' theory in one space and a 'dual' theory living on its boundary (or vice versa). In our case, the 'physical' information is the real, twisting NGC in the asymptotically flat spacetime; $\mathfrak{I}_{\mathbb{C}}^{+}$acts as a lens into $\mathcal{H}$-space, which serves as the virtual image space where physical data (such as the mass, linear momentum, angular momentum, etc.) is computed by the methods reviewed here. Hence, it is tempting to refer to $\mathfrak{I}_{\mathbb{C}}^{+}$as the 'holographic screen' for some application of the holographic principle to general relativity. The presence of classical string-like structures on both sides of the duality makes such a possibility all the more intriguing.

This should be contrasted with the most well-known instance of the holographic principle: the AdS/CFT correspondence [47, 78, 9]. Here, the AdS-boundary acts as the holographic screen between a type IIB string theory in $\mathrm{AdS}_{5} \times S^{5}$ (the virtual image space) and maximally supersymmetric Yang-Mills theory in real four-dimensional Minkowski spacetime (other versions exist, but all involve some supersymmetry). It is interesting that we appear to be describing an instance of the holographic principle that requires no supersymmetry, although 't Hooft's original work relating the planar limit of gauge theories to string-type theories did not use supersymmetry either [76]. In 't Hooft's work, an extra dimension for string propagation enters the picture due to anomaly cancellation in the same way that the extra dimensions of $\mathrm{AdS}_{5} \times S^{5}$ allow for anomaly cancellation in a full supersymmetric string theory. In our investigations, one can think of the analytic continuation of $\mathfrak{I}^{+}$to $\mathfrak{I}_{\mathbb{C}}^{+}$in the same manner: instead of canceling a (quantum) anomaly, the 'extra dimensions' arising from analytic continuation allow us to reinterpret the real twisting NGC in terms of a simpler geometric structure, namely the complex light-cones in $\mathcal{H}$-space.

It is worth noting that this is not the first time that there has been a suggested connection between structures in asymptotically flat spacetimes and the holographic principle. Most prior studies have attempted to understand such a duality in terms of the BMS group, which serves as the symmetry group of the asymptotic boundary [10]. Loosely speaking, these studies take their cue more directly from the AdS/CFT correspondence: by studying fields living on $\mathfrak{I}^{+}$which carry representations of the BMS group, one hopes to reconstruct the full interior of the spacetime 'holographically'. It would be interesting to see how, if at all, our methodology relates to this 
program of research.

Additionally, as we have mentioned throughout this review (and further elaborated in Appendix A), the nature of many of the objects studied here is highly twistorial. This is essentially because $\mathcal{H}$-space is a complex vacuum spacetime equipped with an anti-self-dual metric, and hence possesses a curved twistor space by Penrose's nonlinear graviton construction [32]. It would be interesting to know if our procedure for identifying the complex center of mass (and/or charge) in an asymptotically flat spacetime could be phrased purely in terms of twistor theory. Furthermore, the past several years have seen dramatic progress in using twistor theory to study gauge theories and their scattering amplitudes [1]. These techniques may provide the most promising route for connecting our work with any quantized version of gravity, as illustrated by the recent twistorial derivation of the tree-level MHV graviton scattering amplitude [48].

While the interpretations we have suggested here are far from precise, they suggest a myriad of further directions which research in this area could take. It would be truly fascinating for a topic as old as asymptotically flat spacetimes to make meaningful contact with ambitious new areas of mathematics and physics such as holography or twistorial scattering theory.

\section{Acknowledgments}

We are happy to note and acknowledge the great deal of detailed help and understanding that we have received over the years from Gilberto Silva-Ortigoza, our co-author on many of the earlier papers on the present subject. Many others have contributed to our understanding of asymptoticallyflat spacetimes. Prime among them are Roger Penrose, Andrzej Trautman, Jerzy Lewandowski, Pawel Nurowski, Paul Tod, Lionel Mason, Jörg Frauendiener and Helmut Friedrich. We thank them all.

We also want to point out the very early work of Brian Bramson [18], William Hallidy and Malcolm Ludvigsen [30]. Their ideas, though not fully developed, are close to the ideas developed here, and should be viewed as precursors or preliminary formulations of our theory. We apologize for not having noticed them earlier and giving them more credit. 


\section{A Twistor Theory}

Throughout this review, the study of the asymptotic gravitational field has been at the heart of all our investigations, and there is a natural connection between asymptotic gravitational fields and twistor theory. We give here a brief overview of Penrose's asymptotic twistor theory (see, e.g., $[67,64,35])$ and its connection to the good-cut equation and the study of asymptotically shear-free NGCs at $\mathfrak{I}^{+}$; for a more in depth exposition of this connection, see [51, 6].

Let $\mathcal{M}$ be any asymptotically-flat spacetime manifold, with conformal future null infinity $\mathfrak{I}^{+}$, coordinatized by $\left(u_{\mathrm{B}}, \zeta, \bar{\zeta}\right)$. We can consider the complexification of $\mathfrak{I}^{+}$, referred to as $\mathfrak{I}_{\mathbb{C}}^{+}$, which is in turn coordinatized by $\left(u_{\mathrm{B}}, \zeta, \tilde{\zeta}\right)$, where now $u_{\mathrm{B}} \in \mathbb{C}$ and $\tilde{\zeta}$ is different, but close to $\bar{\zeta}$. Assuming analytic asymptotic Bondi shear $\sigma^{0}\left(u_{\mathrm{B}}, \zeta, \bar{\zeta}\right)$, it can then be analytically continued to $\mathfrak{I}_{\mathbb{C}}^{+}$, i.e., we can consider $\sigma^{0}\left(u_{\mathrm{B}}, \zeta, \tilde{\zeta}\right)$. We have seen in Section 4 that solutions to the good-cut equation

$$
\check{\partial}^{2} G=\sigma^{0}(G, \zeta, \tilde{\zeta})
$$

yield a four complex parameter family of solutions, given by

$$
u_{\mathrm{B}}=Z\left(z^{a} ; \zeta, \tilde{\zeta}\right) .
$$

In our prior discussions, we interpreted these solutions as defining a four (complex) parameter family of surfaces on $\mathfrak{I}_{\mathbb{C}}^{+}$corresponding to each choice of the parameters $z^{a}$. In order to force agreement with the conventional description of Penrose's asymptotic twistor theory we must use the complex conjugate good-cut equation

$$
\overline{\check{\partial}}^{2} \bar{G}=\bar{\sigma}^{0}(\bar{G}, \zeta, \tilde{\zeta}),
$$

whose properties are identical to that of the good-cut equation. Its solutions, written as

$$
u_{\mathrm{B}}=\bar{Z}\left(\bar{z}^{a} ; \zeta, \tilde{\zeta}\right),
$$

define complex two-surfaces in $\mathfrak{I}_{\mathbb{C}}^{+}$for fixed $\bar{z}^{a}$. If we fix $\zeta=\zeta_{0} \in \mathbb{C}$, then Eq. (A.3) becomes an ordinary second-order differential equation with solutions describing curves in $\left(u_{\mathrm{B}}, \tilde{\zeta}\right)$ space. Hence, each solution to this ODE is given by specifying initial conditions for $\tilde{G}$ and $\partial_{\tilde{\zeta}} \tilde{G}$ at some arbitrary initial point, $\tilde{\zeta}=\tilde{\zeta}_{0}$.

Note that it is not necessary that $\tilde{\zeta}_{0}=\bar{\zeta}_{0}$ on $\mathfrak{I}_{\mathbb{C}}^{+}$. However, we chose this initial point to be the complex conjugate of the constant $\zeta_{0}$, i.e., we take $\bar{G}$ and its first $\widetilde{\zeta}$ derivative at $\widetilde{\zeta}=\bar{\zeta}_{0}$ as the initial conditions. Then the initial conditions for Eq. (A.3) can be written as [51]

$$
\begin{aligned}
u_{\mathrm{B} 0} & =\bar{G}\left(\zeta_{0}, \bar{\zeta}_{0}\right), \\
\bar{L}_{0} & =\bar{\partial} G\left(\zeta_{0}, \bar{\zeta}_{0}\right)=P_{0} \frac{\partial \bar{G}}{\partial \bar{\zeta}_{0}}\left(\zeta_{0}, \bar{\zeta}_{0}\right),
\end{aligned}
$$

with $P_{0}=1+\zeta_{0} \tilde{\zeta}_{0}$. Asymptotic projective twistor space, denoted $\mathbb{P} \mathfrak{T}$, is the space of all curves in $\mathfrak{I}_{\mathbb{C}}^{+}$generated by initial condition triplets $\left(u_{\mathrm{B} 0}, \zeta_{0}, \bar{L}_{0}\right)$ [67]: an asymptotic projective twistor is the curve corresponding to $\left(u_{\mathrm{B} 0}, \zeta_{0}, \bar{L}_{0}\right)$. A particular subspace of $\mathbb{P T}$, called null asymptotic projective twistor space $(\mathbb{P N})$, is the family of curves generated by initial conditions, which lie on (real) $\mathfrak{I}^{+}$; that is, at the initial point, $\tilde{\zeta}_{0}=\bar{\zeta}_{0}$, the curve should cross the real $\mathfrak{I}^{+}$, i.e., should be real, $u_{\mathrm{B} 0}=\bar{u}_{\mathrm{B} 0}$. Equivalently, an element of $\mathbb{P} \mathfrak{N}$ can be said to intersect its dual curve (the solution generated by the complex conjugate initial conditions) at $\tilde{\zeta}_{0}=\bar{\zeta}_{0}$. The effect of this is to reduce the three-dimensional complex twistor space to five real dimensions. In standard notation, asymptotic projective twistors are defined in terms of their three complex twistor coordinates, 
$\left(\mu^{0}, \mu^{1}, \zeta\right)[67]$. These twistor coordinates may be re-expressed in terms of the asymptotic twistor curves by

$$
\begin{aligned}
\mu^{0} & =u_{\mathrm{B} 0}-\bar{L}_{0} \bar{\zeta}_{0}, \\
\mu^{1} & =\bar{L}_{0}+\zeta_{0} u_{\mathrm{B} 0}, \\
\zeta & =\zeta_{0} .
\end{aligned}
$$

By only considering the twistor initial conditions $\tilde{\zeta}_{0}=\bar{\zeta}_{0}$, we can drop the initial value notation, and just let $u_{\mathrm{B} 0}=u_{\mathrm{B}}$ and $\tilde{\zeta}=\bar{\zeta}$. The connection of twistor theory with shear-free NGCs takes the form of the flat-space Kerr theorem [67, 51]:

Theorem (Kerr Theorem). Any analytic function on $\mathbb{P T}$ (projective twistor space) generates a shear-free NGC in Minkowski space.

Any analytic function $F\left(\mu^{0}, \mu^{1}, \zeta\right)$ on projective twistor space generates a shear-free NGC in Minkowski space by obtaining the $\bar{L}=\bar{L}\left(u_{\mathrm{B}}, \zeta, \bar{\zeta}\right)$, which defines the congruence via solving the algebraic equation

$$
F\left(\mu^{0}, \mu^{1}, \zeta\right)=F\left(u_{\mathrm{B}}-\overline{L \zeta}, \bar{L}+\zeta u_{\mathrm{B}}, \zeta\right)=0 .
$$

It automatically satisfies the complex conjugate shear-free condition

$$
\bar{\partial} \bar{L}+\dot{\bar{L}}=0 .
$$

We are interested in a version of the Kerr theorem that yields the regular asymptotically shearfree NGCs. Starting with the general four-parameter solution to Eq. (A.3), i.e., $u_{\mathrm{B}}=\bar{Z}\left(\bar{z}^{a} ; \zeta, \tilde{\zeta}\right)$, we chose an arbitrary world line $\bar{z}^{a}=\xi^{a}(\tau)$, so that we have

$$
\begin{aligned}
u_{\mathrm{B}} & =\bar{Z}\left(\xi^{a}(\tau), \zeta, \bar{\zeta}\right)=\bar{G}(\tau, \zeta, \bar{\zeta}), \\
\bar{L}(\tau, \zeta, \bar{\zeta}) & =\bar{\varpi}_{(\tau)} \bar{G}(\tau, \zeta, \bar{\zeta}) .
\end{aligned}
$$

By inserting these into the twistor coordinates, Eq. (A.6), we find

$$
\begin{aligned}
& \mu^{0}(\tau, \zeta, \bar{\zeta})=u_{\mathrm{B}}-\bar{L} \bar{\zeta}=\bar{G}-\bar{\zeta} \bar{\partial}_{(\tau)} \bar{G}, \\
& \mu^{1}(\tau, \zeta, \bar{\zeta})=\bar{L}+\zeta u_{\mathrm{B}}=\bar{\partial}_{(\tau)} \bar{G}+\zeta \bar{G} .
\end{aligned}
$$

The $\mu^{0}$ and $\mu^{1}$ are now functions of $\tau$ and $\zeta$, while the $\bar{\zeta}$ is now to be treated as a fixed quantity, the complex conjugate of $\zeta$, and not as an independent variable. By eliminating $\tau$ in Eqs. (A.8) and (A.9), we obtain a single function of $\mu^{0}, \mu^{1}$, and $\zeta$ : namely, $F\left(\mu^{0}, \mu^{1}, \zeta\right)=0$. Thus, the regular asymptotically shear-free NGCs are described by a special class of twistor functions. This is a special case of a generalized version of the Kerr theorem [67, 51]. 


\section{B CR Structures}

A CR manifold $\mathcal{N}$ is a differentiable manifold endowed with an additional structure called its ' $\mathrm{CR}$ structure'; formally this is a complex distribution (i.e., a sub-bundle $L \subset T \mathcal{N} \otimes \mathbb{C}$ ) which is formally integrable and almost Lagrangian [22]. More concretely, the CR structure can be described by a set of vectors or 1 -forms on $\mathcal{N}$ defined up to a particular gauge freedom. In the context of this review, we are interested in the case where $\mathcal{N}$ is a real three-manifold.

A CR structure on a real three manifold $\mathcal{N}$, with local coordinates $x^{a}$, can be given intrinsically by equivalence classes of one-forms, one real, one complex and its complex conjugate [44]. If we denote the real one-form by $l$ and the complex one-form by $m$, then these are defined up to the transformations:

$$
\begin{aligned}
l & \rightarrow a\left(x^{a}\right) l, \\
m & \rightarrow f\left(x^{a}\right) m+g\left(x^{a}\right) l .
\end{aligned}
$$

The $(a, f, g)$ are functions on $\mathcal{N}: a$ is nonvanishing and real, $f$ and $g$ are complex function with $f$ nonvanishing. We further require that there be a three-fold linear-independence relation between these one-forms [44]:

$$
l \wedge m \wedge \bar{m} \neq 0 .
$$

Any three-manifold with a CR structure is referred to as a three-dimensional CR manifold. There are special classes (referred to as embeddable) of three-dimensional CR manifolds that can be directly embedded into $\mathbb{C}^{2}$. We show how the choice of any specific asymptotically shear-free NGC induces an embeddable CR structure on $\mathfrak{I}^{+}$. Though there are several ways of arriving at this CR structure, the simplest way is to look at the asymptotic null tetrad system associated with the asymptotically shear-free NGC, i.e., look at the $\left(l^{* a}, m^{* a}, \bar{m}^{* a}, n^{* a}\right)$ of Eq. (6.14). The associated dual one-forms, restricted to $\mathfrak{I}^{+}$(after a conformal rescaling of $m$ ), become (with a slight notational dishonesty),

$$
\begin{aligned}
& l^{*}=d u_{\mathrm{B}}-\frac{L}{1+\zeta \bar{\zeta}} d \zeta-\frac{\bar{L}}{1+\zeta \bar{\zeta}} d \bar{\zeta}, \\
& m^{*}=\frac{d \bar{\zeta}}{1+\zeta \bar{\zeta}}, \quad \bar{m}^{*}=\frac{d \zeta}{1+\zeta \bar{\zeta}},
\end{aligned}
$$

with $L=L\left(u_{\mathrm{B}}, \zeta, \bar{\zeta}\right)$, satisfying the shear-free condition. (This same result could have been obtained by manipulating the exterior derivatives of the twistor coordinates, Eq. (A.6).) The dual vectors - also describing the CR structure - are

$$
\begin{aligned}
\overline{\mathfrak{M}} & =P \frac{\partial}{\partial \zeta}+L \frac{\partial}{\partial u_{\mathrm{B}}}=\varlimsup_{\left(u_{B}\right)}+L \frac{\partial}{\partial u_{\mathrm{B}}}, \\
\mathfrak{M} & =P \frac{\partial}{\partial \bar{\zeta}}+\bar{L} \frac{\partial}{\partial u_{\mathrm{B}}}=\varlimsup_{\left(u_{B}\right)}+\bar{L} \frac{\partial}{\partial u_{\mathrm{B}}}, \\
\mathfrak{L} & =\frac{\partial}{\partial u_{\mathrm{B}}} .
\end{aligned}
$$

Therefore, for the situation discussed here, where we have singled out a unique asymptotically shear-free NGC and associated complex world line, we have a uniquely chosen CR structure induced on $\mathfrak{I}^{+}$. To see how our three manifold, $\mathfrak{I}^{+}$, can be embedded into $\mathbb{C}^{2}$ we introduce the $\mathrm{CR}$ equation [45]

$$
\overline{\mathfrak{M}} K \equiv \varlimsup_{\left(u_{\mathrm{B}}\right)} K+L \frac{\partial}{\partial u_{\mathrm{B}}} K=0
$$


and seek two independent (complex) solutions, $K_{1}=K_{1}\left(u_{\mathrm{B}}, \zeta, \bar{\zeta}\right), K_{2}=K_{2}\left(u_{\mathrm{B}}, \zeta, \bar{\zeta}\right)$ that define the embedding of $\mathfrak{I}^{+}$into $\mathbb{C}^{2}$ with coordinates $\left(K_{1}, K_{2}\right)$. We have immediately that $K_{1}=\bar{\zeta}=x-i y$ is a solution. The second solution is also easily found; we see directly from Eq. (4.13) [54],

$$
\mathrm{\partial}_{\left(u_{\mathrm{B}}\right)} T+L \dot{T}=0,
$$

that

$$
\tau=T\left(u_{\mathrm{B}}, \zeta, \bar{\zeta}\right),
$$

the inverse to $u_{\mathrm{B}}=X(\tau, \zeta, \bar{\zeta})$, is a $\mathrm{CR}$ function and that we can consider $\mathfrak{I}^{+}$to be embedded in the $\mathbb{C}^{2}$ of $(\tau, \bar{\zeta})$.

An important structure associated to any embeddable CR manifold of codimension one is its Levi form; this determines a metric on the CR structure as a bundle on the manifold [22]. As we have just discussed, $\mathfrak{I}^{+}$is just such a CR manifold, and one can show that its Levi form (in the CR structure induced by an asymptotically shear-free NGC) is proportional to the twist of the congruence. Hence, any CR structure on $\mathfrak{I}^{+}$which is generated by a congruence with its source on a real world line $\xi^{a}(s) \in \mathbb{M}$ is Levi-flat [7].

In the context of this review, the important observation is that the physical content of asymptotically shear-free NGCs is encoded in the corresponding CR structure. This gives a physical interpretation for $\mathrm{CR}$ structures in the setting of asymptotically flat spacetimes. It would be interesting for future research to study the relationship between our findings and those of [34], which demonstrates how the Einstein equations for algebraically special spacetimes can be realized in terms of the embeddable CR structures associated with their PNDs. 


\section{Tensorial Spin- $s$ Spherical Harmonics}

Some time ago, the generalization of ordinary spherical harmonics $Y_{l m}(\zeta, \bar{\zeta})$ to spin-weighted functions ${ }_{(s)} Y_{l m}(\zeta, \bar{\zeta})$ (e.g., $\left.[33,28,56]\right)$ was developed to allow for harmonic expansions of spin-weighted functions on the sphere. In this paper we have instead used the tensorial form of these spin-weighted harmonics, the tensorial spin-s spherical harmonics, which are formed by taking appropriate linear combinations of the ${ }_{(s)} Y_{l m}(\zeta, \bar{\zeta})[59]$ :

$$
Y_{l i \ldots k}^{s}=\sum K_{l i \ldots k(s)}^{s m} Y_{l m}
$$

where the indices obey $|s| \leq l$, and the number of spatial indices (i.e., $i \ldots k$ ) is equal to $l$. Explicitly, these tensorial spin-weighted harmonics can be constructed directly from the parametrized Lorentzian null tetrad, Eq. (1.1)-(1.2):

$$
\begin{aligned}
\hat{l}^{a} & =\frac{\sqrt{2}}{2(1+\zeta \bar{\zeta})}(1+\zeta \bar{\zeta}, \zeta+\bar{\zeta}, i \bar{\zeta}-i \zeta,-1+\zeta \bar{\zeta}), \\
\hat{n}^{a} & =\frac{\sqrt{2}}{2(1+\zeta \bar{\zeta})}(1+\zeta \bar{\zeta},-(\zeta+\bar{\zeta}), i \zeta-i \bar{\zeta}, 1-\zeta \bar{\zeta}), \\
\hat{m}^{a} & =\frac{\sqrt{2}}{2(1+\zeta \bar{\zeta})}\left(0,1-\bar{\zeta}^{2},-i\left(1+\bar{\zeta}^{2}\right), 2 \bar{\zeta}\right), \\
P & \equiv 1+\zeta \bar{\zeta}
\end{aligned}
$$

Taking the spatial parts of their duals, we obtain the one-forms

$$
\begin{aligned}
l_{i} & =\frac{-1}{\sqrt{2} P}(\zeta+\bar{\zeta},-i(\zeta-\bar{\zeta}),-1+\zeta \bar{\zeta}), \\
n_{i} & =\frac{1}{\sqrt{2} P}(\zeta+\bar{\zeta},-i(\zeta+\bar{\zeta}),-1+\zeta \bar{\zeta}), \\
m_{i} & =\frac{-1}{\sqrt{2} P}\left(1-\bar{\zeta}^{2},-i\left(1+\bar{\zeta}^{2}\right), 2 \bar{\zeta}\right), \\
c_{i} & =l_{i}-n_{i}=-\sqrt{2} i \epsilon_{i j k} m_{j} \bar{m}_{k} .
\end{aligned}
$$

From this we define $Y_{l i \ldots k}^{l}$ as [59]

$$
\begin{aligned}
& Y_{l i \ldots k}^{l}=m_{i} m_{j} \ldots m_{k}, \\
& Y_{l i \ldots k}^{-l}=\bar{m}_{i} \bar{m}_{j} \ldots \bar{m}_{k} .
\end{aligned}
$$

The other harmonics are determined by the action of the d-operator on the forms, Eq. (C.2), (with complex conjugates) via

$$
\begin{aligned}
ð l & =m, \\
ð m & =0, \\
ð n & =-m, \\
ð c & =2 m, \\
ð \bar{m} & =n-l=-c .
\end{aligned}
$$

Specifically, the spin- $s$ harmonics are defined by

$$
\begin{aligned}
Y_{l i \ldots k}^{s} & =\bar{\partial}^{l-s}\left(Y_{l i \ldots k}^{l}\right), \\
Y_{l i \ldots k}^{-|s|} & =\check{\partial}^{l-|s|}\left(Y_{l i \ldots k}^{-l}\right) .
\end{aligned}
$$


We now present a table of the tensorial spherical harmonics up to $l=2$, in terms of the tetrad. Higher harmonics can be found in [59].

$$
\begin{aligned}
& l=0 \\
& Y_{0}^{0}=1 \\
& \hline l=1 \\
& Y_{1 i}^{1}=m_{i}, \\
& Y_{1 i}^{0}=-c_{i}, \\
& Y_{1 i}^{-1}=\bar{m}_{i} \\
& \hline l=2 \\
& Y_{2 i j}^{2}=m_{i} m_{j}, \quad Y_{2 i j}^{1}=-\left(c_{i} m_{j}+m_{i} c_{j}\right), \quad Y_{2 i j}^{0}=3 c_{i} c_{j}-2 \delta_{i j} \\
& Y_{2 i j}^{-2}=\bar{m}_{i} \bar{m}_{j}, \quad Y_{2 i j}^{-1}=-\left(c_{i} \bar{m}_{j}+\bar{m}_{i} c_{j}\right) \quad
\end{aligned}
$$

In addition, it is useful to give the explicit relations between these different harmonics in terms of the $\circlearrowright$-operator and its conjugate. Indeed, we can see generally that applying $\partial$ once raises the spin index by one, and applying $\bar{\delta}$ lowers the index by one. This in turn means that

$$
\begin{aligned}
& \partial Y_{l i \ldots k}^{l}=0, \\
& \bar{\partial} Y_{l i \ldots k}^{-l}=0 .
\end{aligned}
$$

Other relations for $l \leq 2$ are given by

$$
\begin{aligned}
\bar{\partial} Y_{1 i}^{1} & =Y_{1 i}^{0}=ð Y_{1 i}^{-1}, \\
\partial Y_{1 i}^{0} & =-2 Y_{1 i}^{1}, \\
\bar{\partial} Y_{1 i}^{0} & =-2 Y_{1 i}^{-1}, \\
\bar{\partial} Y_{2 i j}^{2} & =Y_{2 i j}^{1}, \\
\bar{\partial}^{2} Y_{2 i j}^{2} & =Y_{2 i j}^{0}=\varlimsup^{2} Y_{2 i j}^{-2}, \\
\partial Y_{2 i j}^{0} & =-6 Y_{2 i j}^{1}, \\
\partial Y_{2 i j}^{1} & =-4 Y_{2 i j}^{2} .
\end{aligned}
$$

Finally, due to the nonlinearity of the theory, we have been forced throughout this review to consider products of the tensorial spin- $s$ spherical harmonics while expanding nonlinear expressions. These products can be expanded as a linear combination of individual harmonics using ClebschGordon expansions. The explicit expansions for products of harmonics with $l=1$ or $l=2$ are given below (we omit higher products due to the complexity of the expansion expressions). Further products can be found in [59, 42]. 


\section{C.1 Clebsch-Gordon expansions}

$$
\begin{aligned}
Y_{1 i}^{1} Y_{1 j}^{0} & =\frac{i}{\sqrt{2}} \epsilon_{i j k} Y_{1 k}^{1}+\frac{1}{2} Y_{2 i j}^{1}, \\
Y_{1 i}^{1} Y_{1 j}^{-1} & =\frac{1}{3} \delta_{i j}-\frac{i \sqrt{2}}{4} \epsilon_{i j k} Y_{1 k}^{0}-\frac{1}{12} Y_{2 i j}^{0}, \\
Y_{1 i}^{0} Y_{1 j}^{0} & =\frac{2}{3} \delta_{i j}+\frac{1}{3} Y_{2 i j}^{0}
\end{aligned}
$$

$$
\begin{aligned}
Y_{1 i}^{1} Y_{2 i j}^{2} & =Y_{3 i j k}^{3}, \\
Y_{1 i}^{0} Y_{2 j k}^{0} & =-\frac{4}{5} \delta_{k j} Y_{1 i}^{0}+\frac{6}{5}\left(\delta_{i j} Y_{1 k}^{0}+\delta_{i k} Y_{1 j}^{0}\right)+\frac{1}{5} Y_{3 i j k}^{0}, \\
Y_{1 i}^{1} Y_{2 j k}^{0} & =\frac{2}{5} Y_{1 i}^{1} \delta_{j k}-\frac{3}{5} Y_{1 j}^{1} \delta_{i k}-\frac{3}{5} Y_{1 k}^{1} \delta_{i j}+\frac{i}{\sqrt{2}}\left(\epsilon_{i k l} Y_{2 j l}^{1}+\epsilon_{i j l} Y_{2 k l}^{1}\right)+\frac{2}{5} Y_{3 i j k}^{1}, \\
Y_{1 i}^{1} Y_{2 j k}^{1} & =-\frac{1}{6} \partial\left(Y_{1 i}^{1} Y_{2 j k}^{0}\right), \\
Y_{2 i j}^{-1} Y_{1 k}^{1} & =\frac{3}{10} Y_{1 i}^{0} \delta_{j k}+\frac{3}{10} Y_{1 j}^{0} \delta_{i k}-\frac{1}{5} Y_{1 k}^{0} \delta_{i j}+\frac{i \sqrt{2}}{12}\left(\epsilon_{j k l} Y_{2 i l}^{0}+\epsilon_{i k l} Y_{2 l j}^{0}\right)-\frac{1}{30} Y_{3 i j k}^{0}, \\
Y_{1 i}^{0} Y_{2 j k}^{1} & =-\frac{2}{5} Y_{1 i}^{1} \delta_{j k}+\frac{3}{5} Y_{1 j}^{1} \delta_{i k}+\frac{3}{5} Y_{1 k}^{1} \delta_{i j}-\frac{i}{3 \sqrt{2}}\left(\epsilon_{i k l} Y_{2 j l}^{1}+\epsilon_{i j l} Y_{2 k l}^{1}\right)+\frac{4}{15} Y_{3 i j k}^{1}, \\
Y_{2 i j}^{2} Y_{1 k}^{-1} & =\frac{3}{10} Y_{1 i}^{0} \delta_{j k}+\frac{3}{10} Y_{1 j}^{0} \delta_{i k}-\frac{1}{5} Y_{1 k}^{0} \delta_{i j}-\frac{i \sqrt{2}}{12}\left(\epsilon_{j k l} Y_{2 i l}^{0}+\epsilon_{i k l} Y_{2 l j}^{0}\right)-\frac{1}{30} Y_{3 i j k}^{0}, \\
Y_{2 i j}^{2} Y_{1 k}^{0} & =\partial\left(Y_{2 i j}^{2} Y_{1 k}^{-1}\right)
\end{aligned}
$$

The Clebsch-Gordon expansions involving two $l=2$ harmonics have been used in the text. They are fairly long and are not given here but can be found in [59]. 


\section{D $\mathcal{H}$-Space Metric}

In the following, the derivation of the $\mathcal{H}$-space metric of $(4.24)$ is given. We begin with the cut function, $u_{\mathrm{B}}=Z\left(\xi^{a}(\tau), \zeta, \bar{\zeta}\right)=G(\tau, \zeta, \bar{\zeta})$ that satisfies the good-cut equation $\partial^{2} Z=\sigma^{0}(Z, \zeta, \bar{\zeta})$. The $(\zeta, \bar{\zeta})$ are (for the time being) completely independent of each other, though $\bar{\zeta}$ is to be treated as being 'close' the complex conjugate of $\zeta$. Taking the gradient of $Z\left(z^{a}, \zeta, \bar{\zeta}\right)$, multiplied by an arbitrary four vector $v^{a}$ (i.e., $V=v^{a} Z,_{a}$ ), we see that it satisfies the linear good cut equation,

$$
\begin{aligned}
\check{\partial}^{2} Z,_{a} & =\sigma^{0},{ }_{z} Z,_{a} \\
\check{\partial}^{2} V & =\sigma^{0},_{z} V .
\end{aligned}
$$

Let $V_{0}$ be a particular solution, and assume for the moment that the general solution can be written as

$$
Z,_{a}=V_{0} l_{a}^{*}
$$

with the four components of $l_{a}^{*}$ to be determined. Substituting Eq. (D.2) into the linearized goodcut equation, we have

$$
\begin{aligned}
& \check{\partial}^{2}\left(V_{0} l_{a}^{*}\right)=\sigma^{0},{ }_{z} V_{0} l_{a}^{*}, \\
& ð\left(l_{a}^{*} \mathrm{\partial}\left(V_{0}\right)+V_{0} \precsim l_{a}^{*}\right)=\sigma^{0},{ }_{z} V_{0} l_{a}^{*},
\end{aligned}
$$

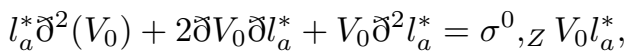

$$
\begin{aligned}
& 2 ð V_{0} \precsim l_{a}^{*}+V_{0} ð^{2} l_{a}^{*}=0, \\
& 2 V_{0} \partial V_{0} \partial l_{a}^{*}+V_{0}^{2} \check{\partial}^{2} l_{a}^{*}=0, \\
& \widetilde{\partial} V_{0}^{2} \check{\partial} l_{a}^{*}+V_{0}^{2} \check{\partial}^{2} l_{a}^{*}=0, \\
& ð\left(V_{0}^{2} \check{\partial} l_{a}^{*}\right)=0,
\end{aligned}
$$

which integrates immediately to

$$
V_{0}^{2} \precsim l_{a}^{*}=m_{a}^{*}
$$

where the $m_{a}^{*}$ are three independent $l=1, s=1$ functions.

By taking linear combinations they can be written as

$$
m_{a}^{*}=T_{a}^{b} \hat{m}_{b}=T_{a}^{b} \precsim \hat{l}_{b}
$$

where $\hat{l}_{a}$ is our usual $\hat{l}_{a}=\frac{\sqrt{2}}{2}\left(1,-\frac{\zeta+\bar{\zeta}}{1+\zeta \bar{\zeta}},-\frac{i(\bar{\zeta}-\zeta)}{1+\zeta \bar{\zeta}}, \frac{1-\zeta \bar{\zeta}}{1+\zeta \bar{\zeta}}\right)$, and the coefficients $T_{a}^{b}$ are functions only of the coordinates $z^{a}$. Assuming that the monopole term in $V^{2}$ is sufficiently large so that it has no zeros and then by rescaling $V$ we can write $V^{-2}$ as a monopole plus higher harmonics in the form

$$
V_{0}^{-2}=1+\partial W
$$

where $W$ is a spin-wt $s=-1$ quantity. From Eq. (D.3), we obtain

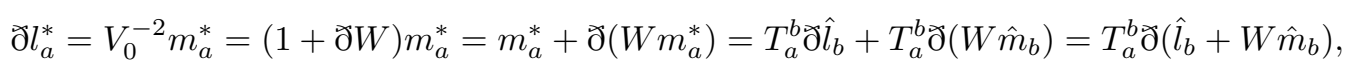

which integrates to

$$
l_{a}^{*}=T_{a}^{b}\left(\hat{l}_{a}+W \hat{m}_{a}\right) .
$$

The general solution to the linearized good-cut equation is thus

$$
\begin{aligned}
Z,_{a} & =V_{0} l_{a}^{*}=V_{0} T_{a}^{b}\left(\hat{l}_{b}+W \hat{m}_{b}\right), \\
V & =v^{a} Z,_{a}=V_{0} v^{a} T_{a}^{b}\left(\hat{l}_{b}+W \hat{m}_{b}\right) .
\end{aligned}
$$


We now demonstrate that

$$
\begin{aligned}
\left(g_{a b} v^{a} v^{b}\right)^{-1} & =(8 \pi)^{-1} \int V^{-2} d \Omega \\
d \Omega & =4 i \frac{d \zeta \wedge d \bar{\zeta}}{(1+\zeta \bar{\zeta})^{2}}
\end{aligned}
$$

In the integral of (D.6), we replace the independent variables $(\zeta, \bar{\zeta})$ by

$$
\zeta^{*}=\frac{\zeta+W}{1-W \bar{\zeta}}, \quad \bar{\zeta}^{*}=\bar{\zeta} .
$$

After some algebraic manipulation we obtain

$$
d \Omega^{*}=V_{0}^{-2} d \Omega
$$

and (surprisingly)

$$
\left(\hat{l}_{a}+W \hat{m}_{a}\right)=L_{a}^{*} \equiv \frac{\sqrt{2}}{2}\left(1,-\frac{\zeta^{*}+\bar{\zeta}}{1+\zeta^{*} \bar{\zeta}},-\frac{i\left(\bar{\zeta}-\zeta^{*}\right)}{1+\zeta^{*} \bar{\zeta}}, \frac{1-\zeta^{*} \bar{\zeta}}{1+\zeta^{*} \bar{\zeta}}\right)
$$

so that

$$
V=V_{0} v^{a} T_{a}^{b} L_{b}^{*}
$$

Inserting Eqs. (D.8), (D.9) and (D.11) into (D.6) we obtain

$$
\begin{aligned}
\left(g_{a b} v^{a} v^{b}\right)^{-1} & =(8 \pi)^{-1} \int\left(V_{0} v^{a} T_{a}^{b} L_{b}^{*}\right)^{-2} V_{0}^{2} d \Omega^{*} \\
& =(8 \pi)^{-1} \int\left(v^{a} T_{a}^{b} L_{b}^{*}\right)^{-2} d \Omega^{*} \\
& =(8 \pi)^{-1} \int\left(v^{* b} L_{b}^{*}\right)^{-2} d \Omega^{*}
\end{aligned}
$$

Using the form Eq. (D.10) the last integral can be easily evaluated (most easily done using $\theta$ and $\varphi$ ) leading to

$$
\begin{aligned}
\left(g_{a b} v^{a} v^{b}\right)^{-1} & =\left(\eta_{a b} v^{* a} v^{* b}\right)^{-1}=\left(T_{a}^{c} T_{b}^{d} \eta_{c d} v^{a} v^{b}\right)^{-1} \\
g_{a b} & =T_{a}^{c} T_{b}^{d} \eta_{c d}
\end{aligned}
$$

In particular, note that when $\sigma^{0}=0$ (i.e., the case of an everywhere shear-free NGC) $T_{b}^{a}=\delta_{b}^{a}$ and the metric on $\mathcal{H}$-space reduces to the complex Minkowski metric, as claimed throughout the text. We can go a step further by taking the derivative of Eq. (D.12) with respect to $v^{a}$ to find the covariant form of $v$, namely

$$
\frac{v_{a}}{\left(g_{a b} v^{a} v^{b}\right)^{2}}=\frac{g_{a b} v^{b}}{\left(g_{a b} v^{a} v^{b}\right)^{2}}=(8 \pi)^{-1} \int\left(v^{a} T_{a}^{b} L_{b}^{*}\right)^{-3} T_{a}^{b} L_{b}^{*} d \Omega^{*} .
$$




\section{E Shear-Free Congruences from Complex World Lines}

In this appendix, we show that the family of complex light-cones with apex on a complex world line in complex Minkowski space $\mathbb{M}_{\mathbb{C}}$ have null generators that form a real shear-free null geodesic congruence in real Minkowski space [7].

Theorem. There exists a mapping from the arbitrary complex-analytic world line $\xi^{a}(\tau) \in \mathbb{M}_{\mathbb{C}}$ to the real shear-free $N G C$ in $\mathbb{M}$ given by complex null displacements.

Proof: We first recall from Section 3 that regular real shear-free NGCs in $\mathbb{M}$ are parametrically given by

$$
x^{a}=u_{\mathrm{B}}\left(\hat{l}^{a}+\hat{n}^{a}\right)-L \overline{\hat{m}}^{a}-\bar{L} \hat{m}^{a}+\left(r^{*}-r_{0}\right) \hat{l}^{a}
$$

with

$$
\begin{aligned}
u_{\mathrm{B}} & =\xi^{b}(\tau) \widehat{l}_{b}, \quad \tau=T\left(u_{\mathrm{B}}, \zeta, \bar{\zeta}\right) \\
L\left(u_{\mathrm{B}}, \zeta, \bar{\zeta}\right) & =\xi^{a}(\tau) \hat{m}_{a}(\zeta, \bar{\zeta}), \\
\bar{L}\left(u_{\mathrm{B}}, \zeta, \bar{\zeta}\right) & =\bar{\xi}^{a}(\tau) \hat{\hat{m}}_{a}(\zeta, \bar{\zeta}) .
\end{aligned}
$$

The $\tau$ is taken so that $u_{\mathrm{B}}$ is real via Eq. (3.27): $\tau=s+i \Lambda(s, \zeta, \bar{\zeta})$, and $r_{0}$ is the arbitrary origin for the affine parameter along each geodesic of the congruence.

Beginning with the world line, $\xi^{a}(\tau)$, we add to it a specific complex null displacement (to be constructed)

$$
\begin{aligned}
L^{a} & =L_{0}^{a}\left(u_{\mathrm{B}}, \zeta, \bar{\zeta}\right)+r L_{1}^{a}\left(u_{\mathrm{B}}, \zeta, \bar{\zeta}\right), \\
L^{a} L_{a} & =L_{1}^{a} L_{1 a}=L_{0}^{a} L_{0 a}=0
\end{aligned}
$$

parametrized by the real variable $r$. We will show that the curve (complex null geodesic) given by

$$
x^{a}=\xi^{a}\left(T\left(u_{\mathrm{B}}, \zeta, \bar{\zeta}\right)\right)+L_{0}^{a}\left(u_{\mathrm{B}}, \zeta, \bar{\zeta}\right)+r L_{1}^{a}\left(u_{\mathrm{B}}, \zeta, \bar{\zeta}\right)
$$

with fixed $\left(u_{\mathrm{B}}, \zeta, \bar{\zeta}\right)$ but varying $r$, is identical to that given by Eq. (E.1).

This is demonstrated by taking the world line, $\xi^{a}(\tau)$, written in terms of its components $\left(\xi^{b} l_{b}, \xi^{b} n_{b}, \xi^{b} m_{b}, \xi^{b} \bar{m}_{b}\right)$ as

$$
\xi^{a}(\tau)=\xi^{b}(\tau) \hat{l}_{b} \hat{n}^{a}+\xi^{b}(\tau) \widehat{n}_{b} \hat{l}^{a}-\xi^{b}(\tau) \hat{m}_{b} \overline{\hat{m}}^{a}-\xi^{b}(\tau) \overline{\hat{m}}_{b} \hat{m}^{a}
$$

and replacing the $n^{a}$ by the identity

$$
\begin{aligned}
\hat{n}^{a} & =\sqrt{2} t^{a}-\hat{l}^{a} . \\
t^{a} & =\delta_{0}^{a} .
\end{aligned}
$$

This yields

$$
\xi^{a}(\tau)=\sqrt{2} \xi^{b}(\tau) \hat{l}_{b} t^{a}+\sqrt{2} \xi^{b}(\tau) t_{b} \hat{l}^{a}-2 \xi^{b}(\tau) \hat{l}_{b} \widehat{l}^{a}-\xi^{b}(\tau) \hat{m}_{b} \overline{\hat{m}}^{a}-\xi^{b}(\tau) \overline{\hat{m}}_{b} \hat{m}^{a} .
$$

Using the relations, Eq. (3.34), etc.,

$$
\begin{aligned}
u_{\mathrm{B}} & =\xi^{b}(\tau) \hat{l}_{b}, \quad \tau=T\left(u_{\mathrm{B}}, \zeta, \bar{\zeta}\right) \\
L\left(u_{\mathrm{B}}, \zeta, \bar{\zeta}\right) & =\xi^{b}(\tau) \hat{m}_{b} \\
\widetilde{L}\left(u_{\mathrm{B}}, \zeta, \bar{\zeta}\right) & =\xi^{a}(\tau) \overline{\hat{m}}_{a}(\zeta, \bar{\zeta})
\end{aligned}
$$


Eq. (E.4) becomes

$$
\xi^{a}(\tau)=\sqrt{2} u_{\mathrm{B}} t^{a}+\sqrt{2} \xi^{0}(\tau) \hat{l}^{a}-2 u_{\mathrm{B}} \hat{l}^{a}-L \overline{\hat{m}}^{a}-\tilde{L} \hat{m}^{a} .
$$

By adding the complex null vector (displacement),

$$
L^{a}=(\tilde{L}-\bar{L}) \hat{m}^{a}+\left(r-r_{0}+2 u_{\mathrm{B}}-\sqrt{2} \xi^{0}(\tau)\right) \hat{l}^{a}
$$

to both sides of Eq. (E.5), we obtain

$$
\begin{aligned}
& x^{a} \equiv \xi^{a}(\tau)+L^{a} \\
& x^{a}=\xi^{a}(\tau)+(\tilde{L}-\bar{L}) \hat{m}^{a}+\left(r-r_{0}+2 u_{\mathrm{B}}-\sqrt{2} \xi^{0}(\tau)\right) \hat{l}^{a} \\
& x^{a}=u_{\mathrm{B}} t^{a}-L \overline{\hat{m}}^{a}-\bar{L} \hat{m}^{a}+\left(r-r_{0}\right) \hat{l}^{a}
\end{aligned}
$$

To complete our task we now restrict the values of $\tau$ to those that produce a real $u$, namely

$$
\tau \rightarrow \tau^{(\mathrm{R})}=s+i \Lambda(s, \zeta, \bar{\zeta})
$$

and restrict $r$ to the real.

We see that by adding a null ray, combinations of $\hat{m}^{a}$ and $\hat{l}^{a}$, directly to the complex world line $\xi^{a}(\tau)$, we obtain a mapping of the complex world line directly to the real shear-free NGC, Eq. (E.1). Note that when the affine parameter, $r$, is chosen (complex) as $r=r_{0}-2 u+\sqrt{2} \xi^{0}(\tau)$ the $\hat{l}^{a}$ term drops out and we have the 'point' $\xi^{a}(\tau)$ surrounded by the embedded complex sphere, $z^{a}=\xi^{a}(\tau)+L_{0}^{a}(u, \zeta, \bar{\zeta})=\xi^{a}(\tau)+(\tilde{L}-\bar{L}) \hat{m}^{a}$. The ray can be thought of as having its origin on this surface.

We thus have the explicit relationship between the complex world line and the shear-free NGC, completing the proof.

\section{F The Generalized Good-Cut Equation}

Throughout this work, the Good-Cut Equation (GCEq) has played a major role in allowing us to study shear-free and asymptotically shear-free NGCs in asymptotically flat spacetimes. In this context, the GCEq is a partial differential equation on a topologically $S^{2}$ cut of $\mathfrak{I}^{+}$; due to the freedom in the choice of conformal factor on the two-sphere in the conformal compactification of asymptotically flat spacetimes, we can always take the space of null generators of $\mathfrak{I}^{+}$to be a metric two-spheres. However, one can imagine solving the GCEq on a surface which is only conformal to a metric two-sphere, we refer to such a PDE as the 'Generalized' GCEq, or $\mathrm{G}^{2} \mathrm{CEq}$ for short. In this appendix, we briefly motivate why one could be interested in the $\mathrm{G}^{2} \mathrm{CEq}$, and then prove that it can be reduced to the GCEq on the metric two-sphere by a coordinate transformation (this is essentially a proof of the conformal invariance of the GCEq) [6].

The study of horizons in the interior of spacetime is an important topic in a variety of areas, particularly quantum gravity. One interesting class of null horizons are the so-called 'vacuum non-expanding horizons', which are null 3-surfaces in a spacetime that have vanishing divergence and shear, and are topologically $\mathbb{R} \times S^{2}[13,14]$. In analogy with the setting on $\mathcal{I}^{+}$discussed in the body of this review, one can look for null geodesic congruences in the interior of a spacetime which have vanishing shear at their intersection with a vacuum non-expanding horizon. It has been shown that such 'horizon-shear-free' NGCs are described, where they 'cut' the horizon, by a good-cut equation on the topologically $S^{2}$ cut. Since we cannot freely rescale objects in the interior of the spacetime, this means that horizon-shear-free NGCs are described by the $\mathrm{G}^{2} \mathrm{CEq}$ [5]. 
Consider an arbitrary vacuum non-expanding horizon $\mathfrak{H}$ with associated $\mathrm{G}^{2} \mathrm{CEq}$. As in the asymptotic case, we consider the complexification $\mathfrak{H}_{\mathbb{C}}$ of the horizon when looking for solutions to the $\mathrm{G}^{2} \mathrm{CEq}$, and make use of local Bondi-like coordinates $(u, \zeta, \bar{\zeta})$. The $(\zeta, \bar{\zeta})$, which label the null generators of $\mathfrak{H}$, are the stereographic coordinates on the $S^{2}$ portion of $\mathfrak{H}\left(S^{2}\right.$ need not be a metric sphere); while the coordinate $u$ parametrizes the cross-sections of $\mathfrak{H}$. For $\mathfrak{H}_{\mathbb{C}}$, the $u$ is allowed to take complex values close to the real, while $\bar{\zeta}$ goes over to an independent variable close to the complex conjugate of $\zeta$. The context should make it clear when $\bar{\zeta}$ is actually the complex conjugate of $\zeta$. The distinction between the $\mathrm{GCEq}$ and the $\mathrm{G}^{2} \mathrm{CEq}$ is that the former lives on a 3 -surface $\mathfrak{H}$ whose $u=$ constant cross-sections are metric spheres, while for the latter equation the 2-surface metric is arbitrary.

As mentioned earlier, the 3 -surface $\mathfrak{H}$ is described by an $S^{2}$ worth of null geodesics with the cross sections given by $u=$ constant. The metric of the two-surface cross-sections are expressed in stereographic coordinates $(\zeta, \bar{\zeta})$ so that the metric takes the conformally flat form:

$$
d s^{2}=\frac{4 d \zeta d \bar{\zeta}}{P^{2}(\zeta, \bar{\zeta})},
$$

with $P(u, \zeta, \bar{\zeta})$ an arbitrary smooth nonvanishing function on the $(\zeta, \bar{\zeta})$-sphere, the extended complex plane (Riemann sphere). In the special case of a metric sphere we take

$$
P=P_{0} \equiv 1+\zeta \bar{\zeta}
$$

while in general we write

$$
P=V(u, \zeta, \bar{\zeta}) P_{0}
$$

The $\mathrm{G}^{2} \mathrm{CEq}$ contains the general $P$, while the special case using $P_{0}$ yields the GCEq.

For the most general situation, the $\mathrm{G}^{2} \mathrm{CEq}$ can be written as a differential equation for the function $u=G(\zeta, \bar{\zeta})$ :

$$
\overline{\bar{\partial}}^{2} G \equiv \partial_{\bar{\zeta}}\left(V^{2} P_{0}^{2} \partial_{\bar{\zeta}} G\right)=\bar{\sigma}(G, \zeta, \bar{\zeta})
$$

or

$$
P_{0}^{2} \partial_{\bar{\zeta}}^{2} G+2\left[P_{0}^{2} V^{-1} \partial_{\bar{\zeta}} V+P_{0} \zeta\right] \partial_{\bar{\zeta}} G=V^{-2} \bar{\sigma}(G, \zeta, \bar{\zeta}) .
$$

When $V=1$ we have the GCEq:

$$
\overline{\widetilde{\partial}}_{0}^{2} G \equiv \partial_{\bar{\zeta}}\left(P_{0}^{2} \partial_{\bar{\zeta}} G\right)=\bar{\sigma}(G, \zeta, \bar{\zeta})
$$

When the arbitrary spin-weight-2 function, $\bar{\sigma}(G, \zeta, \bar{\zeta})$ vanishes, we have the homogeneous $\mathrm{G}^{2} \mathrm{CEq}$ :

$$
\partial_{\bar{\zeta}}\left(P^{2} \partial_{\bar{\zeta}} G\right)=0
$$

It is now shown how, by a coordinate transformation of the (independent) complex stereographic coordinates $(\zeta, \bar{\zeta}), \mathrm{G}^{2} \mathrm{CEq}$ can be transformed into the GCEq. It must be remembered from our notation that $\bar{\zeta}^{*}$ (or $\bar{\zeta}$ ) is close to, but is not necessarily, the complex conjugate of $\zeta^{*}$ (or $\zeta$ ).

First rewrite the GCEq with stereographic coordinates $\left(\zeta^{*}, \bar{\zeta}^{*}\right)$ as

$$
\begin{aligned}
\bar{\partial}_{0 *}^{2} G & =\partial_{\bar{\zeta}^{*}}\left(P_{0}^{* 2} \partial_{\bar{\zeta}^{*}} G\right)=\bar{\sigma}^{*}\left(G, \zeta^{*}, \bar{\zeta}^{*}\right), \\
P_{0}^{*} & =1+\zeta^{*} \bar{\zeta}^{*},
\end{aligned}
$$

and the $\mathrm{G}^{2} \mathrm{CEq}$ as

$$
\overline{\bar{\partial}}^{2} G=\partial_{\bar{\zeta}}\left(V^{2} P_{0}^{2} \partial_{\bar{\zeta}} G\right)=\bar{\sigma}(G, \zeta, \bar{\zeta})
$$


We now apply the coordinate transformation

$$
\begin{aligned}
\bar{\zeta}^{*} & =\frac{\bar{\zeta}+W}{1-W \zeta} \equiv N(\zeta, \bar{\zeta}), \\
\zeta^{*} & =\zeta
\end{aligned}
$$

with $W$ (a spin-weight 1 function) defined from

$$
\begin{aligned}
V^{-2} & =1+\overline{\widetilde{ळ}}_{0} W=1+P_{0} \partial_{\bar{\zeta}} W-W \zeta \\
P_{0} & =1+\zeta \bar{\zeta}
\end{aligned}
$$

to Eq. (F.8). Substituting the derived relations,

$$
\begin{aligned}
P_{0}^{*} & =1+\zeta \bar{\zeta}^{*}=\frac{1+\zeta \bar{\zeta}}{1-W \zeta}=\frac{P_{0}}{1-W \zeta}, \\
\partial_{\bar{\zeta}} G & =\partial_{\bar{\zeta}^{*}} G \cdot \partial_{\bar{\zeta}} N \\
\partial_{\bar{\zeta}}^{2} G & =\partial_{\bar{\zeta}^{y}}^{2} G \cdot\left(\partial_{\bar{\zeta}} N\right)^{2}+\partial_{\bar{\zeta}^{*}} G \cdot \partial_{\bar{\zeta}}^{2} N \\
\partial_{\bar{\zeta}} N & =\frac{V^{-2}-W \zeta}{(1-W \zeta)^{2}}, \\
\partial_{\bar{\zeta}}^{2} N & =\frac{2 \zeta\left[V^{-2}-1\right] \partial_{\bar{\zeta}} W}{(1-W \zeta)^{3}}+\frac{\zeta \partial_{\bar{\zeta}} W}{(1-W \zeta)^{2}}-\frac{2 V^{-3} \partial_{\bar{\zeta}} V}{(1-W \zeta)^{2}},
\end{aligned}
$$

into Eq. (F.8), we have, after a bit of algebra,

$$
\begin{aligned}
\bar{\partial}_{0 *}^{2} G & =\partial_{\bar{\zeta}^{*}}\left(P_{0}^{* 2} \partial_{\bar{\zeta}^{*}} G\right) \\
& =F\left(\zeta^{*}, \bar{\zeta}^{*}\right) \sigma\left(G, \zeta\left(\zeta^{*}, \bar{\zeta}^{*}\right), \bar{\zeta}^{*}\left(\zeta^{*}, \bar{\zeta}^{*}\right)\right) \\
& \equiv \bar{\sigma}^{*}\left(G, \zeta^{*}, \bar{\zeta}^{*}\right),
\end{aligned}
$$

namely Eq. (F.6), the GCEq.

Hence, we see that the $\mathrm{G}^{2} \mathrm{CEq}$ is equivalent to the $\mathrm{GCEq}$ via the coordinate transformation (F.9). This means that the study of the $\mathrm{G}^{2} \mathrm{CEq}$ on a general 3-surface $\mathfrak{H}$ can be reduced to the study of the properties of the GCEq on a 3-surface whose cross-sections are metric spheres.

Remark 14. As in the main text, solutions to the $G C E q$ or $G^{2} C E q, u=G(\zeta, \bar{\zeta})$, known as 'goodcut functions', describe cross-sections of $\mathfrak{H}$ that are referred to as 'good cuts.' From the tangents to these good cuts, $L=\overline{\widetilde{\partial}} G$, one can construct null directions (pointing out of $\mathfrak{H}$ ) into the spacetime itself that determine a NGC whose shear vanishes at $\mathfrak{H}$. 


\section{References}

[1] Adamo, T.M., Bullimore, M., Mason, L. and Skinner, D., "Scattering amplitues and Wilson loops in twistor space", J. Phys. A: Math. Theor., 44, 454008, (2011). [DOI], [arXiv:1104.2890]. (Cited on page 74.)

[2] Adamo, T.M. and Newman, E.T., "The gravitational field of a radiating electromagnetic dipole", Class. Quantum Grav., 25, 245005, (2008). [DOI], [arXiv:0807.3537]. (Cited on page 63.)

[3] Adamo, T.M. and Newman, E.T., "Asymptotically stationary and static spacetimes and shear free null geodesic congruences", Class. Quantum Grav., 26, 155003, (2009). [DOI], [arXiv:0906.2409]. (Cited on pages 46 and 52.)

[4] Adamo, T.M. and Newman, E.T., "Electromagnetically induced gravitational perturbations", Class. Quantum Grav., 26, 015004, (2009). [DOI], [arXiv:0807.3671]. (Cited on pages 25 and 63.)

[5] Adamo, T.M. and Newman, E.T., "Vacuum non-expanding horizons and shear-free null geodesic congruences", Class. Quantum Grav., 26, 235012, (2009). [DOI], [arXiv:0908.0751]. (Cited on page 85.)

[6] Adamo, T.M. and Newman, E.T., "The Generalized Good Cut Equation", Class. Quantum Grav., 27, 245004, (2010). [DOI], [arXiv:1007.4215]. (Cited on pages 46, 75, and 85.)

[7] Adamo, T.M. and Newman, E.T., "The real meaning of complex Minkowski-space world-lines", Class. Quantum Grav., 27, 075009, (2010). [DOI], [arXiv:0911.4205]. (Cited on pages 9, 27, 30, 31, 73, 78, and 84.)

[8] Adamo, T.M. and Newman, E.T., "Light cones in relativity: Real, complex and virtual, with applications", Phys. Rev. D, 83, 044023, (2011). [DOI], [arXiv:1101.1052]. (Cited on pages 30, 31, 39, 60, and 70.)

[9] Aharony, O., Gubser, S.S., Maldacena, J.M., Ooguri, H. and Oz, Y., "Large $N$ field theories, string theory and gravity", Phys. Rep., 323, 183-386, (2000). [DOI], [hep-th/9905111]. (Cited on page 73.)

[10] Arcioni, G. and Dappiaggi, C., "Exploring the holographic principle in asymptotically flat spacetimes via the BMS group", Nucl. Phys. B, 674, 553-592, (2003). [DOI], [hep-th/0306142]. (Cited on page 73.$)$

[11] Arnowitt, R., Deser, S. and Misner, C.W., "Energy and the Criteria for Radiation in General Relativity", Phys. Rev., 118, 1100-1104, (1960). [DOI], [ADS]. (Cited on page 7.)

[12] Aronson, B. and Newman, E.T., "Coordinate systems associated with asymptotically shear-free null congruences", J. Math. Phys., 13, 1847-1851, (1972). [DOI]. (Cited on pages 28, 42, 49, and 69.)

[13] Ashtekar, A., Beetle, C. and Lewandowski, J., "Geometry of generic isolated horizons", Class. Quantum Grav., 19, 1195-1225, (2002). [DOI], [gr-qc/0111067]. (Cited on page 85.)

[14] Ashtekar, A. and Krishnan, B., "Isolated and Dynamical Horizons and Their Applications", Living Rev. Relativity, 7, lrr-2004-10, (2004). URL (accessed 28 April 2011): http://www.livingreviews.org/lrr-2004-10. (Cited on page 85.)

[15] Bergmann, P.G., "Non-Linear Field Theories", Phys. Rev., 75, 680-685, (1949). [DOI], [ADS]. (Cited on page 72.$)$

[16] Bondi, H., van der Burg, M.G.J. and Metzner, A.W.K., "Gravitational Waves in General Relativity. VII. Waves from Axi-Symmetric Isolated Systems", Proc. R. Soc. London, Ser. A, 269, 21-52, (1962). [DOI], [ADS]. (Cited on pages 7, 10, 17, and 24.)

[17] Bousso, R., "The holographic principle", Rev. Mod. Phys., 74, 825-874, (2002). [DOI], [hepth/0203101]. (Cited on page 73.) 
[18] Bramson, B.D., "Relativistic Angular Momentum for Asymptotically Flat Einstein-Maxwell Manifolds", Proc. R. Soc. London, Ser. A, 341, 463-490, (1975). [DOI]. (Cited on page 74.)

[19] Bramson, B., "Do electromagnetic waves harbour gravitational waves?", Proc. R. Soc. London, Ser. $A, \mathbf{4 6 2}, 1987-2000,(2006)$. [DOI]. (Cited on page 63.)

[20] Chruściel, P.T. and Friedrich, H., eds., The Einstein Equations and the Large Scale Behavior of Gravitational Fields: 50 Years of the Cauchy Problem in General Relativity, (Birkhäuser, Basel; Boston, 2004). [Google Books]. (Cited on page 72.)

[21] Corvino, J. and Schoen, R.M., "On the asymptotics for the vacuum Einstein constraint equations", J. Differ. Geom., 73, 185-217, (2006). [gr-qc/0301071]. (Cited on page 72.)

[22] Dragomir, S. and Tomassini, G., Differential Geometry and Analysis on CR Manifolds, (Birkhäuser, Boston; Basel; Berlin, 2006). [Google Books]. (Cited on pages 77 and 78.)

[23] Frauendiener, J., "Conformal Infinity", Living Rev. Relativity, 7, lrr-2004-1, (2004). URL (accessed 31 July 2009):

http://www.livingreviews.org/lrr-2004-1. (Cited on pages 8 and 17.)

[24] Friedrich, H., "On the Existence of $n$-Geodesically Complete or Future Complete Solutions of Einstein's Field Equations with Smooth Asymptotic Structure", Commun. Math. Phys., 107, 587-609, (1986). [DOI]. (Cited on page 72.)

[25] Frittelli, S., Kozameh, C.N., Newman, E.T., Rovelli, C. and Tate, R.S., "Fuzzy spacetime from a null-surface version of general relativity", Class. Quantum Grav., 14, A143-A154, (1997). [DOI], [gr-qc/9603061]. (Cited on page 72.)

[26] Frittelli, S. and Newman, E.T., "Pseudo-Minkowskian coordinates in asymptotically flat space-times", Phys. Rev. D, 55, 1971-1976, (1997). [DOI], [ADS]. (Cited on page 64.)

[27] Gel'fand, I.M., Graev, M.I. and Vilenkin, N.Y., Generalized Functions, Vol. 5: Integral geometry and representation theory, (Academic Press, New York; London, 1966). (Cited on pages 64 and 65.)

[28] Goldberg, J.N., Macfarlane, A.J., Newman, E.T., Rohrlich, F. and Sudarshan, E.C.G., "Spin- $s$ Spherical Harmonics and ð”", J. Math. Phys., 8, 2155-2161, (1967). [DOI]. (Cited on pages 19 and 79.)

[29] Goldberg, J.N. and Sachs, R.K., "A Theorem on Petrov Types", Acta Phys. Pol., 22, 13-23, (1962). Republished as DOI:10.1007/s10714-008-0722-5. (Cited on pages 9, 24, and 26.)

[30] Hallidy, W. and Ludvigsen, M., "Momentum and Angular Momentum in the $H$-Space of Asymptotically Flat, Einstein-Maxwell Space-Times", Gen. Relativ. Gravit., 10, 7-30, (1979). [DOI]. (Cited on page 74.)

[31] Hansen, R.O. and Newman, E.T., "A complex Minkowski space approach to twistors", Gen. Relativ. Gravit., 6, 361-385, (1975). [DOI]. (Cited on pages 41 and 43.)

[32] Hansen, R.O., Newman, E.T., Penrose, R. and Tod, K.P., "The Metric and Curvature Properties of $\mathcal{H}$-Space", Proc. R. Soc. London, Ser. A, 363, 445-468, (1978). [DOI], [ADS]. (Cited on pages 43, 44, and 74.)

[33] Held, A., Newman, E.T. and Posadas, R., "The Lorentz Group and the Sphere", J. Math. Phys., 11, 3145-3154, (1970). [DOI]. (Cited on pages 64, 65, 67, and 79.)

[34] Hill, C. D., Lewandowski, J. and Nurowski, P, "Einstein's equations and the embedding of 3dimensional CR manifolds", Indiana Univ. Math. J., 57, 3131-3176, (2008). [DOI], [arXiv:0709.3660]. (Cited on page 78.) 
[35] Hugget, S.A. and Tod, K.P., An Introduction to Twistor Theory, London Mathematical Society Student Texts, 4, (Cambridge University Press, Cambridge; New York, 1994), 2nd edition. [Google Books]. (Cited on page 75.)

[36] Ivancovich, J., Kozameh, C.N. and Newman, E.T., "Green's functions of the edh operators", J. Math. Phys., 30, 45-52, (1989). [DOI]. (Cited on page 43.)

[37] Ko, M., Newman, E.T. and Tod, K.P., "H-H-Space and Null Infinity", in Esposito, F.P. and Witten, L., eds., Asymptotic Structure of Space-Time, Proceedings of a Symposium on Asymptotic Structure of Space-Time (SOASST), held at the University of Cincinnati, Ohio, June 14-18, 1976, pp. 227-271, (Plenum Press, New York, 1977). (Cited on page 9.)

[38] Kozameh, C.N. and Newman, E.T., "Electromagnetic dipole radiation fields, shear-free congruences and complex centre of charge world lines", Class. Quantum Grav., 22, 4667-4678, (2005). [DOI], [gr-qc/0504093]. (Cited on page 34.)

[39] Kozameh, C.N. and Newman, E.T., "The large footprints of H-space on asymptotically flat spacetimes", Class. Quantum Grav., 22, 4659-4665, (2005). [DOI], [gr-qc/0504022]. (Cited on pages 8, 28, 29, 42, and 69.)

[40] Kozameh, C.N., Newman, E.T., Santiago-Santiago, J.G. and Silva-Ortigoza, G., "The universal cut function and type II metrics", Class. Quantum Grav., 24, 1955-1979, (2007). [DOI], [gr-qc/0612004]. (Cited on pages 7, 8, 27, 28, 29, 35, and 54.)

[41] Kozameh, C.N., Newman, E.T. and Silva-Ortigoza, G., "On the physical meaning of the RobinsonTrautman-Maxwell fields", Class. Quantum Grav., 23, 6599-6620, (2006). [DOI], [gr-qc/0607074]. (Cited on pages 7, 44, and 51.)

[42] Kozameh, C.N., Newman, E.T. and Silva-Ortigoza, G., "On extracting physical content from asymptotically flat spacetime metrics", Class. Quantum Grav., 25, 145001, (2008). [DOI], [arXiv:0802.3314]. (Cited on pages $7,8,25,67,70$, and 80.)

[43] Landau, L.D. and Lifshitz, E.M., The classical theory of fields, (Pergamon Press; Addison-Wesley, Oxford; Reading, MA, 1962), 2nd edition. (Cited on pages 13, 35, 60, 61, 64, and 67.)

[44] Lewandowski, J. and Nurowski, P., "Algebraically special twisting gravitational fields and CR structures", Class. Quantum Grav., 7, 309-328, (1990). [DOI]. (Cited on page 77.)

[45] Lewandowski, J., Nurowski, P. and Tafel, J., "Einstein's equations and realizability of CR manifolds", Class. Quantum Grav., 7, L241-L246, (1990). [DOI]. (Cited on page 77.)

[46] Lind, R.W., "Shear-free, twisting Einstein-Maxwell metrics in the Newman-Penrose formalism", Gen. Relativ. Gravit., 5, 25-47, (1974). [DOI]. (Cited on page 51.)

[47] Maldacena, J.M., "The Large- $N$ Limit of Superconformal Field Theories and Supergravity", Adv. Theor. Math. Phys., 2, 231-252, (1998). [DOI], [hep-th/9711200]. (Cited on page 73.)

[48] Mason, L. J. and Skinner, D., "Gravity, Twistors and the MHV Formalism", Commun. Math. Phys., 294, 827-862, (2010). [DOI], [arXiv:0808.3907]. (Cited on page 74.)

[49] Newman, E.T., "Heaven and Its Properties", Gen. Relativ. Gravit., 7, 107-111, (1976). [DOI]. (Cited on pages 9 and 44.)

[50] Newman, E.T., "Maxwell fields and shear-free null geodesic congruences", Class. Quantum Grav., 21, 3197-3221, (2004). [DOI]. (Cited on pages 34, 36, 37, and 40.)

[51] Newman, E.T., "Asymptotic twistor theory and the Kerr theorem", Class. Quantum Grav., 23, 33853392, (2006). [DOI], [gr-qc/0512079]. (Cited on pages 75 and 76.) 
[52] Newman, E.T., "Newton's second law, radiation reaction and type II Einstein-Maxwell fields", Class. Quantum Grav., 28, 245003, (2011). [DOI], [arXiv:1109.4106]. (Cited on page 52.)

[53] Newman, E.T., Couch, E., Chinnapared, K., Exton, A., Prakash, A. and Torrence, R., "Metric of a Rotating, Charged Mass", J. Math. Phys., 6, 918-919, (1965). [DOI]. (Cited on pages 9, 49, and 69.)

[54] Newman, E.T. and Nurowski, P., "CR structures and asymptotically flat spacetimes", Class. Quantum Grav., 23, 3123-3127, (2006). [DOI], [gr-qc/0511119]. (Cited on page 78.)

[55] Newman, E.T. and Penrose, R., "An Approach to Gravitational Radiation by a Method of Spin Coefficients", J. Math. Phys., 3, 566-578, (1962). [DOI], [ADS]. (Cited on pages 10, 21, and 22.)

[56] Newman, E.T. and Penrose, R., "Note on the Bondi-Metzner-Sachs Group", J. Math. Phys., 7, 863-870, (1966). [DOI], [ADS]. (Cited on pages 10 and 79.)

[57] Newman, E.T. and Penrose, R., "Spin-coefficient formalism", Scholarpedia, 4(6), 7445, (2009). URL (accessed 30 July 2009):

http://www.scholarpedia.org/article/Spin-coefficient_formalism. (Cited on page 21.)

[58] Newman, E.T. and Posadas, R., "Motion and Structure of Singularities in General Relativity", Phys. Rev., 187, 1784-1791, (1969). [DOI], [ADS]. (Cited on page 51.)

[59] Newman, E.T. and Silva-Ortigoza, G., "Tensorial spin-s harmonics", Class. Quantum Grav., 23, 497-509, (2006). [DOI], [gr-qc/0508028]. (Cited on pages 10, 11, 79, 80, and 81.)

[60] Newman, E.T. and Tod, K.P., "Asymptotically flat space-times", in Held, A., ed., General Relativity and Gravitation: One Hundred Years After the Birth of Albert Einstein, 2, pp. 1-36, (Plenum Press, New York, 1980). (Cited on pages 17, 18, 19, 21, 52, and 65.)

[61] Newman, E.T. and Unti, T.W.J., "Behavior of Asymptotically Flat Empty Spaces", J. Math. Phys., 3, 891-901, (1962). [DOI], [ADS]. (Cited on page 22.)

[62] Penrose, R., "Asymptotic Properties of Fields and Space-Times", Phys. Rev. Lett., 10, 66-68, (1963). [DOI], [ADS]. (Cited on pages 8 and 17.)

[63] Penrose, R., "Zero Rest-Mass Fields Including Gravitation: Asymptotic Behaviour", Proc. R. Soc. London, Ser. A, 284, 159-203, (1965). [DOI], [ADS]. (Cited on pages 8 and 17.)

[64] Penrose, R., "Twistor Algebra", J. Math. Phys., 8, 345-366, (1967). [DOI]. (Cited on page 75.)

[65] Penrose, R., "Relativistic symmetry groups", in Barut, A.O., ed., Group Theory in Non-Linear Problems, Proceedings of the NATO Advanced Study Institute, held in Istanbul, Turkey, August 7-18, 1972, NATO ASI Series C, 7, pp. 1-58, (Reidel, Dordrecht; Boston, 1974). (Cited on pages 10 and 25.)

[66] Penrose, R. and Rindler, W., Spinors and space-time, Vol. 1: Two-spinor calculus and relativistic fields, Cambridge Monographs on Mathematical Physics, (Cambridge University Press, Cambridge; New York, 1984). [Google Books]. (Cited on pages 11, 21, 22, 25, and 26.)

[67] Penrose, R. and Rindler, W., Spinors and space-time, Vol. 2: Spinor and twistor methods in spacetime geometry, Cambridge Monographs on Mathematical Physics, (Cambridge University Press, Cambridge; New York, 1986). [Google Books]. (Cited on pages 29, 75, and 76.)

[68] Petrov, A.Z., "The Classification of Spaces Defining Gravitational Fields", Gen. Relativ. Gravit., 32, 1665-1685, (2000). [DOI]. (Cited on page 26.)

[69] Pirani, F.A.E., "Invariant Formulation of Gravitational Radiation Theory", Phys. Rev., 105(3), 10891099, (1957). [DOI]. (Cited on page 26.) 
[70] Robinson, I., "Null Electromagnetic Fields", J. Math. Phys., 2, 290-291, (1961). [DOI]. (Cited on page 7.)

[71] Robinson, I. and Trautman, A., "Some spherical gravitational waves in general relativity", Proc. $R$. Soc. London, Ser. A, 265, 463-473, (1962). [DOI]. (Cited on pages 44 and 50.)

[72] Sachs, R.K., "Gravitational Waves in General Relativity. VIII. Waves in Asymptotically Flat SpaceTime", Proc. R. Soc. London, Ser. A, 270, 103-126, (1962). [DOI], [ADS]. (Cited on pages 7, 10, and 20.)

[73] Sachs, R.K., "Gravitational radiation", in DeWitt, C.M. and DeWitt, B., eds., Relativity, Groups and Topology, Lectures delivered at Les Houches during the 1963 session of the Summer School of Theoretical Physics, University of Grenoble, pp. 523-562, (Gordon and Breach, New York, 1964). (Cited on page 24.)

[74] Sommers, P., "The geometry of the gravitational field at spacelike infinity", J. Math. Phys., 19, 549-554, (1978). [DOI], [ADS]. (Cited on page 7.)

[75] Szabados, L.B., "Quasi-Local Energy-Momentum and Angular Momentum in General Relativity", Living Rev. Relativity, 12, lrr-2009-4, (2009). URL (accessed 31 July 2009):

http://www.livingreviews.org//rr-2009-4. (Cited on pages 25, 48, and 49.)

[76] 't Hooft, G., "A Planar Diagram Theory for Strong Interactions", Nucl. Phys. B, 72, 461, (1974). [DOI]. (Cited on page 73.)

[77] 't Hooft, G., "Dimensional reduction in quantum gravity", in Ali, A., Ellis, J. and Randjbar-Daemi, S., eds., Salamfestschrift, A Collection of Talks from the Conference on Highlights of Particle and Condensed Matter Physics, ICTP, Trieste, Italy, 8-12 March 1993, World Scientific Series in 20th Century Physics, 4, (World Scientific, Singapore; River Edge, NJ, 1994). [arXiv:gr-qc/9310026]. (Cited on page 73.)

[78] Witten, E., "Anti-de Sitter space and holography", Adv. Theor. Math. Phys., 2, 253-291, (1998). [hep-th/9802150]. (Cited on page 73.) 